\title{
Stellar laboratories
}

\section{New Zr IV-VII, Xe IV-V, and Xe VII oscillator strengths and the AI, Zr, and $X e$ abundances in the hot white dwarfs G191-B2B and

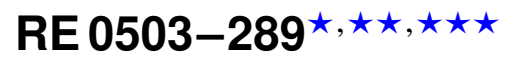

\author{
T. Rauch ${ }^{1}$, S. Gamrath ${ }^{2}$, P. Quinet ${ }^{2,3}$, L. Löbling ${ }^{1}$, D. Hoyer ${ }^{1}$, K. Werner ${ }^{1}$, J. W. Kruk ${ }^{4}$, and M. Demleitner ${ }^{5}$ \\ 1 Institute for Astronomy and Astrophysics, Kepler Center for Astro and Particle Physics, Eberhard Karls University, Sand 1, \\ 72076 Tübingen, Germany \\ e-mail: rauch@astro.uni-tuebingen.de \\ 2 Physique Atomique et Astrophysique, Université de Mons - UMONS, 7000 Mons, Belgium \\ 3 IPNAS, Université de Liège, Sart Tilman, 4000 Liège, Belgium \\ ${ }^{4}$ NASA Goddard Space Flight Center, Greenbelt, MD 20771, USA \\ 5 Astronomisches Rechen-Institut (ARI), Centre for Astronomy of Heidelberg University, Mönchhofstraße 12-14, 69120 Heidelberg, \\ Germany
}

Received 27 September 2016 / Accepted 6 November 2016

\begin{abstract}
Context. For the spectral analysis of high-resolution and high-signal-to-noise spectra of hot stars, state-of-the-art non-local thermodynamic equilibrium (NLTE) model atmospheres are mandatory. These are strongly dependent on the reliability of the atomic data that is used for their calculation.

Aims. To search for zirconium and xenon lines in the ultraviolet (UV) spectra of G191-B2B and RE 0503-289, new Zr IV-VII, Xe IV$\mathrm{V}$, and Xe VII oscillator strengths were calculated. This allows, for the first time, determination of the $\mathrm{Zr}$ abundance in white dwarf (WD) stars and improvement of the Xe abundance determinations.

Methods. We calculated Zr IV-VII, Xe IV-V, and Xe VII oscillator strengths to consider radiative and collisional bound-bound transitions of $\mathrm{Zr}$ and $\mathrm{Xe}$ in our NLTE stellar-atmosphere models for the analysis of their lines exhibited in UV observations of the hot WDs G191-B2B and RE 0503-289.

Results. We identified one new ZrIV, 14 new Zrv, and ten new Zr VI lines in the spectrum of RE 0503-289. Zr was detected for the first time in a WD. We measured a $\mathrm{Zr}$ abundance of $-3.5 \pm 0.2$ (logarithmic mass fraction, approx. 11500 times solar). We identified five new Xe VI lines and determined a Xe abundance of $-3.9 \pm 0.2$ (approx. 7500 times solar). We determined a preliminary photospheric Al abundance of $-4.3 \pm 0.2$ (solar) in RE 0503-289. In the spectra of G191-B2B, no Zr line was identified. The strongest Zr IV line (1598.948 $\AA$ ) in our model gave an upper limit of $-5.6 \pm 0.3$ (approx. 100 times solar). No Xe line was identified in the UV spectrum of G191-B2B and we confirmed the previously determined upper limit of $-6.8 \pm 0.3$ (ten times solar).

Conclusions. Precise measurements and calculations of atomic data are a prerequisite for advanced NLTE stellar-atmosphere modeling. Observed Zr IV-VI and Xe VI-VII line profiles in the UV spectrum of RE 0503-289 were simultaneously well reproduced with our newly calculated oscillator strengths.
\end{abstract}

Key words. atomic data - line: identification - stars: abundances - stars: individual: G191-B2B - stars: individual: RE0503-289 virtual observatory tools

\section{Introduction}

The DO-type white dwarf (WD) star RE 0503-289 (WD 0501+527, McCook \& Sion 1999a,b), exhibits many lines of the trans-iron elements $\mathrm{Zn}$ (atomic number $Z=30$ ), Ga (31), Ge (32), As (33), Se (34), Kr (36), Mo (42), Sn (50), Te (52), I (53), Xe (54), and Ba (56) in its ultraviolet spectrum. These were initially identified by Werner et al. (2012b), who

\footnotetext{
^ Based on observations with the NASA/ESA Hubble Space Telescope, obtained at the Space Telescope Science Institute, which is operated by the Association of Universities for Research in Astronomy, Inc., under NASA contract NAS5-26666.

$\star \star$ Based on observations made with the NASA-CNES-CSA Far Ultraviolet Spectroscopic Explorer.

$\star \star \star$ Tables A.9-A.12 and B.5-B.7 are only available via the German Astrophysical Virtual Observatory (GAVO) service TOSS (http:// dc.g-vo.org/TOSS).
}

determined the $\mathrm{Kr}$ and $\mathrm{Xe}$ abundances (Sect. 8) based on atomic data available at that time. Calculations of transition probabilities for $\mathrm{Zn}, \mathrm{Ga}, \mathrm{Ge}, \mathrm{Kr}, \mathrm{Mo}, \mathrm{Xe}$, and $\mathrm{Ba}$ in the subsequent years allowed precise abundance measurements for these elements (Rauch et al. 2014a, 2015b, 2012, 2016a, 2014b, 2015a, 2016b, respectively).

Here we report that we have identified lines of an additional element, namely zirconium (40) which has never been detected before in WDs, and calculated new ZrIV-VII transition probabilities to determine its photospheric abundance. To verify the Xe abundance determination of Werner et al. (2012b), we calculated much more complete Xe IV-V and Xe VI transition probabilities.

The hot, hydrogen-rich, DA-type WD G191-B2B (WD 0501+527, McCook \& Sion 1999a,b) is a primary flux reference standard for all absolute calibrations from 1000 to 
Table 1. Column densities (in $\mathrm{cm}^{-2}$ ) and radial velocities (in $\mathrm{km} \mathrm{s}^{-1}$ ) used to model interstellar clouds in the line of sight toward RE 0503-289.

\begin{tabular}{|c|c|c|c|}
\hline \multicolumn{2}{|c|}{ Mg II $\lambda 2796.35 \AA$} & \multicolumn{2}{|c|}{$\operatorname{Mg}$ II $\lambda 2803.53 \AA$} \\
\hline$N$ & $v_{\text {rad }}$ & $N$ & $v_{\text {rad }}$ \\
\hline $2.9 \times 10^{12}$ & +15.0 & $4.5 \times 10^{12}$ & +15.0 \\
\hline $2.6 \times 10^{12}$ & +7.0 & $3.8 \times 10^{12}$ & +7.0 \\
\hline $8.0 \times 10^{11}$ & -0.5 & $1.2 \times 10^{12}$ & -0.5 \\
\hline $4.6 \times 10^{11}$ & -4.5 & $8.5 \times 10^{11}$ & -5.5 \\
\hline $4.5 \times 10^{11}$ & -26.5 & $5.0 \times 10^{11}$ & -29.5 \\
\hline $7.3 \times 10^{11}$ & -43.5 & $1.0 \times 10^{12}$ & -38.5 \\
\hline
\end{tabular}

$25000 \AA$ (Bohlin 2007). Rauch et al. (2013) presented a detailed spectral analysis of this star. Based on their model, Rauch et al. (2014a, 2015b, 2014b) identified $\mathrm{Zn}, \mathrm{Ga}$, and $\mathrm{Ba}$ lines in the observed UV spectrum and determined the abundances of these elements.

We briefly introduce our observational data in Sect. 2. The discovery of the interstellar Mg II $\lambda \lambda 2796.35,2803.53 \AA$ resonance doublet and its modelling is shown in Sect. 3. Our model atmospheres are described in Sect. 4. We start our spectral analysis with a search for $\mathrm{Al}$ lines and an abundance determination in Sect. 5. The $\mathrm{Zr}$ transition-probability calculation, line identification, and abundance analysis are presented in Sect. 6, followed by the same for Xe in Sect.7. We summarize our results and conclude in Sect. 8.

\section{Observations}

For RE 0503-289, we analyzed ultraviolet (UV) observations that were obtained with the Far Ultraviolet Spectroscopic Explorer (FUSE, $910 \AA<\lambda<1188 \AA$, resolving power $R=$ $\lambda / \Delta \lambda \approx 20000$ ) and the Hubble Space Telescope/Space Telescope Imaging Spectrograph (HST/STIS, $1144 \AA<\lambda<3073 \AA$, $R \approx 45800$ ). These were described in detail by Werner et al. (2012b) and Rauch et al. (2016b), respectively.

For G191-B2B, we used the FUSE observation described by Rauch et al. (2013) and the high-dispersion échelle spectrum (HST/STIS, $1145-3145 \AA, R \approx 100000$, Rauch et al. 2013) available from the CALSPEC ${ }^{1}$ database.

To compare observations with synthetic spectra, the latter were convolved with Gaussians to model the respective resolving power. The observed spectra are shifted to rest wavelengths according to radial-velocity measurements of $v_{\text {rad }}=24.56 \mathrm{~km} \mathrm{~s}^{-1}$ (Lemoine et al. 2002) and $25.8 \mathrm{~km} \mathrm{~s}^{-1}$ for G191-B2B and RE 0503-289 (our value), respectively.

\section{Interstellar line absorption}

Rauch et al. (2016b) found that the interstellar line absorption toward RE0503-289 has a multi-velocity structure (radial-velocities $-40 \mathrm{~km} \mathrm{~s}^{-1}<v_{\mathrm{rad}}<+18 \mathrm{~km} \mathrm{~s}^{-1}$ ). In the HST/STIS spectra of RE0503-289, the interstellar Mg II $\lambda \lambda 2796.35,2803.53 \AA$ resonance lines $\left(3 \mathrm{~s}^{2} \mathrm{~S}_{1 / 2}-3 \mathrm{p}^{2} \mathrm{P}_{3 / 2}^{\mathrm{o}}\right.$ and $3 \mathrm{~s}^{2} \mathrm{~S}_{1 / 2}-3 \mathrm{p}^{2} \mathrm{P}_{1 / 2}^{\mathrm{o}}$ with oscillator strengths of 0.608 and 0.303, respectively) are prominent (Fig. 1) and corroborate such a structure. Table 1 displays the parameters that were used to fit the observation.

\footnotetext{
1 http://www. stsci.edu/hst/observatory/cdbs/calspec. html
}

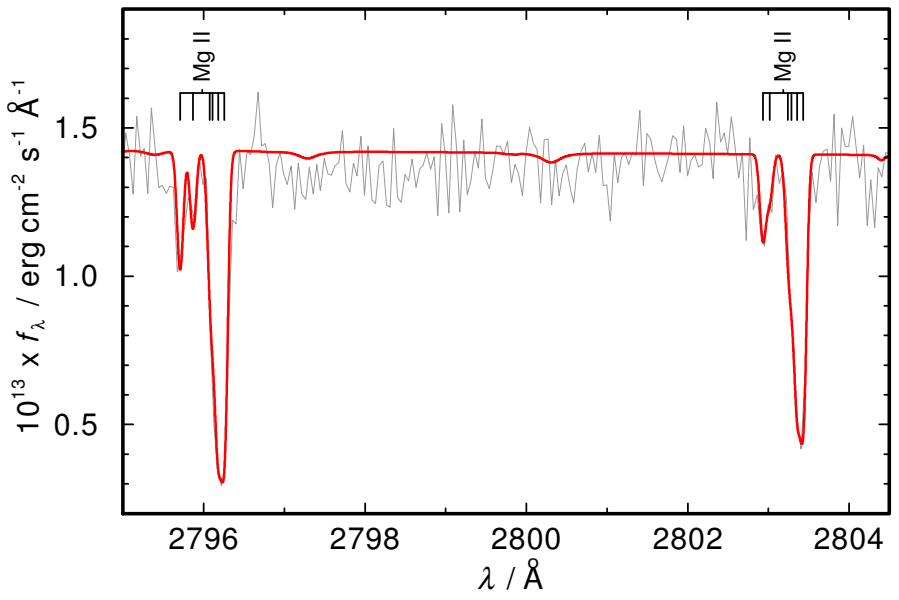

Fig. 1. Section of the STIS spectrum of RE0503-289 with the interstellar Mg II $\lambda \lambda 2796.35,2803.53 \AA$ lines.

\section{Model atmospheres and atomic data}

We calculated plane-parallel, chemically homogeneous modelatmospheres in hydrostatic and radiative equilibrium with the Tübingen non-local thermodynamic equilibrium (NLTE) Model Atmosphere Package (TMAP ${ }^{2}$, Werner et al. 2003, 2012a). Model atoms were retrieved from the Tübingen Model Atom Database (TMAD 3 , Rauch \& Deetjen 2003) that has been constructed as part of the Tübingen contribution to the German Astrophysical Virtual Observatory $\left(\mathrm{GAVO}^{4}\right)$.

The effective temperatures, surface gravities, and photospheric abundances of G191-B2B ( $T_{\text {eff }}=60000 \pm 2000 \mathrm{~K}$, $\log \left(\mathrm{g} / \mathrm{cm} \mathrm{s}^{-2}\right)=7.6 \pm 0.05$, Rauch et al. 2013) and RE 0503-289 $\quad\left(T_{\text {eff }}=70000 \pm 2000 \mathrm{~K}, \quad \log g=7.50 \pm 0.1\right.$, Rauch et al. 2016b) were previously analyzed with TMAP models. We adopt these parameters for our calculations.

ZrIV-VII and XeIV-VII were represented by the $\mathrm{Zr}$ and Xe model atoms with so-called super levels and super lines that were calculated with a statistical approach via our Iron Opacity and Interface ( $\mathrm{IrOnIc}^{5}$, Rauch \& Deetjen 2003; Müller-Ringat 2013). To enable IrOnIc to read our new $\mathrm{Zr}$ and $\mathrm{Xe}$ data, we transferred it into Kurucz-formatted files (cf., Rauch et al. $2015 b$ ). The statistics of our $\mathrm{Zr}$ and $\mathrm{Xe}$ model atoms is listed in Table 2.

For $\mathrm{Zr}$ and $\mathrm{Xe}$ and all other species, level dissolution (pressure ionization) following Hummer \& Mihalas (1988) and Hubeny et al. (1994) is accounted for. Broadening for all Al, Zr, and Xe lines due to the quadratic Stark effect is calculated using approximate formulae given by Cowley $(1970,1971)$.

All spectral energy distributions (SEDs) that were calculated for this analysis are available via the registered Theoretical Stellar Spectra Access (TheoSSA ${ }^{6}$ ) GAVO service.

\section{Aluminum in RE 0503-289}

The Al abundance in RE 0503-289 was hitherto undetermined. TMAD provides a recently extended Al model atom (Table 3). We used it to search for Al lines in the UV and optical spectra of G191-B2B and RE0503-289, especially for Al IV lines,

\footnotetext{
2 http://astro.uni-tuebingen.de/ TMAP

3 http://astro.uni-tuebingen.de/ TMAD

4 http://www.g-vo.org

5 http://astro.uni-tuebingen.de/ TIRO

6 http://dc.g-vo.org/theossa
} 
Table 2. Statistics of Zr IV-VII and Xe IV-V, VII atomic levels and line transitions from Tables A.9-A.12 and B.5-B.7, respectively.

\begin{tabular}{lcccc}
\hline \hline Ion & Atomic levels & Lines & Super levels & Super lines \\
\hline Zr IV & 52 & 135 & 7 & 20 \\
Zr v & 135 & 1449 & 7 & 22 \\
Zr VI & 96 & 1098 & 7 & 12 \\
Zr VII & 83 & 947 & 7 & 15 \\
\hline Total & 366 & 3629 & 28 & 69 \\
\hline Xe IV & 94 & 1391 & 7 & 16 \\
Xe V & 65 & 616 & 7 & 15 \\
Xe VI & 90 & 243 & 7 & 16 \\
Xe VII & 60 & 491 & 7 & 19 \\
\hline Total & 309 & 2741 & 28 & 66 \\
\hline
\end{tabular}

Notes. Xe VI is shown for completeness. ${ }^{(a)}$ Atomic level and line data taken from Gallardo et al. (2015).

Table 3. Statistics of the Al model atom used in our calculations compared to our previous analyses (e.g., Rauch et al. 2013, 2016b).

\begin{tabular}{lccccc}
\hline \hline & \multicolumn{2}{c}{ This work } & & \multicolumn{2}{c}{ Previous analyses } \\
\cline { 2 - 3 } \cline { 5 - 6 } Ion & Atomic levels & Lines & & Atomic levels & Lines \\
\hline Al II & & & & 1 & 0 \\
Al III & 24 & 70 & & 7 & 10 \\
Al IV & 61 & 276 & & 6 & 3 \\
Al V & 43 & 168 & & 6 & 4 \\
Al VI & 1 & 0 & & 1 & 0 \\
\hline & 129 & 514 & & 21 & 17 \\
\hline
\end{tabular}

because, in both stars, this is the dominant ionization stage in the line-forming region $(-4 \lesssim \log m \lesssim 0.5$, Figs. 2,3$)$. So far, only Al III lines were identified in the UV spectrum of G191-B2B, namely $\lambda \lambda 1854.714,1862.787 \AA$ (Holberg et al. 1998) and $\lambda \lambda 1379.668,1384.130,1605.764,1611.812,1611.854 \AA$

(Rauch et al. 2013, logarithmic mass fraction of $\mathrm{Al}=$ $-4.95 \pm 0.2$ ).

The only additional $\mathrm{Al}$ lines found in the observed spectra of G191-B2B are Al III $\lambda \lambda$ 1935.840, 1935.863, and 1935.949 $\AA$ (Fig. 4). AlIV lines in our model are entirely too weak to detect them in the observations. Compared to the available STIS spectrum of G191-B2B, that of RE 0503-289 has a much lower signal-to-noise ratio $(\mathrm{S} / \mathrm{N})$ that hampers detection of $\mathrm{Al}$ lines. Al III $\lambda \lambda 1384.130 \AA$ is the only line that is present in the observation and is well reproduced at a solar $\mathrm{Al}$ abundance (-4.28 \pm 0.2$)$. This result is based on a single line only, and thus it must be judged as uncertain. It is, however, at least an upper abundance limit. The derived abundance is, nonetheless, in good agreement with the expectation (interpolation in Fig. 10). To improve the Al abundance measurement, better UV spectra for RE 0503-289 are highly desirable.

\section{Zirconium}

\subsection{Oscillator-strength calculations for Zr IV-VII ions}

Radiative decay rates (oscillator strengths and transition probabilities) were computed using the pseudo-relativistic HartreeFock (HFR) method originally introduced by Cowan (1981), and modified for taking into account core-polarization effects

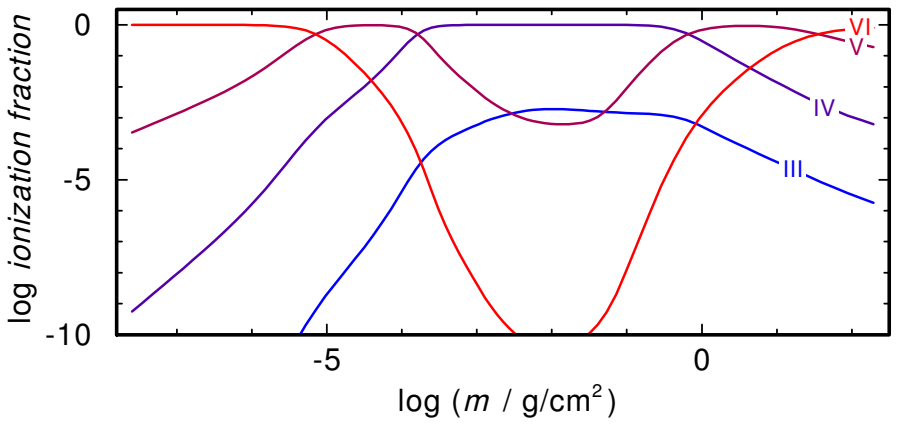

Fig. 2. Al ionization fractions in our G191-B2B model. $m$ is the column mass, measured from the outer boundary of our model atmospheres.

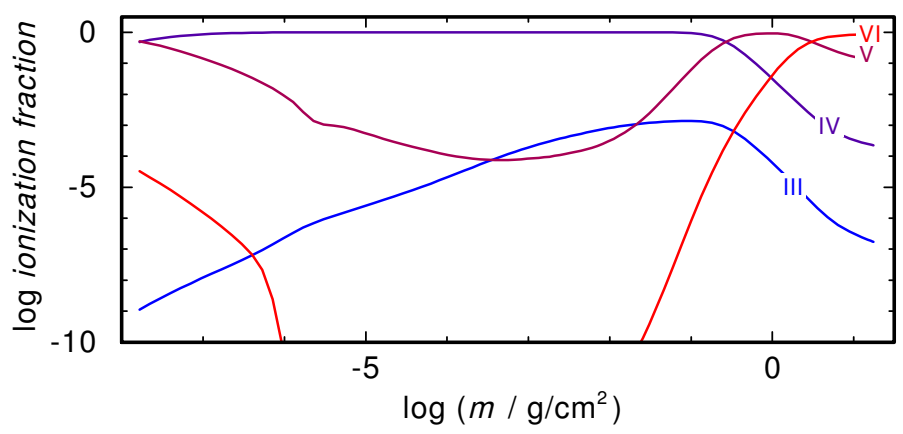

Fig. 3. As Fig. 2, for RE 0503-289.

(CPOL), giving rise to the HFR+CPOL approach (e.g., Quinet et al. 1999, 2002).

For Zr IV, configuration interaction was considered among the configurations $4 \mathrm{~s}^{2} 4 \mathrm{p}^{6} n \mathrm{~d}(n=4-9), 4 \mathrm{~s}^{2} 4 \mathrm{p}^{6} n \mathrm{~s} \quad(n=5-$ $9), 4 s^{2} 4 p^{6} n g(n=5-9), 4 s^{2} 4 p^{6} n i \quad(n=7-9), 4 s^{2} 4 p^{5} 4 d 5 p$, $4 \mathrm{~s}^{2} 4 \mathrm{p}^{5} 4 \mathrm{~d} 4 \mathrm{f}$, and $4 \mathrm{~s}^{2} 4 \mathrm{p}^{5} 4 \mathrm{~d} 5 \mathrm{f}$ for the even parity, and $4 \mathrm{~s}^{2} 4 \mathrm{p}^{6} n \mathrm{p}$ $(n=5-9), 4 \mathrm{~s}^{2} 4 \mathrm{p}^{6} n \mathrm{f}(n=4-9), 4 \mathrm{~s}^{2} 4 \mathrm{p}^{6} n \mathrm{~h}(n=6-9), 4 \mathrm{~s}^{2} 4 \mathrm{p}^{6} n \mathrm{k}$ $(n=8-9), 4 s^{2} 4 p^{5} 4 d^{2}, 4 s^{2} 4 p^{5} 4 d 5 s$, and $4 s^{2} 4 p^{5} 4 d 5 d$ for the odd parity. The core-polarization parameters were the dipole polarizability of a Zr VI ionic core as reported by Fraga et al. (1976), that is, $\alpha_{\mathrm{d}}=2.50$ a.u., and the cut-off radius corresponding to the HFR mean value $\langle r\rangle$ of the outermost core orbital (4p), that is, $r_{\mathrm{c}}=1.34 \mathrm{a}$.u. Using the experimental energy levels taken from the analysis by Reader \& Acquista (1997), the average energies and spin-orbit parameters of $4 \mathrm{~s}^{2} 4 \mathrm{p}^{6} n \mathrm{~d}(n=4-6), 4 \mathrm{~s}^{2} 4 \mathrm{p}^{6} n \mathrm{~s}$ $(n=5-8), 4 \mathrm{~s}^{2} 4 \mathrm{p}^{6} n \mathrm{~g}(n=5-9), 4 \mathrm{~s}^{2} 4 \mathrm{p}^{6} n \mathrm{p}(n=5-7), 4 \mathrm{~s}^{2} 4 \mathrm{p}^{6} n \mathrm{f}$ $(n=4-6)$, and $4 s^{2} 4 p^{6} 6 h$ configurations were adjusted using a well-established least-squares fitting procedure in which the mean deviations with experimental data were found to be equal to $0 \mathrm{~cm}^{-1}$ for the even parity and $6 \mathrm{~cm}^{-1}$ for the odd parity.

For $\mathrm{ZrV}$, the configurations explicitly included in the HFR model were $4 \mathrm{~s}^{2} 4 \mathrm{p}^{6}, 4 \mathrm{~s}^{2} 4 \mathrm{p}^{5} n \mathrm{p}(n=5-7), 4 \mathrm{~s}^{2} 4 \mathrm{p}^{5} n \mathrm{f}(n=4-7)$, $4 \mathrm{~s}^{4} \mathrm{p}^{6} n \mathrm{~d}(n=4-7), 4 \mathrm{~s} 4 \mathrm{p}^{6} n \mathrm{~s}(n=5-7), 4 \mathrm{~s}^{2} 4 \mathrm{p}^{4} 4 \mathrm{~d}^{2}, 4 \mathrm{~s}^{2} 4 \mathrm{p}^{4} 4 \mathrm{~d} 5 \mathrm{~s}$, and $4 \mathrm{~s}^{2} 4 \mathrm{p}^{4} 5 \mathrm{~s}^{2}$ for the even parity, and $4 \mathrm{~s}^{2} 4 \mathrm{p}^{5} n \mathrm{~d}(n=4-7)$, $4 \mathrm{~s}^{2} 4 \mathrm{p}^{5} n \mathrm{~s}(n=5-10), 4 \mathrm{~s}^{2} 4 \mathrm{p}^{5} n \mathrm{~g}(n=5-7), 4 \mathrm{~s}^{4} \mathrm{p}^{6} n \mathrm{p}(n=5-$ $7), 4 s^{4} p^{6} n f(n=4-7), 4 s^{2} 4 p^{4} 4 d 5 p$, and $4 s^{2} 4 p^{4} 4 d 4 f$ for the odd parity. Core-polarization effects were estimated using $\alpha_{\mathrm{d}}=$ 0.08 a.u. and $r_{\mathrm{c}}=0.45$ a.u. These values correspond to a Nilike Zr XIII ionic core, with $3 \mathrm{~d}$ as an outermost core subshell. In this ion, the semi-empirical process was performed to optimize the average energies, spin-orbit parameters, and electrostatic interaction. Slater integrals corresponding to $4 \mathrm{p}^{6}, 4 \mathrm{p}^{5} n \mathrm{p}$ $(n=5-6), 4 \mathrm{p}^{5} 4 \mathrm{f}, 4 \mathrm{~s} 4 \mathrm{p}^{6} 4 \mathrm{~d}, 4 \mathrm{p}^{5} n \mathrm{~d}(n=4-7), 4 \mathrm{p}^{5} n \mathrm{~s}(n=5-$ $10), 4 p^{5} n g(n=5-6)$, and $4 s^{4} p^{6} 5 p$ configurations using the experimental levels reported by Reader \& Acquista (1979) and 


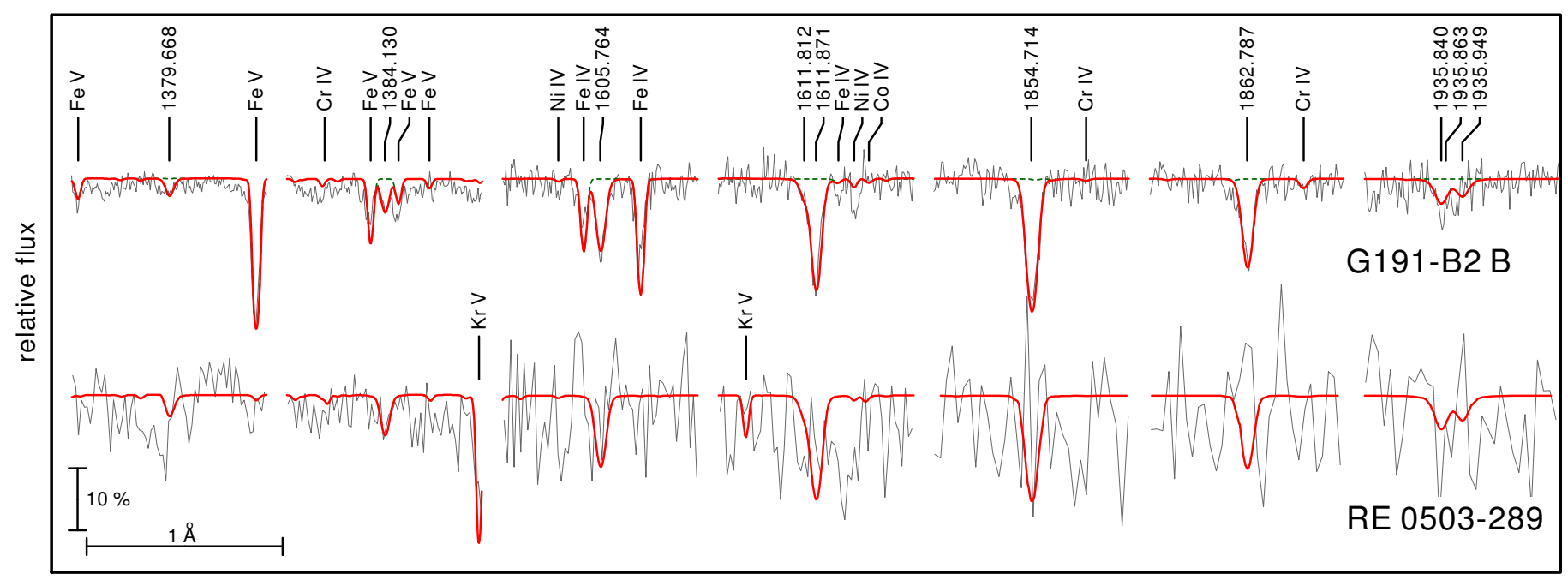

$\Delta \lambda / \AA$

Fig. 4. Comparison of sections of the STIS spectra with our models for G191-B2B (top) and RE 0503-289 (bottom). The Al abundances are $1.1 \times 10^{-5}\left(0.2\right.$ times the solar value, Rauch et al. 2013) and $5.3 \times 10^{-5}$ (solar), respectively. In the top part, the green dashed line is a spectrum calculated without Al. Prominent lines are marked, the identified Al III lines with their wavelengths.

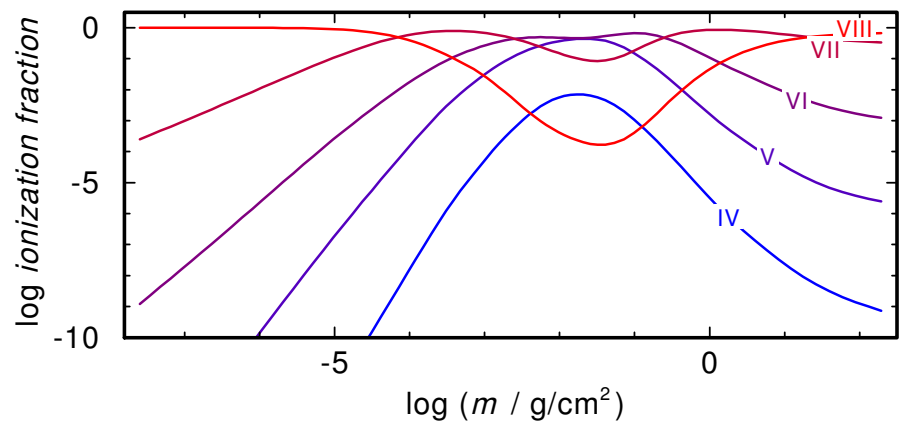

Fig. 5. Like Fig. 2, for Zr.

Khan et al. (1981). The mean deviations between calculated and experimental energies were $77 \mathrm{~cm}^{-1}$ and $91 \mathrm{~cm}^{-1}$ for even and odd parities, respectively.

In the case of Zr VI, the HFR method was used with the interacting configurations $4 \mathrm{~s}^{2} 4 \mathrm{p}^{5}, 4 \mathrm{~s}^{2} 4 \mathrm{p}^{4} n \mathrm{p}(n=5-6), 4 \mathrm{~s}^{2} 4 \mathrm{p}^{4} n \mathrm{f}$ $(n=4-6), 4 \mathrm{~s}^{4} \mathrm{p}^{5} n \mathrm{~d}(n=4-6), 4 \mathrm{~s}^{5} \mathrm{p}^{5} n \mathrm{~s}(n=5-6), 4 \mathrm{p}^{6} n \mathrm{p}(n=5-$ 6), $4 \mathrm{p}^{6} n \mathrm{f}(n=4-6), 4 \mathrm{~s}^{2} 4 \mathrm{p}^{3} 4 \mathrm{~d}^{2}, 4 \mathrm{~s}^{2} 4 \mathrm{p}^{3} 4 \mathrm{~d} 5 \mathrm{~s}$, and $4 \mathrm{~s}^{2} 4 \mathrm{p}^{3} 5 \mathrm{~s}^{2}$ for the odd parity, and $4 \mathrm{~s} 4 \mathrm{p}^{6}, 4 \mathrm{~s}^{2} 4 \mathrm{p}^{4} n \mathrm{~d}(n=4-6), 4 \mathrm{~s}^{2} 4 \mathrm{p}^{4} n \mathrm{~s}(n=5-$ $6), 4 \mathrm{~s}^{2} 4 \mathrm{p}^{4} n \mathrm{~g}(n=5-6), 4 \mathrm{~s} 4 \mathrm{p}^{5} n \mathrm{p}(n=5-6), 4 \mathrm{~s} 4 \mathrm{p}^{5} n \mathrm{f}(n=4-6)$, $4 \mathrm{p}^{6} n \mathrm{~s}(n=5-6), 4 \mathrm{p}^{6} n \mathrm{~d}(n=4-6), 4 \mathrm{~s}^{2} 4 \mathrm{p}^{3} 4 \mathrm{~d} 5 \mathrm{p}$, and $4 \mathrm{~s}^{2} 4 \mathrm{p}^{3} 4 \mathrm{~d} 4 \mathrm{f}$ for the even parity. Core-polarization effects were estimated using the same $\alpha_{\mathrm{d}}$ and $r_{\mathrm{c}}$ values as those considered in $\mathrm{Zr} \mathrm{V}$. The radial integrals corresponding to $4 p^{5}, 4 p^{4} 5 p, 4 s 4 p^{6}, 4 p^{4} 5 d, 4 p^{4} 5 s$, and $4 p^{4} 6 s$ were adjusted to minimize the differences between the calculated Hamiltonian eigenvalues and the experimental energy levels taken from Reader \& Lindsay (2016). In this process, we found mean deviations equal to $111 \mathrm{~cm}^{-1}$ in the odd parity and $221 \mathrm{~cm}^{-1}$ in the even parity.

Finally, for Zr VII, the configurations included in the HFR model were $4 \mathrm{~s}^{2} 4 \mathrm{p}^{4}, 4 \mathrm{~s}^{2} 4 \mathrm{p}^{3} n \mathrm{p}(n=5-6), 4 \mathrm{~s}^{2} 4 \mathrm{p}^{3} n \mathrm{f}(n=4-$ $6), 4 \mathrm{~s}^{4} \mathrm{p}^{4} n \mathrm{~d}(n=4-6), 4 \mathrm{~s}^{4} \mathrm{p}^{4} n \mathrm{~s}(n=5-6), 4 \mathrm{p}^{5} n \mathrm{p}(n=5-$ 6), $4 \mathrm{p}^{5} n \mathrm{f}(n=4-6), 4 \mathrm{~s}^{2} 4 \mathrm{p}^{2} 4 \mathrm{~d}^{2}, 4 \mathrm{~s}^{2} 4 \mathrm{p}^{2} 4 \mathrm{~d} 5 \mathrm{~s}$, and $4 \mathrm{~s}^{2} 4 \mathrm{p}^{2} 5 \mathrm{~s}^{2}$ for the even parity, and $4 \mathrm{~s}^{4} \mathrm{p}^{5}, 4 \mathrm{~s}^{2} 4 \mathrm{p}^{3} n \mathrm{~d}(n=4-6), 4 \mathrm{~s}^{2} 4 \mathrm{p}^{3} n \mathrm{~s}$ $(n=5-6), 4 \mathrm{~s}^{2} 4 \mathrm{p}^{3} n \mathrm{~g}(n=5-6), 4 \mathrm{~s} 4 \mathrm{p}^{4} n \mathrm{p}(n=5-6), 4 \mathrm{~s} 4 \mathrm{p}^{4} n \mathrm{f}$ $(n=4-6), 4 \mathrm{p}^{5} n \mathrm{~s}(n=5-6), 4 \mathrm{p}^{5} n \mathrm{~d}(n=4-6), 4 \mathrm{~s}^{2} 4 \mathrm{p}^{2} 4 \mathrm{~d} 5 \mathrm{p}$, and $4 s^{2} 4 p^{2} 4 d 4 f$ for the odd parity. The same core-polarization

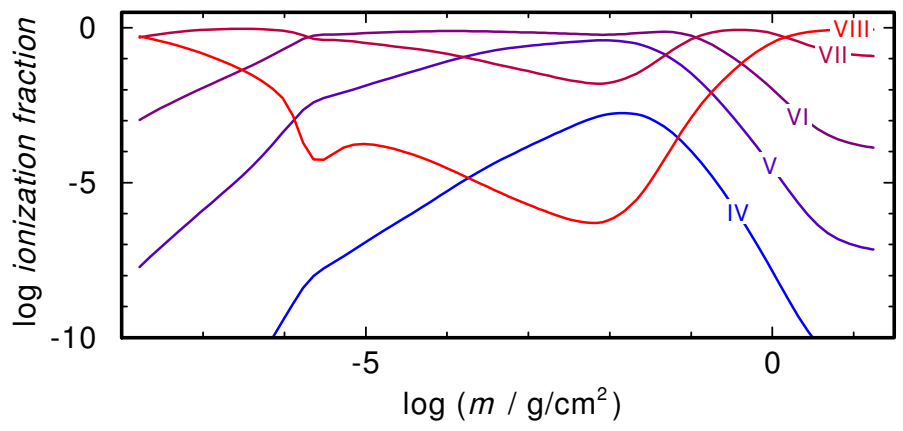

Fig. 6. Like Fig. 3, for Zr.

parameters as those used in $\mathrm{Zr} \mathrm{V}$ and $\mathrm{Zr}$ VI calculations were considered while the radial integrals of $4 p^{4}, 4 p^{3} 5 p, 4 s 4 p^{5}, 4 p^{3} 4 d$, and $4 p^{3} 5 \mathrm{~s}$ were optimized with the experimental energy levels taken from Reader \& Acquista (1976), Rahimullah et al. (1978), Khan et al. (1983). Although having established level values, the $4 \mathrm{p}^{3} 4 \mathrm{f}$ configuration was not fitted because it appeared very strongly mixed with experimentally unknown configurations such as $4 s 4 p^{4} 4 d$, and $4 s^{2} 4 p^{2} 4 d^{2}$ according to our HFR calculations. This semi-empirical process led to mean deviations of $695 \mathrm{~cm}^{-1}$ and $479 \mathrm{~cm}^{-1}$ for even and odd parities, respectively.

The parameters adopted in our computations are summarized in Tables A.1-A.4 while computed and available experimental energies are compared in Tables A.5-A.8, for Zr IV-VII, respectively. Tables A.9-A.12 give the HFR weighted oscillator strengths $(\log g f)$ and transition probabilities $\left(g A\right.$, in $\left.\mathrm{s}^{-1}\right)$ together with the numerical values $\left(\right.$ in $\mathrm{cm}^{-1}$ ) of the lower and upper energy levels and the corresponding wavelengths (in $\AA$ ). In the last column of each table, we also give the cancellation factor, $C F$, as defined by Cowan (1981). We note that very low values of this factor (typically <0.05) indicate strong cancellation effects in the calculation of line strengths. In these cases, the corresponding $g f$ and $g A$ values could be very inaccurate and therefore need to be considered with some care. However, very few of the transitions appearing in Tables A.9-A.12 are affected. 
T. Rauch et al.: Stellar laboratories. VIII.

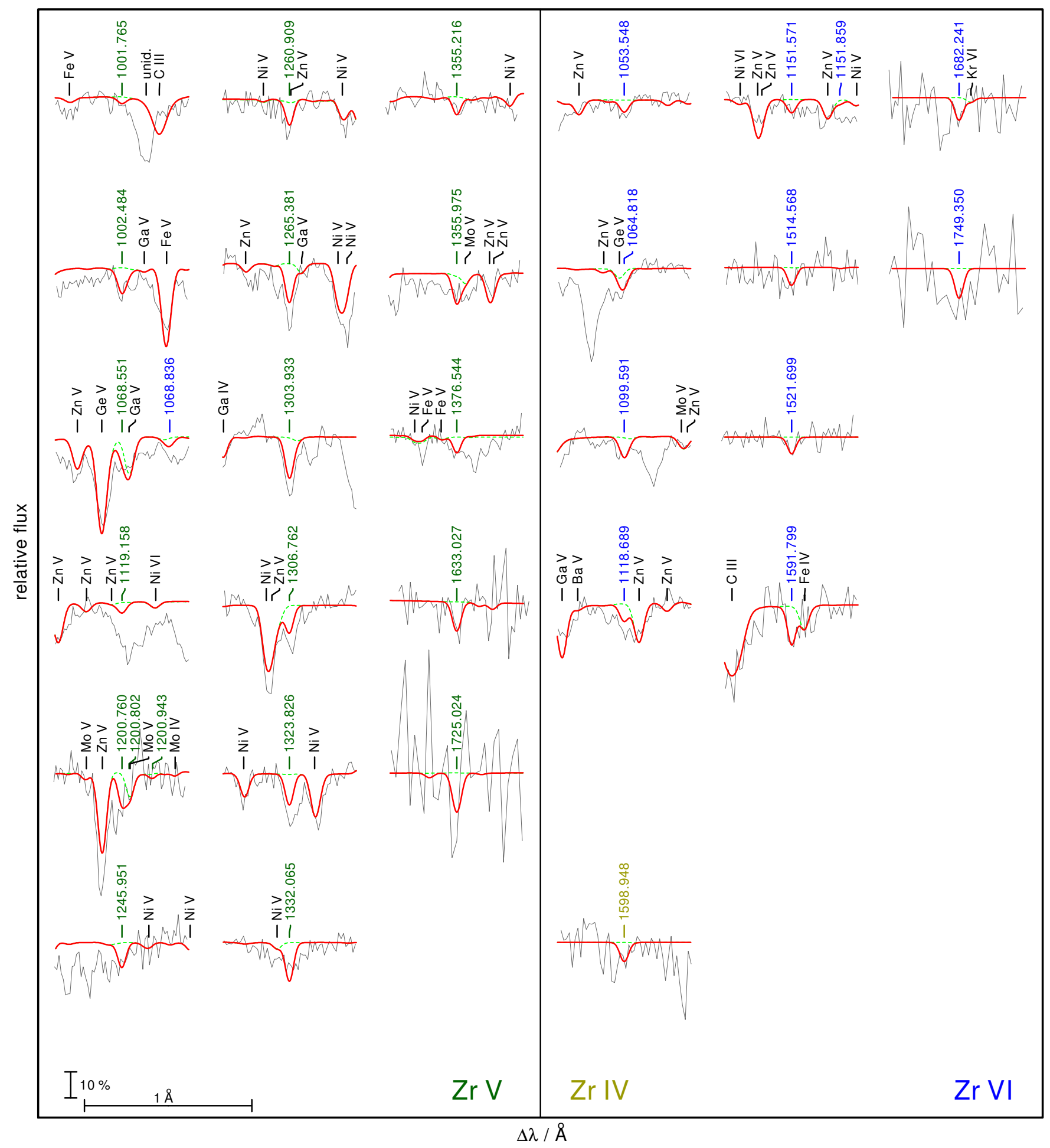

Fig. 7. Identified Zr IV (bottom of right panel), Zr V (left panel), and Zr VI (right panel) lines in the FUSE $(\lambda<1188 \AA$ ) and HST/STIS observations of RE 0503-289. The model (thick, red line) was calculated with an abundance of $\log \mathrm{Zr}=-3.5$. The dashed green spectrum was calculated without $\mathrm{Zr}$. Prominent lines are marked, the $\mathrm{Zr}$ lines with their wavelengths from Tables A.9-A.11.

These tables are provided via the registered GAVO Tübingen Oscillator Strengths Service $\left(\operatorname{TOSS}^{7}\right)$.

\subsection{Zr line identification and abundance analysis}

In the FUSE and HST/STIS observations of RE0503-289, we identified ZrIV-VI lines (Table 4). The observation is well

\footnotetext{
7 http://dc.g-vo.org/TOSS
}

reproduced by our model calculated with a mass fraction of $\log \mathrm{Zr}=-3.5 \pm 0.2$ (Fig. 7). The $\mathrm{Zr}$ IV/V/VI ionization equilibria are matched by our model.

In our synthetic spectra for G191-B2B, Zr IV $\lambda 1598.948 \AA$ is the strongest line. A comparison with the STIS spectrum shows that a $\mathrm{Zr}$ mass fraction of $2.6 \times 10^{-6}$ (approximately 100 times solar, Grevesse et al. 2015) is the upper detection limit (Fig. 8). 
Table 4. Identified Zr lines in the UV spectrum of RE 0503-289.

\begin{tabular}{|c|c|c|c|}
\hline & & Wavelength/Å & Comment \\
\hline $\mathrm{Zr}$ & IV & 1598.948 & \\
\hline \multirow[t]{17}{*}{$\mathrm{Zr}$} & V & 1001.765 & \\
\hline & & 1002.484 & \\
\hline & & 1068.551 & blend $\mathrm{Ga} \mathrm{V}$ \\
\hline & & 1119.158 & uncertain \\
\hline & & 1200.760 & \\
\hline & & 1245.951 & \\
\hline & & 1260.909 & \\
\hline & & 1265.381 & \\
\hline & & 1303.933 & \\
\hline & & 1306.762 & \\
\hline & & 1323.826 & \\
\hline & & 1332.065 & \\
\hline & & 1355.216 & \\
\hline & & 1355.975 & \\
\hline & & 1376.544 & \\
\hline & & 1633.027 & \\
\hline & & 1725.024 & uncertain \\
\hline \multirow[t]{11}{*}{$\mathrm{Zr}$} & VI & 1053.548 & \\
\hline & & 1064.818 & \\
\hline & & 1068.663 & uncertain \\
\hline & & 1099.591 & \\
\hline & & 1118.689 & \\
\hline & & 1151.571 & \\
\hline & & 1514.568 & \\
\hline & & 1521.699 & \\
\hline & & 1591.799 & \\
\hline & & 1682.241 & \\
\hline & & 1749.350 & uncertain \\
\hline
\end{tabular}

Notes. The wavelengths correspond to those in Tables A.9-A.11.

\section{Xenon}

\subsection{Oscillator-strength calculations for $\mathrm{Xe} I \mathrm{~V}, \mathrm{~V}$, and $\mathrm{VII}$ ions}

New calculations of oscillator strengths and radiative transition probabilities in xenon ions were also performed using the HFR+CPOL method (Cowan 1981; Quinet et al. 1999, 2002).

For Xe IV, the multiconfiguration expansion included $5 \mathrm{~s}^{2} 5 \mathrm{p}^{3}$, $5 \mathrm{~s}^{2} 5 \mathrm{p}^{2} 6 \mathrm{p}, 5 \mathrm{~s}^{2} 5 \mathrm{p}^{2} n \mathrm{f}(n=4-6), 5 \mathrm{~s}^{2} 5 \mathrm{p} 5 \mathrm{~d} 6 \mathrm{~s}, 5 \mathrm{~s}^{2} 5 \mathrm{p} 5 \mathrm{~d} 6 \mathrm{~d}, 5 \mathrm{~s}^{2} 5 \mathrm{p} 6 \mathrm{~s}^{2}$, $5 \mathrm{~s}^{2} 5 \mathrm{p} 5 \mathrm{~d}^{2}, 5 \mathrm{~s}^{2} 5 \mathrm{p} 4 \mathrm{f}^{2}, 5 \mathrm{~s} 5 \mathrm{p}^{3} 6 \mathrm{~s}, 5 \mathrm{~s} 5 \mathrm{p}^{3} n \mathrm{~d}(n=5-6), 5 \mathrm{~s} 5 \mathrm{p}^{2} 4 \mathrm{f} 5 \mathrm{~d}$, and $5 \mathrm{p}^{5}$ for the odd parity, and $5 \mathrm{~s} 5 \mathrm{p}^{4}, 5 \mathrm{~s}^{2} 5 \mathrm{p}^{2} n \mathrm{~d}(n=5-6), 5 \mathrm{~s}^{2} 5 \mathrm{p}^{2} 6 \mathrm{~s}$, $5 \mathrm{~s}^{2} 5 \mathrm{p}^{2} n \mathrm{~g}(n=5-6), 5 \mathrm{~s}^{2} 5 \mathrm{p} 5 \mathrm{~d} 6 \mathrm{p}, 5 \mathrm{~s}^{2} 5 \mathrm{p} 5 \mathrm{~d} n \mathrm{f}(n=4-6), 5 \mathrm{~s} 5 \mathrm{p}^{3} 6 \mathrm{p}$, $5 \mathrm{~s} 5 \mathrm{p}^{3} n \mathrm{f}(n=4-6)$, and $5 \mathrm{~s} 5 \mathrm{p}^{2} 5 \mathrm{~d}^{2}$ for the even parity. The corepolarization effects were estimated with $\alpha_{\mathrm{d}}=0.88 \mathrm{a}$.u. and $r_{\mathrm{c}}$ $=0.86$ a.u. which correspond to a Pd-like Xe IX ionic core. The former value was taken from Fraga et al. (1976) while the latter one corresponds to the HFR mean value $\langle r\rangle$ of the outermost core orbital $(4 \mathrm{~d})$. The experimental energy levels published by Saloman (2004) were then used to optimize the radial parameters belonging to the $5 p^{3}, 5 p^{2} 6 p, 5 p^{2} 4 f, 5 s 5 p^{4}, 5 p^{2} 5 d$, and $5 p^{2} 6 s$ configurations allowing us to reach average deviations between calculated and observed energies of $137 \mathrm{~cm}^{-1}$ and $251 \mathrm{~cm}^{-1}$, for odd and even parities, respectively.

In the case of $\mathrm{Xe} \mathrm{V}$, the following sets of configurations were considered in the HFR model: $5 \mathrm{~s}^{2} 5 \mathrm{p}^{2}, 5 \mathrm{~s}^{2} 5 \mathrm{p} 6 \mathrm{p}, 5 \mathrm{~s}^{2} 5 \mathrm{p} n \mathrm{f}(n=4-$ 6), $5 \mathrm{~s}^{2} 5 \mathrm{~d} 6 \mathrm{~s}, 5 \mathrm{~s}^{2} 5 \mathrm{~d} 6 \mathrm{~d}, 5 \mathrm{~s}^{2} 6 \mathrm{~s}^{2}, 5 \mathrm{~s}^{2} 5 \mathrm{~d}^{2}, 5 \mathrm{~s}^{2} 4 \mathrm{f}^{2}, 5 \mathrm{~s}^{2} 5 \mathrm{f}^{2}, 5 \mathrm{~s}^{2} \mathrm{p}^{2} 6 \mathrm{~s}$, $5 \mathrm{~s} 5 \mathrm{p}^{2} n \mathrm{~d}(n=5-6), 5 \mathrm{~s} 5 \mathrm{p} 6 \mathrm{~s} 6 \mathrm{p}, 5 \mathrm{~s} 5 \mathrm{p} 6 \mathrm{p} n \mathrm{~d}(n=5-6), 5 \mathrm{~s} 5 \mathrm{p} 4 \mathrm{f} n \mathrm{~d}$ $(n=5-6), 5 \mathrm{p}^{4}, 5 \mathrm{p}^{3} 6 \mathrm{p}$, and $5 \mathrm{p}^{3} n \mathrm{f}(n=4-6)$ for the even parity, and $5 \mathrm{~s} 5 \mathrm{p}^{3}, 5 \mathrm{~s}^{2} 5 \mathrm{p} n \mathrm{~d}(n=5-6), 5 \mathrm{~s}^{2} 5 \mathrm{p} n \mathrm{~s}(n=6-7), 5 \mathrm{~s}^{2} 5 \mathrm{p} n \mathrm{~g}$

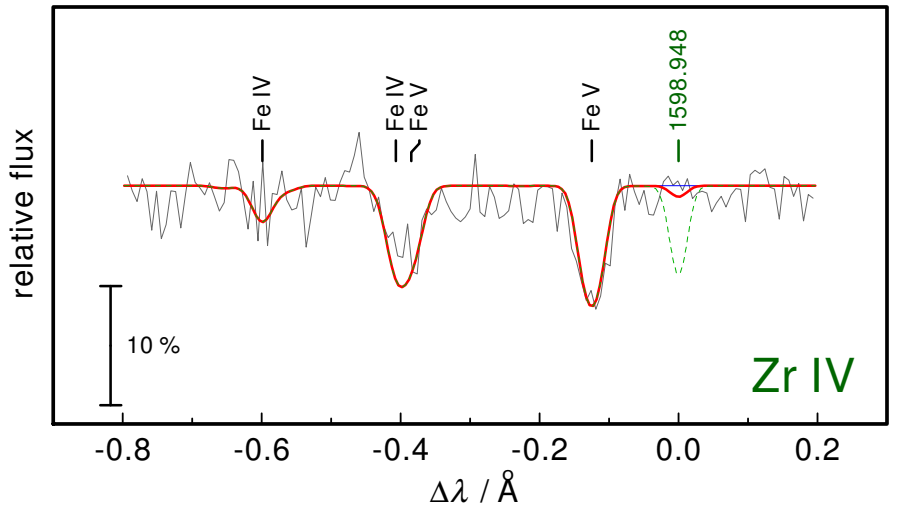

Fig. 8. Section of the STIS spectrum of G191-B2B around Zr IV $\lambda 1598.948 \AA$ compared with three synthetic spectra (thin, blue: no $\mathrm{Zr}$, thick, red: $\mathrm{Zr}$ mass fraction $=2.6 \times 10^{-6}$, dashed green: $\mathrm{Zr}=$ $\left.2.6 \times 10^{-5}\right)$.

$(n=5-6), 5 \mathrm{~s}^{2} 5 \mathrm{~d} 6 \mathrm{p}, 5 \mathrm{~s}^{2} 5 \mathrm{~d} n \mathrm{f}(n=4-6), 5 \mathrm{~s} 5 \mathrm{p}^{2} 6 \mathrm{p}, 5 \mathrm{~s} 5 \mathrm{p}^{2} n \mathrm{f}$ $(n=4-6), 5 \mathrm{~s} 5 \mathrm{p} 6 \mathrm{~s} n \mathrm{~d}(n=5-6), 5 \mathrm{~s} 5 \mathrm{p} 5 \mathrm{~d} 6 \mathrm{~d}, 5 \mathrm{~s} 5 \mathrm{p} 6 \mathrm{~s}^{2}, 5 \mathrm{~s} 5 \mathrm{p} 5 \mathrm{~d}^{2}$, $5 \mathrm{p}^{3} 6 \mathrm{~s}$, and $5 \mathrm{p}^{3} n \mathrm{~d}(n=5-6)$ for the odd parity. The same corepolarization parameters as those used for Xe IV were used and the experimental energy levels reported by Saloman (2004) and Raineri et al. (2009) were incorporated into the semi-empirical fit to adjust the radial integrals corresponding to the $5 p^{2}, 5 p 6 p$, $5 \mathrm{p} 4 \mathrm{f}, 5 \mathrm{~s} 5 \mathrm{p}^{3}, 5 \mathrm{p} 5 \mathrm{~d}, 5 \mathrm{p} 6 \mathrm{~d}, 5 \mathrm{p} 6 \mathrm{~s}$, and $5 \mathrm{p} 7 \mathrm{~s}$ configurations. In this process, we found mean deviations equal to $144 \mathrm{~cm}^{-1}$ in the even parity and $110 \mathrm{~cm}^{-1}$ in the odd parity.

For Xe VI, we used the same atomic data as those considered in one of our previous papers (Rauch et al. 2015a). More precisely, the radiative rates were taken from the work of Gallardo et al. (2015) who performed HFR+CPOL calculations including 35 odd-parity and 34 even-parity configurations, that is, $5 \mathrm{~s}^{2} n \mathrm{p}(n=5-8), 5 \mathrm{~s}^{2} n \mathrm{f}(n=4-8), 5 \mathrm{~s}^{2} n \mathrm{~h}(n=6-8)$, $5 \mathrm{~s}^{2} 8 \mathrm{k}, 5 \mathrm{p}^{2} n \mathrm{p}(n=6-8), 5 \mathrm{p}^{2} n \mathrm{f}(n=4-8), 5 \mathrm{p}^{2} n \mathrm{~h}(n=6-8)$, $5 \mathrm{p}^{2} 8 \mathrm{k}, 5 \mathrm{~s} 5 \mathrm{p} 6 \mathrm{~s}, 5 \mathrm{~s} 5 \mathrm{p} n \mathrm{~d}(n=5-6), 5 \mathrm{~s} 5 \mathrm{p} n \mathrm{~g}(n=5-6), 5 \mathrm{p}^{3}, 5 \mathrm{~s} 5 \mathrm{~d} n \mathrm{f}$ $(n=4-5), 5 \mathrm{~s} 6 \mathrm{~s} n \mathrm{f}(n=4-5)$, and $5 \mathrm{~s}^{2} \mathrm{p}^{2}, 5 \mathrm{~s}^{2} n \mathrm{~s}(n=6-8), 5 \mathrm{~s}^{2} n \mathrm{~d}$ $(n=5-8), 5 \mathrm{~s}^{2} n \mathrm{~g}(n=5-8), 5 \mathrm{~s}^{2} n \mathrm{i}(n=7-8), 5 \mathrm{p}^{2} n \mathrm{~d}(n=5-$ $8), 5 \mathrm{p}^{2} n \mathrm{~s}(n=6-8), 5 \mathrm{p}^{2} n \mathrm{~g}(n=5-8), 5 \mathrm{p}^{2} n \mathrm{i}(n=7-8), 5 \mathrm{~s} 5 \mathrm{p} n \mathrm{f}$ $(n=4-6), 5 s 4 \mathrm{f}^{2}, 5 \mathrm{~s} 5 \mathrm{f}^{2}, 5 \mathrm{~s} 5 \mathrm{p} 6 \mathrm{p}, 4 \mathrm{~d}^{9} 5 \mathrm{p}^{4}$, respectively. In this latter study, the core-polarization effects were considered with two different ionic cores, that is, a Cd-like Xe VII core with $\alpha_{\mathrm{d}}=5.80$ a.u. for the $5 \mathrm{~s}^{2} n l-5 \mathrm{~s}^{2} n^{\prime} l^{\prime}$ transitions, and a Pd-like Xe IX core with $\alpha_{\mathrm{d}}=0.99$ a.u. for all the other transitions. In their semi-empirical least-squares fitting process, Gallardo et al. (2015) achieved standard deviations with experimental energy levels of $149 \mathrm{~cm}^{-1}$ in the odd parity and $154 \mathrm{~cm}^{-1}$ in the even parity.

Finally, for Xe VII, we used the same model as the one considered by Biémont et al. (2007) extending the set of oscillator strengths to weaker transitions (up to $\log g f>-8$ ). As a reminder, these authors explicitly retained the following configurations in their configuration interaction expansions: $5 \mathrm{~s}^{2}, 5 \mathrm{p}^{2}$, $5 \mathrm{~d}^{2}, 4 \mathrm{f}^{2}, 4 \mathrm{f} n \mathrm{p}(n=5-6), 4 \mathrm{f} 6 \mathrm{f}, 4 \mathrm{f} 6 \mathrm{~h}, 5 \mathrm{~s} 6 \mathrm{~s}, 5 \mathrm{~s} n \mathrm{~d}(n=5-6), 5 \mathrm{~s} n \mathrm{~g}$ $(n=5-6), 5 \mathrm{p} n \mathrm{f}(n=5-6), 5 \mathrm{p} 6 \mathrm{p}, 5 \mathrm{p} 6 \mathrm{~h}, 5 \mathrm{~d} 6 \mathrm{~s}, 5 \mathrm{~d} 6 \mathrm{~d}$, and $5 \mathrm{~d} n \mathrm{~g}$ $(n=5-6)$ for the even parity, and $5 \mathrm{~s} n \mathrm{p}(n=5-6), 5 \mathrm{~s} n \mathrm{f}(n=4-6)$, 5s6h, 4f6s, 4f $n \mathrm{~d}(n=5-6), 4 \mathrm{f} n \mathrm{~g}(n=5-6), 5 \mathrm{p} 6 \mathrm{~s}, 5 \mathrm{p} n \mathrm{~d}(n=5-$ $6), 5 \mathrm{p} n \mathrm{~g}(n=5-6), 5 \mathrm{~d} 6 \mathrm{p}$, and $5 \mathrm{~d} n \mathrm{f}(n=5-6), 5 \mathrm{~d} 6 \mathrm{~h}$ for the odd parity. The same ionic core parameters as those used for Xe $\mathrm{IV}$ and $\mathrm{Xe} \mathrm{V}$ ions were considered and all the experimental energy levels published by Saloman (2004) were included in the semi-empirical optimization of the radial parameters belonging 


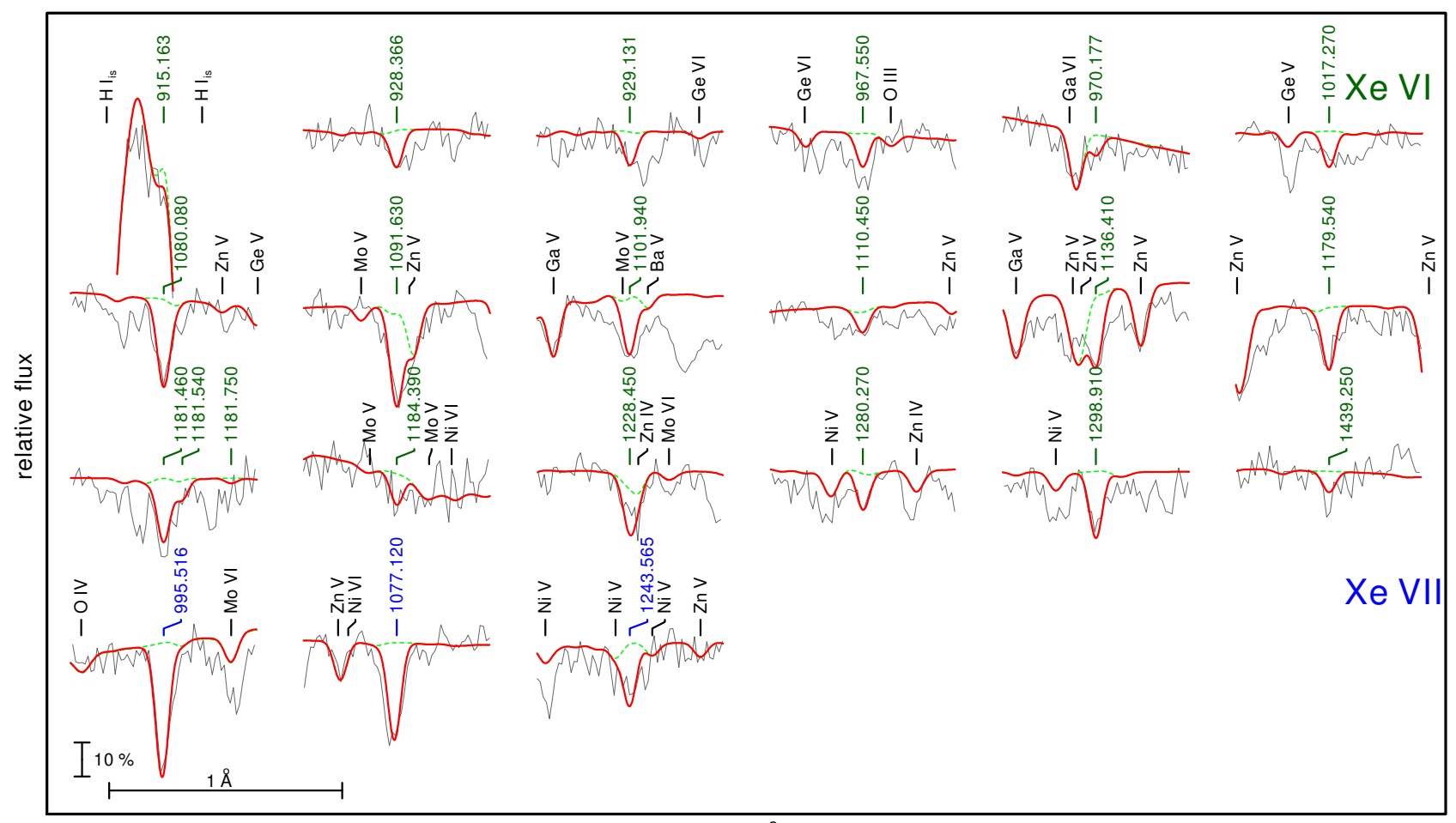

$\Delta \lambda / \AA$

Fig. 9. Identified Xe VI (top three rows) and Xe VII (bottom row) lines in the FUSE $(\lambda<1188 \AA$ ) and HST/STIS observations of RE 0503-289. The model (thick, red line) was calculated with an abundance of $\log \mathrm{Xe}=-3.9$. The dashed, green spectrum was calculated without Xe. Prominent lines are marked ("is" denotes interstellar origin), and the Xe lines are labelled with their wavelengths given by Gallardo et al. (2015) and in Table B.7.

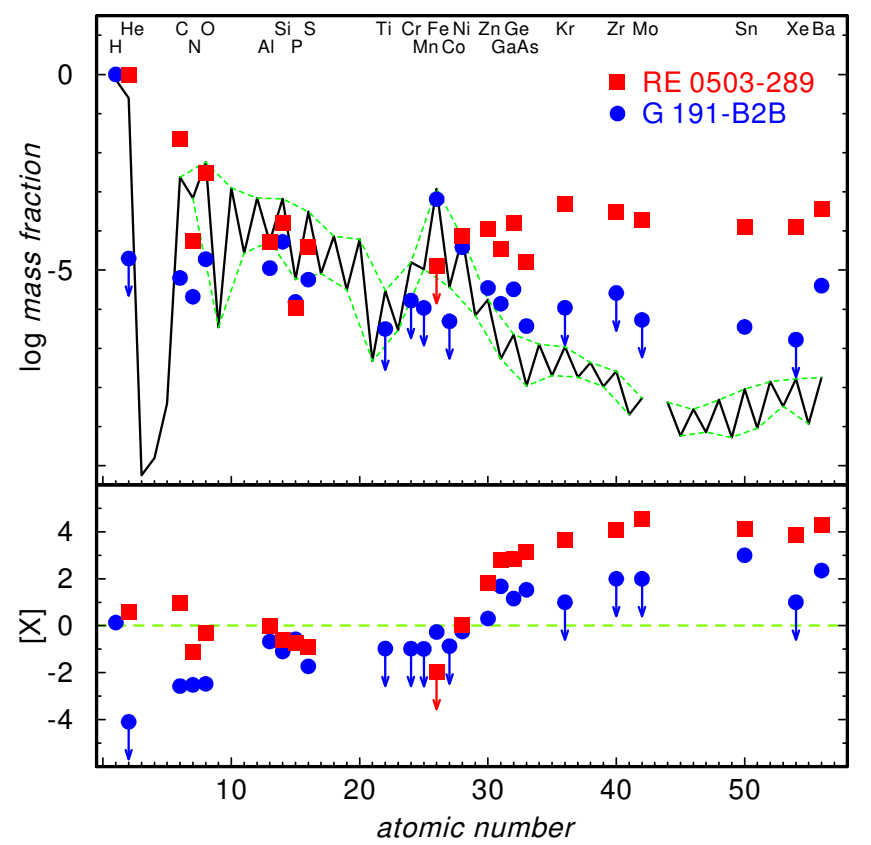

Fig. 10. Solar abundances (Asplund et al. 2009; Scott et al. 2015b,a; Grevesse et al. 2015, thick line; the dashed lines connect the elements with even and with odd atomic number) compared with the determined photospheric abundances of G191-B2B (blue circles, Rauch et al. 2013) and RE 0503-289 (red squares, Dreizler \& Werner 1996; Rauch et al. 2012, 2014a,b, 2015a,b, 2016a,b, and this work). The uncertainties of the WD abundances are, in general, approximately 0.2 dex. The arrows indicate upper limits. Top panel: abundances given as logarithmic mass fractions. Bottom panel: abundance ratios to respective solar values, $[\mathrm{X}]$ denotes $\log$ (fraction/solar fraction) of species $\mathrm{X}$. The dashed green line indicates solar abundances. to the $5 s^{2}, 5 s 6 s, 5 s 5 d, 5 s 6 d, 5 p^{2}, 4 f 5 p, 5 s 5 p, 5 s 6 p, 5 s 4 f, 5 s 5 f$, $5 \mathrm{p} 6 \mathrm{~s}$, and $5 \mathrm{p} 5 \mathrm{~d}$ configurations giving rise to standard deviations of $377 \mathrm{~cm}^{-1}$ and $250 \mathrm{~cm}^{-1}$ for even- and odd-parity levels, respectively.

The radial parameters used in our computations are summarized in Tables B.1, B.2 for the Xe IV-V ions, respectively. The calculated energy levels are compared with available experimental values in Tables B.3, B.4 while the HFR weighted oscillator strengths $(\log g f)$ and transition probabilities $\left(g A\right.$ in $\left.\mathrm{s}^{-1}\right)$ are reported in Tables B.5-B.7 for the Xe IV-V and VII ions, respectively. In the latter tables, we also give the numerical values (in $\mathrm{cm}^{-1}$ ) of lower and upper energy levels of each transition together with the corresponding wavelength (in $\AA$ ) and the $C F$, as introduced in Sect. 6.1. These tables are provided via TOSS.

\subsection{Xe line identification and abundance analysis}

In the FUSE and HST/STIS observations of RE 0503-289, we identified Xe VI-VII lines (Table 5). The observation is well reproduced by our model, calculated with a mass fraction of $\log \mathrm{Xe}=-3.9 \pm 0.2$ (Fig. 9). This is a factor of two higher than that previously determined by Werner et al. $(2012 \mathrm{~b}, \log \mathrm{Xe}=$ $-4.2 \pm 0.6)$ but agrees within their given error limits. The $\mathrm{Xe} \mathrm{VI/VII} \mathrm{ionization} \mathrm{equilibrium} \mathrm{is} \mathrm{matched} \mathrm{by} \mathrm{our} \mathrm{model.}$

\section{Results and conclusions}

To search for Al lines in the observed UV spectrum of RE 0503-289, we created an extended Al model atom for our NLTE model-atmosphere calculations. We could only identify $\mathrm{Al}$ III $\lambda \lambda 1384.130 \AA$ (Sect. 5), that was well suited to measure the $\mathrm{Al}$ abundance. It is reproduced at a solar value $(-4.28 \pm 0.2$, mass 


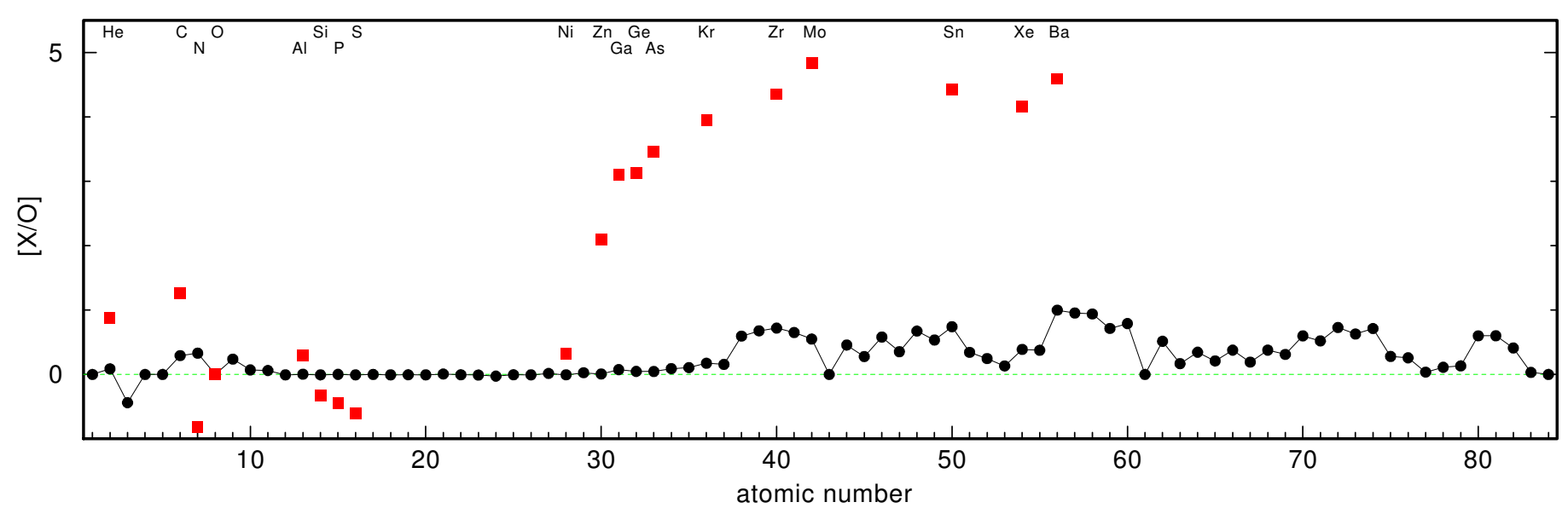

Fig. 11. Determined photospheric abundances of RE0503-289 (cf. Fig. 10) compared with predictions for surface abundances of Karakas \& Lugaro (2016, for an asymptotic giant branch (AGB) star with $M_{\text {initial }}=1.5 M_{\odot}, M_{\text {final }}=0.585 M_{\odot}$, metallicity $Z=0.014$ ). [X/O] denotes the normalized $\log [($ fraction of $\mathrm{X} /$ solar fraction of $\mathrm{X}) /($ fraction of $\mathrm{O} /$ solar fraction of $\mathrm{O})]$ mass ratio. The dashed green line indicates the solar ratio.

Table 5. Identified Xe lines in the UV spectrum of RE 0503-289.

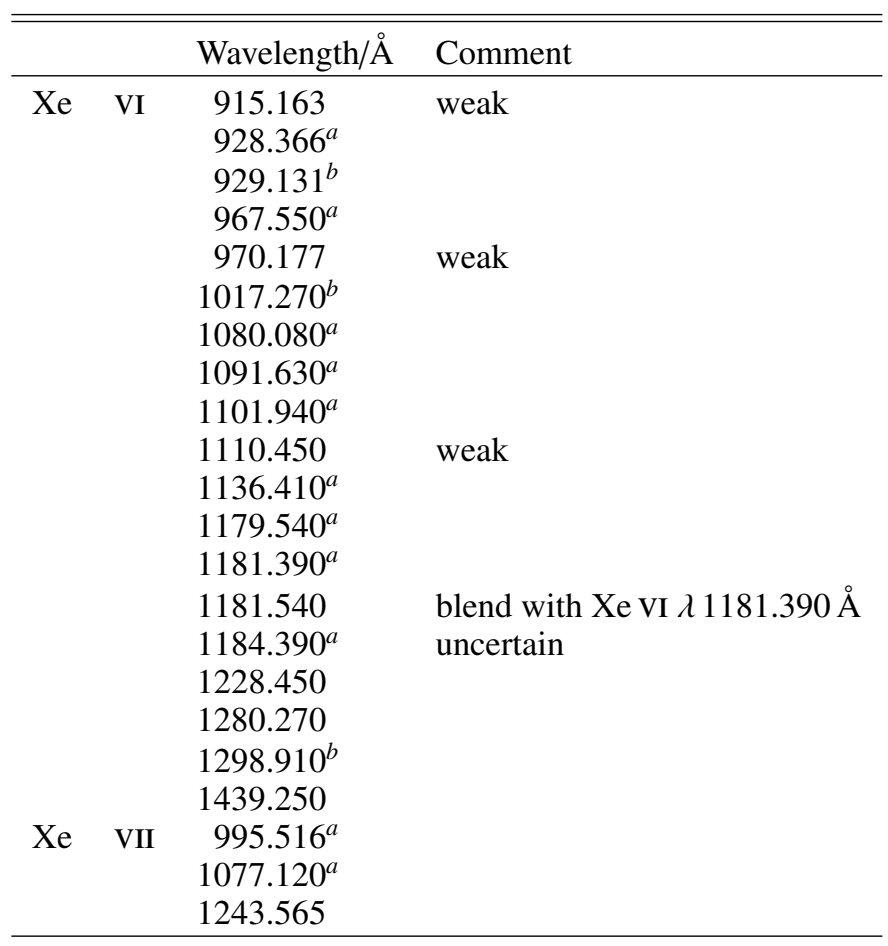

Notes. The wavelengths correspond to those given in Gallardo et al. (2015) and in Table B.7 for Xe VI and Xe VII, respectively. ${ }^{(a)}$ Identified by Werner et al. (2012b); ${ }^{(b)}$ identified by Rauch et al. (2015a).

fraction). This needs to be verified once better observations are available.

We identified $\mathrm{Zr}$ IV-VI lines in the observed high-resolution UV spectra RE 0503-289 (Table 4). These were well modeled using our newly calculated Zr IV-VII oscillator strengths. We determined a photospheric abundance of $\log \mathrm{Zr}=-3.52 \pm 0.2$ (mass fraction, $1.5-4.8 \times 10^{-4}, 5775-14480$ times the solar abundance). This highly supersolar $\mathrm{Zr}$ abundance corresponds to the high abundances of other trans-iron elements in RE 0503-289 (Fig. 10). The Zr IV/v/VI ionization equilibria are well matched by our model $\left(T_{\text {eff }}=70000 \mathrm{~K}, \log g=7.5\right)$.
In addition to the previously discovered Xe VI-VII lines in the UV spectrum of RE0503-289, we identified five new Xe VI lines. All identified Xe lines are well matched by our model with an abundance of $\log \mathrm{Xe}=-3.88 \pm 0.2$ (mass fraction, $0.8-2.1 \times 10^{-4}, 4985-12520$ times the solar abundance). This highly supersolar Xe abundance is in line with abundances of other trans-iron elements in RE 0503-289 (Fig. 10).

The amount of trans-iron elements in the photosphere of RE 0503-289 strongly exceeds the yields of nucleosynthesis on the asymptotic giant branch (Fig 11). It is likely that radiative levitation is working efficiently in RE 0503-289 (Rauch et al. $2016 a$ ), increasing abundances by up to 4 dex compared with solar values.

The identification of lines of $\mathrm{Zr}$ and $\mathrm{Xe}$ and their precise abundance determinations only became possible after reliable transition probabilities for Zr IV-VII, Xe IV-V, and Xe VII were computed. Calculations for other, highly-ionized trans-iron elements are necessary to search for their lines and to measure their abundances.

The search for $\mathrm{Zr}$ and $\mathrm{Xe}$ lines in the UV spectrum of G191-B2B was entirely negative. We established an upper $\mathrm{Zr}$ abundance limit of approximately 100 times solar and confirmed the previously found upper limit for Xe of approximately 10 times solar (Rauch et al. 2016a).

Acknowledgements. T.R. and D.H. are supported by the German Aerospace Center (DLR, grants 05 OR 1507 and 50 OR 1501, respectively). The GAVO project had been supported by the Federal Ministry of Education and Research (BMBF) at Tübingen (05 AC 6 VTB, 05 AC 11 VTB) and is funded at Heidelberg (05 AC 11 VH3). Financial support from the Belgian FRS-FNRS is also acknowledged. P.Q. is research director of this organization. Some of the data presented in this paper were obtained from the Mikulski Archive for Space Telescopes (MAST). STScI is operated by the Association of Universities for Research in Astronomy, Inc., under NASA contract NAS5-26555. Support for MAST for non-HST data is provided by the NASA Office of Space Science via grant NNX09AF08G and by other grants and contracts. This research has made use of NASA's Astrophysics Data System and the SIMBAD database, operated at CDS, Strasbourg, France. The TOSS service (http://dc.g-vo.org/TOSS) that provides weighted oscillator strengths and transition probabilities was constructed as part of the activities of the German Astrophysical Virtual Observatory.

\section{References}

Asplund, M., Grevesse, N., Sauval, A. J., \& Scott, P. 2009, ARA\&A, 47, 481 Biémont, É., Clar, M., Fivet, V., et al. 2007, Eur. Phys. J. D, 44, 23 
T. Rauch et al.: Stellar laboratories. VIII.

Bohlin, R. C. 2007, in The Future of Photometric, Spectrophotometric and Polarimetric Standardization, ed. C. Sterken, ASP Conf. Ser., 364, 315

Cowan, R. D. 1981, in The theory of atomic structure and spectra (Berkeley, CA: University of California Press)

Cowley, C. R. 1970, in The theory of stellar spectra (New York: Gordon \& Breach)

Cowley, C. R. 1971, The Observatory, 91, 139

Dreizler, S., \& Werner, K. 1996, A\&A, 314, 217

Fraga, S., Karwowski, J., \& Saxena, K. M. S. 1976, in Handbook of Atomic Data (Amsterdam: Elsevier)

Gallardo, M., Raineri, M., Reyna Almandos, J., Pagan, C. J. B., \& Abrahão, R. A. 2015, ApJS, 216, 11

Grevesse, N., Scott, P., Asplund, M., \& Sauval, A. J. 2015, A\&A, 573, A27

Holberg, J. B., Barstow, M. A., \& Sion, E. M. 1998, ApJS, 119, 207

Hubeny, I., Hummer, D. G., \& Lanz, T. 1994, A\&A, 282, 151

Hummer, D. G., \& Mihalas, D. 1988, ApJ, 331, 794

Karakas, A. I., \& Lugaro, M. 2016, ApJ, 825, 26

Khan, Z. A., Rahimullah, K., \& Chaghtai, M. S. Z. 1981, Phys. Scr., 23, 843

Khan, Z. A., Chaghtai, M. S. Z., \& Rahimullah, K. 1983, J. Phys. B: At. Mol. Phys., 16, 1685

Lemoine, M., Vidal-Madjar, A., Hébrard, G., et al. 2002, ApJS, 140, 67

McCook, G. P., \& Sion, E. M. 1999a, ApJS, 121, 1

McCook, G. P., \& Sion, E. M. 1999b, VizieR Online Data Catalog: III/210

Müller-Ringat, E. 2013, Dissertation, University of Tübingen, Germany, http: //www.ivoa.net/documents/SimDM/index.html

Quinet, P., Palmeri, P., Biémont, É., et al. 1999, MNRAS, 307, 934

Quinet, P., Palmeri, P., Biémont, É., et al. 2002, J. Alloys Comp., 344, 255

Rahimullah, K., Chaghtai, M. S. Z., \& Khatoon, S. 1978, Physica Scripta, 18, 96
Raineri, M., Gallardo, M., Padilla, S., \& Reyna Almandos, J. 2009, J. Phys. B, 42, 205004

Rauch, T., \& Deetjen, J. L. 2003, in Stellar Atmosphere Modeling, eds. I. Hubeny, D. Mihalas, \& K. Werner, ASP Conf. Ser., 288, 103

Rauch, T., Werner, K., Biémont, É., Quinet, P., \& Kruk, J. W. 2012, A\&A, 546, A55

Rauch, T., Werner, K., Bohlin, R., \& Kruk, J. W. 2013, A\&A, 560, A106

Rauch, T., Werner, K., Quinet, P., \& Kruk, J. W. 2014a, A\&A, 564, A41

Rauch, T., Werner, K., Quinet, P., \& Kruk, J. W. 2014b, A\&A, 566, A10

Rauch, T., Hoyer, D., Quinet, P., Gallardo, M., \& Raineri, M. 2015a, A\&A, 577, A88

Rauch, T., Werner, K., Quinet, P., \& Kruk, J. W. 2015b, A\&A, 577, A6

Rauch, T., Quinet, P., Hoyer, D., et al. 2016a, A\&A, 587, A39

Rauch, T., Quinet, P., Hoyer, D., et al. 2016b, A\&A, 590, A128

Reader, J., \& Acquista, N. 1976, J. Opt. Soc. Am., 66, 896

Reader, J., \& Acquista, N. 1979, J. Opt. Soc. Am., 69, 239

Reader, J., \& Acquista, N. 1997, J. Opt. Soc. Am. B, 14, 1328

Reader, J., \& Lindsay, M. D. 2016, Phys. Scr., 91, 02540

Saloman, E. B. 2004, J. Phys. Chem. Ref. Data, 33, 765

Scott, P., Asplund, M., Grevesse, N., Bergemann, M., \& Sauval, A. J. 2015a, A\&A, 573, A26

Scott, P., Grevesse, N., Asplund, M., et al. 2015b, A\&A, 573, A25

Werner, K., Deetjen, J. L., Dreizler, S., et al. 2003, in Stellar Atmosphere Modeling, eds. I. Hubeny, D. Mihalas, \& K. Werner, ASP Conf. Ser., 288, 31

Werner, K., Dreizler, S., \& Rauch, T. 2012a, Astrophysics Source Code Library [record ascl: 1212.015]

Werner, K., Rauch, T., Ringat, E., \& Kruk, J. W. 2012b, ApJ, 753, L7 


\section{Appendix A: Additional tables for zirconium}

Table A.1. Radial parameters (in $\mathrm{cm}^{-1}$ ) adopted for the calculations in $\mathrm{Zr}$ IV.

\begin{tabular}{|c|c|c|c|c|c|}
\hline Configuration & Parameter & HFR & Fitted & Ratio & Note $^{a}$ \\
\hline \multicolumn{6}{|c|}{ Even parity } \\
\hline \multirow[t]{2}{*}{$4 d$} & $E_{\mathrm{av}}$ & 3588 & 3569 & & \\
\hline & $\zeta_{4 d}$ & 515 & 503 & 0.975 & \\
\hline \multirow[t]{2}{*}{$5 \mathrm{~d}$} & $E_{\mathrm{av}}$ & 149752 & 147800 & & \\
\hline & $\zeta_{5 d}$ & 116 & 136 & 1.172 & \\
\hline \multirow[t]{2}{*}{$6 \mathrm{~d}$} & $E_{\mathrm{av}}$ & 200505 & 198248 & & \\
\hline & $\zeta_{6 d}$ & 53 & 64 & 1.209 & \\
\hline $5 \mathrm{~s}$ & $E_{\mathrm{av}}$ & 42289 & 41703 & & \\
\hline $6 s$ & $E_{\mathrm{av}}$ & 155574 & 152690 & & \\
\hline $7 \mathrm{~s}$ & $E_{\mathrm{av}}$ & 202666 & 200188 & & \\
\hline $8 \mathrm{~s}$ & $E_{\mathrm{av}}$ & 227115 & 224846 & & \\
\hline \multirow[t]{2}{*}{$5 \mathrm{~g}$} & $E_{\mathrm{av}}$ & 208796 & 207068 & & \\
\hline & $\zeta_{5 g}$ & 0.4 & 0.4 & 1.000 & $\mathrm{~F}$ \\
\hline \multirow[t]{2}{*}{$6 g$} & $E_{\mathrm{av}}$ & 230427 & 228611 & & \\
\hline & $\zeta_{6 g}$ & 0.2 & 0.2 & 1.000 & $\mathrm{~F}$ \\
\hline \multirow[t]{2}{*}{$7 \mathrm{~g}$} & $E_{\mathrm{av}}$ & 243494 & 241615 & & \\
\hline & $\zeta_{7 g}$ & 0.1 & 0.1 & 1.000 & $\mathrm{~F}$ \\
\hline \multirow[t]{2}{*}{$8 \mathrm{~g}$} & $E_{\mathrm{av}}$ & 251971 & 250056 & & \\
\hline & $\zeta_{8 g}$ & 0.1 & 0.1 & 1.000 & $\mathrm{~F}$ \\
\hline \multirow[t]{2}{*}{$9 \mathrm{~g}$} & $E_{\mathrm{av}}$ & 257782 & 255844 & & \\
\hline & $\zeta_{9 g}$ & 0.0 & 0.0 & 1.000 & $\mathrm{~F}$ \\
\hline \multicolumn{6}{|c|}{ Odd parity } \\
\hline \multirow[t]{2}{*}{$5 p$} & $E_{\mathrm{av}}$ & 86720 & 85912 & & \\
\hline & $\zeta_{5 p}$ & 1388 & 1661 & 1.197 & \\
\hline \multirow[t]{2}{*}{$6 p$} & $E_{\mathrm{av}}$ & 173349 & 170865 & & \\
\hline & $\zeta_{6 p}$ & 567 & 668 & 1.178 & \\
\hline \multirow[t]{2}{*}{$7 \mathrm{p}$} & $E_{\mathrm{av}}$ & 211618 & 209318 & & \\
\hline & $\zeta_{7 p}$ & 290 & 341 & 1.174 & \\
\hline \multirow[t]{2}{*}{$4 \mathrm{f}$} & $E_{\mathrm{av}}$ & 162823 & 161581 & & \\
\hline & $\zeta_{4 f}$ & 2.3 & 2.3 & 1.000 & $\mathrm{~F}$ \\
\hline \multirow[t]{2}{*}{$5 f$} & $E_{\mathrm{av}}$ & 205133 & 202889 & & \\
\hline & $\zeta_{5 f}$ & 1.3 & 1.3 & 1.000 & $\mathrm{~F}$ \\
\hline \multirow[t]{2}{*}{$6 f$} & $E_{\mathrm{av}}$ & 228142 & 225772 & & \\
\hline & $\zeta_{6 f}$ & 0.8 & 0.8 & 1.000 & $\mathrm{~F}$ \\
\hline \multirow[t]{2}{*}{$6 \mathrm{~h}$} & $E_{\mathrm{av}}$ & 230751 & 228744 & & \\
\hline & $\zeta_{6 h}$ & 0.1 & 0.1 & 1.000 & $\mathrm{~F}$ \\
\hline
\end{tabular}

Notes. ${ }^{(a)}$ F: Fixed parameter value. 
T. Rauch et al.: Stellar laboratories. VIII.

Table A.2. Radial parameters (in $\mathrm{cm}^{-1}$ ) adopted for the calculations in $\mathrm{Zr} \mathrm{V}$.

\begin{tabular}{|c|c|c|c|c|c|}
\hline Configuration & Parameter & HFR & Fitted & Ratio & Note $^{a}$ \\
\hline \multicolumn{6}{|c|}{ Even parity } \\
\hline $4 p^{6}$ & $E_{\mathrm{av}}$ & 17448 & 17850 & & \\
\hline \multirow[t]{6}{*}{$4 p^{5} 5 p$} & $E_{\mathrm{av}}$ & 387307 & 386625 & & \\
\hline & $\zeta_{4 p}$ & 9939 & 10310 & 1.037 & \\
\hline & $\zeta_{5 p}$ & 1865 & 2181 & 1.169 & \\
\hline & $\mathrm{F}^{2}(4 \mathrm{p}, 5 \mathrm{p})$ & 22698 & 18416 & 0.811 & \\
\hline & $G^{0}(4 p, 5 p)$ & 4681 & 3978 & 0.850 & $\mathrm{R} 1$ \\
\hline & $G^{2}(4 p, 5 p)$ & 6345 & 5392 & 0.850 & $\mathrm{R} 1$ \\
\hline \multirow[t]{6}{*}{$4 p^{5} 6 p$} & $E_{\mathrm{av}}$ & 502342 & 500684 & & \\
\hline & $\zeta_{4 p}$ & 9978 & 10094 & 1.012 & \\
\hline & $\zeta_{6 p}$ & 806 & 806 & 1.000 & $\mathrm{~F}$ \\
\hline & $\mathrm{F}^{2}(4 \mathrm{p}, 6 \mathrm{p})$ & 8810 & 9530 & 1.082 & \\
\hline & $G^{0}(4 p, 6 p)$ & 1627 & 1480 & 0.909 & $\mathrm{R} 2$ \\
\hline & $G^{2}(4 p, 6 p)$ & 2338 & 2127 & 0.909 & $\mathrm{R} 2$ \\
\hline \multirow[t]{6}{*}{$4 p^{5} 4 f$} & $E_{\mathrm{av}}$ & 467645 & 466814 & & \\
\hline & $\zeta_{4 p}$ & 9921 & 10271 & 1.035 & \\
\hline & $\zeta_{4 f}$ & 5.6 & 5.6 & 1.000 & $\mathrm{~F}$ \\
\hline & $\mathrm{F}^{2}(4 \mathrm{p}, 4 \mathrm{f})$ & 26008 & 22351 & 0.859 & \\
\hline & $G^{2}(4 p, 4 f)$ & 15949 & 15868 & 0.995 & R3 \\
\hline & $G^{4}(4 p, 4 f)$ & 10543 & 10489 & 0.995 & R3 \\
\hline \multirow[t]{3}{*}{$4 s 4 p^{6} 4 d$} & $E_{\mathrm{av}}$ & 489915 & 486506 & & \\
\hline & $\zeta_{4 d}$ & 632 & 602 & 0.951 & \\
\hline & $\mathrm{G}^{2}(4 \mathrm{~s}, 4 \mathrm{~d})$ & 59108 & 55453 & 0.938 & \\
\hline \multirow[t]{2}{*}{$4 p^{5} 4 f-4 s 4 p^{6} 4 d$} & $\mathrm{R}^{1}(4 \mathrm{~s} 4 \mathrm{f} ; 4 \mathrm{p} 4 \mathrm{~d})$ & 48624 & 41323 & 0.850 & $\mathrm{R} 4$ \\
\hline & $\mathrm{R}^{2}(4 \mathrm{~s} 4 \mathrm{f} ; 4 \mathrm{p} 4 \mathrm{~d})$ & 29168 & 24793 & 0.850 & $\mathrm{R} 4$ \\
\hline \multicolumn{6}{|c|}{ Odd parity } \\
\hline \multirow[t]{6}{*}{$4 p^{5} 4 d$} & $E_{\mathrm{av}}$ & 282268 & 268099 & & \\
\hline & $\zeta_{4 p}$ & 9573 & 9593 & 1.002 & \\
\hline & $\zeta_{4 d}$ & 616 & 651 & 1.057 & \\
\hline & $\mathrm{F}^{2}(4 \mathrm{p}, 4 \mathrm{~d})$ & 65494 & 57294 & 0.875 & \\
\hline & $\mathrm{G}^{1}(4 \mathrm{p}, 4 \mathrm{~d})$ & 81132 & 66326 & 0.818 & \\
\hline & $\mathrm{G}^{3}(4 \mathrm{p}, 4 \mathrm{~d})$ & 50008 & 44565 & 0.891 & \\
\hline \multirow[t]{6}{*}{$4 p^{5} 5 d$} & $E_{\mathrm{av}}$ & 479226 & 463036 & & \\
\hline & $\zeta_{4 p}$ & 9933 & 10320 & 1.039 & \\
\hline & $\zeta_{5 d}$ & 163 & 194 & 1.185 & \\
\hline & $\mathrm{F}^{2}(4 \mathrm{p}, 5 \mathrm{~d})$ & 16341 & 13181 & 0.807 & \\
\hline & $\mathrm{G}^{1}(4 \mathrm{p}, 5 \mathrm{~d})$ & 9999 & 6618 & 0.662 & \\
\hline & $G^{3}(4 p, 5 d)$ & 7140 & 6306 & 0.883 & \\
\hline \multirow[t]{6}{*}{$4 p^{5} 6 d$} & $E_{\mathrm{av}}$ & 551860 & 535375 & & \\
\hline & $\zeta_{4 p}$ & 9974 & 10464 & 1.049 & \\
\hline & $\zeta_{6 d}$ & 77 & 77 & 1.000 & $\mathrm{~F}$ \\
\hline & $\mathrm{F}^{2}(4 \mathrm{p}, 6 \mathrm{~d})$ & 7018 & 3966 & 0.565 & R5 \\
\hline & $G^{1}(4 p, 6 d)$ & 3816 & 2156 & 0.565 & $\mathrm{R} 5$ \\
\hline & $G^{3}(4 p, 6 d)$ & 2854 & 1613 & 0.565 & $\mathrm{R} 5$ \\
\hline \multirow[t]{6}{*}{$4 p^{5} 7 d$} & $E_{\mathrm{av}}$ & 589057 & 573664 & & \\
\hline & $\zeta_{4 p}$ & 9989 & 9989 & 1.000 & $\mathrm{~F}$ \\
\hline & $\zeta_{7 d}$ & 43 & 43 & 1.000 & $\mathrm{~F}$ \\
\hline & $\mathrm{F}^{2}(4 \mathrm{p}, 7 \mathrm{~d})$ & 3731 & 2109 & 0.565 & R5 \\
\hline & $\mathrm{G}^{1}(4 \mathrm{p}, 7 \mathrm{~d})$ & 1940 & 1096 & 0.565 & R5 \\
\hline & $G^{3}(4 p, 7 d)$ & 1484 & 839 & 0.565 & $\mathrm{R} 5$ \\
\hline \multirow[t]{3}{*}{$4 p^{5} 5 s$} & $E_{\mathrm{av}}$ & 349759 & 335259 & & \\
\hline & $\zeta_{4 p}$ & 9867 & 10182 & 1.032 & \\
\hline & $\mathrm{G}^{1}(4 \mathrm{p}, 5 \mathrm{~s})$ & 7881 & 7278 & 0.923 & \\
\hline $4 p^{5} 6 s$ & $E_{\mathrm{av}}$ & 495108 & 478170 & & \\
\hline
\end{tabular}

Notes. ${ }^{(a)}$ F: Fixed parameter value; $\mathrm{R} n$ : ratios of these parameters have been fixed in the fitting process. 
Table A.2. continued.

\begin{tabular}{|c|c|c|c|c|c|}
\hline Configuration & Parameter & HFR & Fitted & Ratio & Note $^{a}$ \\
\hline \multirow{5}{*}{$4 p^{5} 7 \mathrm{~s}$} & $\zeta_{4 p}$ & 9959 & 10316 & 1.036 & \\
\hline & $\mathrm{G}^{1}(4 \mathrm{p}, 6 \mathrm{~s})$ & 2433 & 2132 & 0.876 & \\
\hline & $E_{\mathrm{av}}$ & 558823 & 542240 & & \\
\hline & $\zeta_{4 p}$ & 9984 & 10360 & 1.038 & \\
\hline & $\mathrm{G}^{1}(4 \mathrm{p}, 7 \mathrm{~s})$ & 1115 & 980 & 0.879 & \\
\hline \multirow[t]{3}{*}{$4 p^{5} 8 s$} & $E_{\mathrm{av}}$ & 592851 & 576592 & & \\
\hline & $\zeta_{4 p}$ & 9994 & 9994 & 1.000 & $\mathrm{~F}$ \\
\hline & $\mathrm{G}^{1}(4 \mathrm{p}, 8 \mathrm{~s})$ & 613 & 552 & 0.900 & $\mathrm{~F}$ \\
\hline \multirow[t]{3}{*}{$4 p^{5} 9 s$} & $E_{\mathrm{av}}$ & 613233 & 596840 & & \\
\hline & $\zeta_{4 p}$ & 9998 & 9998 & 1.000 & $\mathrm{~F}$ \\
\hline & $\mathrm{G}^{1}(4 \mathrm{p}, 9 \mathrm{~s})$ & 375 & 337 & 0.900 & $\mathrm{~F}$ \\
\hline \multirow[t]{3}{*}{$4 p^{5} 10 s$} & $E_{\mathrm{av}}$ & 626415 & 610078 & & \\
\hline & $\zeta_{4 p}$ & 10001 & 10001 & 1.000 & $\mathrm{~F}$ \\
\hline & $\mathrm{G}^{1}(4 \mathrm{p}, 10 \mathrm{~s})$ & 247 & 222 & 0.900 & $\mathrm{~F}$ \\
\hline \multirow[t]{6}{*}{$4 p^{5} 5 g$} & $E_{\mathrm{av}}$ & 558379 & 542891 & & \\
\hline & $\zeta_{4 p}$ & 10004 & 10394 & 1.039 & \\
\hline & $\zeta_{5 g}$ & 0.8 & 0.8 & 1.000 & $\mathrm{~F}$ \\
\hline & $\mathrm{F}^{2}(4 \mathrm{p}, 5 \mathrm{~g})$ & 4855 & 4142 & 0.853 & R6 \\
\hline & $G^{3}(4 p, 5 g)$ & 392 & 335 & 0.853 & R6 \\
\hline & $G^{5}(4 p, 5 g)$ & 277 & 236 & 0.853 & R6 \\
\hline \multirow[t]{6}{*}{$4 p^{5} 6 g$} & $E_{\mathrm{av}}$ & 592345 & 576588 & & \\
\hline & $\zeta_{4 p}$ & 10004 & 10388 & 1.038 & \\
\hline & $\zeta_{6 g}$ & 0.4 & 0.4 & 1.000 & $\mathrm{~F}$ \\
\hline & $\mathrm{F}^{2}(4 \mathrm{p}, 6 \mathrm{~g})$ & 2776 & 2436 & 0.877 & R7 \\
\hline & $\mathrm{G}^{3}(4 \mathrm{p}, 6 \mathrm{~g})$ & 358 & 314 & 0.877 & R7 \\
\hline & $\mathrm{G}^{5}(4 \mathrm{p}, 6 \mathrm{~g})$ & 253 & 222 & 0.877 & R7 \\
\hline \multirow[t]{3}{*}{$4 s 4 p^{6} 5 p$} & $E_{\mathrm{av}}$ & 629514 & 612875 & & \\
\hline & $\zeta_{5 p}$ & 1879 & 1879 & 1.000 & $\mathrm{~F}$ \\
\hline & $\mathrm{G}^{1}(4 \mathrm{~s}, 5 \mathrm{p})$ & 6870 & 6183 & 0.900 & $\mathrm{~F}$ \\
\hline \multirow[t]{2}{*}{$4 p^{5} 4 d-4 p^{5} 5 s$} & $\mathrm{R}^{2}(4 \mathrm{p} 4 \mathrm{~d} ; 4 \mathrm{p} 5 \mathrm{~s})$ & -8924 & -5044 & 0.565 & $\mathrm{R} 8$ \\
\hline & $\mathrm{R}^{1}(4 \mathrm{p} 4 \mathrm{~d} ; 4 \mathrm{p} 5 \mathrm{~s})$ & -1482 & -837 & 0.565 & $\mathrm{R} 8$ \\
\hline
\end{tabular}


T. Rauch et al.: Stellar laboratories. VIII.

Table A.3. Radial parameters (in $\mathrm{cm}^{-1}$ ) adopted for the calculations in $\mathrm{Zr}$ VI.

\begin{tabular}{|c|c|c|c|c|c|}
\hline Configuration & Parameter & HFR & Fitted & Ratio & Note $^{a}$ \\
\hline \multicolumn{6}{|c|}{ Odd parity } \\
\hline \multirow[t]{2}{*}{$4 p^{5}$} & $E_{\mathrm{av}}$ & 22997 & 23322 & & \\
\hline & $\zeta_{4 p}$ & 10007 & 10580 & 1.057 & \\
\hline \multirow[t]{8}{*}{$4 p^{4} 5 p$} & $E_{\mathrm{av}}$ & 461912 & 446765 & & \\
\hline & $\mathrm{F}^{2}(4 \mathrm{p}, 4 \mathrm{p})$ & 84088 & 79559 & 0.946 & \\
\hline & $\alpha$ & 0 & -651 & & \\
\hline & $\zeta_{4 p}$ & 10577 & 10907 & 1.031 & \\
\hline & $\zeta_{5 p}$ & 2382 & 2701 & 1.134 & \\
\hline & $\mathrm{F}^{2}(4 \mathrm{p}, 5 \mathrm{p})$ & 26052 & 21472 & 0.824 & \\
\hline & $G^{0}(4 p, 5 p)$ & 5535 & 4696 & 0.848 & \\
\hline & $\mathrm{G}^{2}(4 \mathrm{p}, 5 \mathrm{p})$ & 7459 & 6664 & 0.893 & \\
\hline \multicolumn{6}{|c|}{ Even parity } \\
\hline $4 s 4 p^{6}$ & $E_{\mathrm{av}}$ & 251206 & 224383 & & \\
\hline \multirow{8}{*}{$4 p^{4} 4 d$} & $E_{\mathrm{av}}$ & 289403 & 291464 & & \\
\hline & $\mathrm{F}^{2}(4 \mathrm{p}, 4 \mathrm{p})$ & 82744 & 78447 & 0.948 & \\
\hline & $\alpha$ & 0 & -450 & & \\
\hline & $\zeta_{4 p}$ & 10187 & 10521 & 1.033 & \\
\hline & $\zeta_{4 d}$ & 721 & 854 & 1.184 & \\
\hline & $\mathrm{F}^{2}(4 \mathrm{p}, 4 \mathrm{~d})$ & 69677 & 62179 & 0.892 & \\
\hline & $\mathrm{G}^{1}(4 \mathrm{p}, 4 \mathrm{~d})$ & 86802 & 72077 & 0.831 & \\
\hline & $\mathrm{G}^{3}(4 \mathrm{p}, 4 \mathrm{~d})$ & 53829 & 45721 & 0.849 & \\
\hline \multirow[t]{8}{*}{$4 p^{4} 5 d$} & $E_{\mathrm{av}}$ & 536543 & 535860 & & \\
\hline & $\mathrm{F}^{2}(4 \mathrm{p}, 4 \mathrm{p})$ & 84140 & 77928 & 0.926 & \\
\hline & $\alpha$ & 0 & -450 & & $\mathrm{~F}$ \\
\hline & $\zeta_{4 p}$ & 10569 & 10891 & 1.030 & \\
\hline & $\zeta_{5 d}$ & 217 & 259 & 1.191 & \\
\hline & $\mathrm{F}^{2}(4 \mathrm{p}, 5 \mathrm{~d})$ & 19555 & 16945 & 0.867 & \\
\hline & $\mathrm{G}^{1}(4 \mathrm{p}, 5 \mathrm{~d})$ & 10870 & 8250 & 0.759 & $\mathrm{R} 1$ \\
\hline & $G^{3}(4 p, 5 d)$ & 8037 & 6100 & 0.759 & $\mathrm{R} 1$ \\
\hline \multirow[t]{5}{*}{$4 p^{4} 5 s$} & $E_{\mathrm{av}}$ & 386802 & 387950 & & \\
\hline & $\mathrm{F}^{2}(4 \mathrm{p}, 4 \mathrm{p})$ & 83739 & 79833 & 0.953 & \\
\hline & $\alpha$ & 0 & -665 & & \\
\hline & $\zeta_{4 p}$ & 10498 & 10846 & 1.033 & \\
\hline & $\mathrm{G}^{1}(4 \mathrm{p}, 5 \mathrm{~s})$ & 8725 & 7618 & 0.873 & \\
\hline \multirow[t]{5}{*}{$4 p^{4} 6 s$} & $E_{\mathrm{av}}$ & 564837 & 564005 & & \\
\hline & $F^{2}(4 p, 4 p)$ & 84213 & 81311 & 0.965 & \\
\hline & $\alpha$ & 0 & -332 & & \\
\hline & $\zeta_{4 p}$ & 10600 & 11164 & 1.053 & \\
\hline & $\mathrm{G}^{1}(4 \mathrm{p}, 6 \mathrm{~s})$ & 2787 & 2372 & 0.851 & \\
\hline $4 s 4 p^{6}-4 p^{4} 4 d$ & $\mathrm{R}^{1}(4 \mathrm{p} 4 \mathrm{p} ; 4 \mathrm{~s} 4 \mathrm{~d})$ & 96078 & 72916 & 0.759 & $\mathrm{R} 2$ \\
\hline $4 s 4 p^{6}-4 p^{4} 5 d$ & $\mathrm{R}^{1}(4 \mathrm{p} 4 \mathrm{p} ; 4 \mathrm{~s} 5 \mathrm{~d})$ & 32299 & 24513 & 0.759 & $\mathrm{R} 2$ \\
\hline
\end{tabular}

Notes. ${ }^{(a)}$ F: Fixed parameter value; $\mathrm{R} n$ : ratios of these parameters have been fixed in the fitting process. 
Table A.4. Radial parameters (in $\mathrm{cm}^{-1}$ ) adopted for the calculations in $\mathrm{Zr}$ VII.

\begin{tabular}{|c|c|c|c|c|c|}
\hline Configuration & Parameter & HFR & Fitted & Ratio & Note $^{a}$ \\
\hline \multicolumn{6}{|c|}{ Even parity } \\
\hline \multirow[t]{4}{*}{$4 p^{4}$} & $E_{\mathrm{av}}$ & 23653 & 33968 & & \\
\hline & $\mathrm{F}^{2}(4 \mathrm{p}, 4 \mathrm{p})$ & 84430 & 65839 & 0.780 & \\
\hline & $\alpha$ & 0 & 646 & & \\
\hline & $\zeta_{4 p}$ & 10658 & 11259 & 1.056 & \\
\hline \multirow[t]{8}{*}{$4 p^{3} 5 p$} & $E_{\mathrm{av}}$ & 516191 & 514481 & & \\
\hline & $\mathrm{F}^{2}(4 \mathrm{p}, 4 \mathrm{p})$ & 86163 & 82914 & 0.962 & \\
\hline & $\alpha$ & 0 & -537 & & \\
\hline & $\zeta_{4 p}$ & 11232 & 11776 & 1.048 & \\
\hline & $\zeta_{5 p}$ & 2920 & 2920 & 1.000 & $\mathrm{~F}$ \\
\hline & $\mathrm{F}^{2}(4 \mathrm{p}, 5 \mathrm{p})$ & 29125 & 29164 & 1.001 & \\
\hline & $G^{0}(4 p, 5 p)$ & 6338 & 6086 & 0.960 & \\
\hline & $G^{2}(4 p, 5 p)$ & 8477 & 5272 & 0.622 & \\
\hline \multicolumn{6}{|c|}{ Odd parity } \\
\hline \multirow[t]{3}{*}{$4 s 4 p^{5}$} & $E_{\mathrm{av}}$ & 246126 & 238581 & & \\
\hline & $\zeta_{4 p}$ & 10648 & 11005 & 1.034 & \\
\hline & $\mathrm{G}^{1}(4 \mathrm{~s}, 4 \mathrm{p})$ & 112472 & 98647 & 0.877 & \\
\hline \multirow[t]{8}{*}{$4 p^{3} 4 d$} & $E_{\mathrm{av}}$ & 320698 & 319713 & & \\
\hline & $\mathrm{F}^{2}(4 \mathrm{p}, 4 \mathrm{p})$ & 84870 & 81614 & 0.962 & \\
\hline & $\alpha$ & 0 & -508 & & \\
\hline & $\zeta_{4 p}$ & 10822 & 11010 & 1.017 & \\
\hline & $\zeta_{4 d}$ & 824 & 795 & 0.964 & \\
\hline & $\mathrm{F}^{2}(4 \mathrm{p}, 4 \mathrm{~d})$ & 73259 & 69858 & 0.954 & \\
\hline & $\mathrm{G}^{1}(4 \mathrm{p}, 4 \mathrm{~d})$ & 91609 & 77513 & 0.846 & \\
\hline & $\mathrm{G}^{3}(4 \mathrm{p}, 4 \mathrm{~d})$ & 57095 & 48489 & 0.849 & \\
\hline \multirow[t]{5}{*}{$4 p^{3} 5 s$} & $E_{\mathrm{av}}$ & 448971 & 447229 & & \\
\hline & $\mathrm{F}^{2}(4 \mathrm{p}, 4 \mathrm{p})$ & 85823 & 80727 & 0.941 & \\
\hline & $\alpha$ & 0 & -667 & & \\
\hline & $\zeta_{4 p}$ & 11148 & 11790 & 1.058 & \\
\hline & $\mathrm{G}^{1}(4 \mathrm{p}, 5 \mathrm{~s})$ & 9475 & 8104 & 0.855 & \\
\hline $4 s 4 p^{5}-4 p^{3} 4 d$ & $\mathrm{R}^{1}(4 \mathrm{p} 4 \mathrm{p} ; 4 \mathrm{~s} 4 \mathrm{~d})$ & 100074 & 78158 & 0.781 & \\
\hline
\end{tabular}

Notes. ${ }^{(a)}$ F: Fixed parameter value. 
T. Rauch et al.: Stellar laboratories. VIII.

Table A.5. Comparison between available experimental and calculated energy levels in Zr IV.

\begin{tabular}{|c|c|c|c|c|}
\hline$E_{\text {exp }}{ }^{a}$ & $E_{\text {calc }}^{b}$ & $\Delta E$ & $J$ & Leading components (in \%) in $L S$ coupling $^{c}$ \\
\hline \multicolumn{5}{|c|}{ Even parity } \\
\hline 0.00 & 0000 & 0 & 1.5 & $994 d^{2} \mathrm{D}$ \\
\hline 1250.70 & 1251 & 0 & 2.5 & $994 d^{2} \mathrm{D}$ \\
\hline 38258.35 & 38258 & 0 & 0.5 & $995 s^{2} S$ \\
\hline 146652.40 & 146652 & 0 & 1.5 & $1005 d^{2} \mathrm{D}$ \\
\hline 147002.46 & 147002 & 0 & 2.5 & $1005 d^{2} \mathrm{D}$ \\
\hline 152513.00 & 152513 & 0 & 0.5 & $1006 s^{2} S$ \\
\hline 197765.10 & 197765 & 0 & 1.5 & $1006 d^{2} \mathrm{D}$ \\
\hline 197930.43 & 197930 & 0 & 2.5 & $1006 d^{2} \mathrm{D}$ \\
\hline 200123.69 & 200124 & 0 & 0.5 & $1007 \mathrm{~s}^{2} \mathrm{~S}$ \\
\hline 206864.42 & 206863 & 0 & 3.5 & $1005 \mathrm{~g}^{2} \mathrm{G}$ \\
\hline 206864.68 & 206866 & -1 & 4.5 & $1005 \mathrm{~g}^{2} \mathrm{G}$ \\
\hline 224813.48 & 224813 & 0 & 0.5 & $1008 s^{2} s$ \\
\hline 228479.86 & 228479 & 0 & 3.5 & $1006 \mathrm{~g}^{2} \mathrm{G}$ \\
\hline 228480.08 & 228480 & 0 & 4.5 & $1006 \mathrm{~g}^{2} \mathrm{G}$ \\
\hline 241526.36 & 241526 & 0 & 3.5 & $1007 \mathrm{~g}^{2} \mathrm{G}$ \\
\hline 241526.52 & 241527 & 0 & 4.5 & $1007 \mathrm{~g}^{2} \mathrm{G}$ \\
\hline 249995.33 & 249995 & 0 & 3.5 & $1008 g^{2} \mathrm{G}$ \\
\hline 249995.44 & 249996 & 0 & 4.5 & $1008 \mathrm{~g}^{2} \mathrm{G}$ \\
\hline 255800.20 & 255801 & -1 & 3.5 & $1009 \mathrm{~g}^{2} \mathrm{G}$ \\
\hline 255801.50 & 255801 & 1 & 4.5 & $1009 \mathrm{~g}^{2} \mathrm{G}$ \\
\hline \multicolumn{5}{|c|}{ Odd parity } \\
\hline 81976.50 & 81976 & 0 & 0.5 & $995 \mathrm{p}^{2} \mathrm{P}$ \\
\hline 84461.35 & 84461 & 0 & 1.5 & $995 \mathrm{p}^{2} \mathrm{P}$ \\
\hline 159066.75 & 159041 & 26 & 2.5 & $984 \mathrm{f}^{2} \mathrm{~F}$ \\
\hline 159086.91 & 159112 & -25 & 3.5 & $984 \mathrm{f}^{2} \mathrm{~F}$ \\
\hline 169809.71 & 169810 & 0 & 0.5 & $1006 p^{2} P$ \\
\hline 170815.11 & 170815 & 0 & 1.5 & $1006 \mathrm{p}^{2} \mathrm{P}$ \\
\hline 201114.14 & 201105 & 9 & 2.5 & $975 f^{2} \mathrm{~F}$ \\
\hline 201162.65 & 201171 & -9 & 3.5 & $975 f^{2} F$ \\
\hline 208783.36 & 208783 & 0 & 0.5 & $1007 p^{2} \mathrm{P}$ \\
\hline 209297.66 & 209298 & 0 & 1.5 & $1007 p^{2} P$ \\
\hline 224419.90 & 224425 & -5 & 2.5 & $966 \mathrm{f}^{2} \mathrm{~F}$ \\
\hline 224488.11 & 224483 & 5 & 3.5 & $976 f^{2} \mathrm{~F}$ \\
\hline 228743.87 & 228744 & 0 & 4.5 & $1006 \mathrm{~h}^{2} \mathrm{H}$ \\
\hline 228743.87 & 228744 & 0 & 5.5 & $1006 h^{2} \mathrm{H}$ \\
\hline
\end{tabular}

Notes. Energies are given in $\mathrm{cm}^{-1} .{ }^{(a)}$ From Reader \& Acquista (1997). ${ }^{(b)}$ This work. 
Table A.6. Comparison between available experimental and calculated energy levels in Zr v.

\begin{tabular}{|c|c|c|c|c|}
\hline$E_{\exp }{ }^{a}$ & $E_{\text {calc }^{b}}^{b}$ & $\Delta E$ & $J$ & Leading components (in \%) in $L S$ coupling ${ }^{c}$ \\
\hline \multicolumn{5}{|c|}{ Even parity } \\
\hline 0.00 & 0 & 0 & 0 & $974 \mathrm{p}^{6}{ }^{1} \mathrm{~S}$ \\
\hline 371895.16 & 372099 & -204 & 1 & $844 p^{5} 5 p^{3} S+134 p^{5} 5 p^{3} P$ \\
\hline 376897.68 & 376807 & 91 & 2 & $574 p^{5} 5 p^{3} D+364 p^{5} 5{ }^{1} D+74 p^{5} 5 p^{3} P$ \\
\hline 378753.36 & 378653 & 100 & 3 & $994 p^{5} 5 p^{3} D$ \\
\hline 380855.53 & 380904 & -48 & 1 & $464 p^{5} 5 p^{1} P+304 p^{5} 5 p^{3} D+204 p^{5} 5 p^{3} P$ \\
\hline 382985.08 & 382952 & 33 & 2 & $674 p^{5} 5 p^{3} P+304 p^{5} 5 p^{1} D$ \\
\hline 388852.95 & 388865 & -12 & 0 & $774 p^{5} 5 p^{3} P+224 p^{5} 5 p^{1} S$ \\
\hline 391998.41 & 392073 & -75 & 1 & $644 p^{5} 5 p^{3} D+334 p^{5} 5 p^{1} P$ \\
\hline 395994.98 & 395944 & 51 & 2 & $404 p^{5} 5 p^{3} D+334 p^{5} 5 p^{1} D+254 p^{5} 5 p^{3} P$ \\
\hline 396300.35 & 396396 & -96 & 1 & $644 p^{5} 5 p^{3} P+194 p^{5} 5 p^{1} P+114 p^{5} 5 p^{3} S$ \\
\hline 402688.40 & 402529 & 160 & 0 & $764 p^{5} 5 p^{1} S+224 p^{5} 5 p^{3} P$ \\
\hline 434714.60 & 434703 & 12 & 1 & $554 \mathrm{~s} 4 \mathrm{p}^{6} 4 \mathrm{~d}^{3} \mathrm{D}+314 \mathrm{p}^{5} 4 \mathrm{f}^{3} \mathrm{D}+84 \mathrm{p}^{4} 4 \mathrm{~d}^{2}{ }^{3} \mathrm{D}$ \\
\hline 435759.10 & 435755 & 4 & 2 & $564 s 4 p^{6} 4 d^{3} D+294 p^{5} 4 f^{3} D+84 p^{4} 4 d^{2}{ }^{3} D$ \\
\hline 437678.10 & 437641 & 38 & 3 & $584 \mathrm{~s} 4 \mathrm{p}^{6} 4 \mathrm{~d}^{3} \mathrm{D}+254 \mathrm{p}^{5} 4 \mathrm{f}^{3} \mathrm{D}+94 \mathrm{p}^{4} 4 \mathrm{~d}^{2}{ }^{3} \mathrm{D}$ \\
\hline 450133.70 & 450156 & -22 & 2 & $494 s 4 p^{6} 4 d^{1} D+194 p^{4} 4 d^{2}{ }^{1} D+184 p^{5} 4 f^{1} D$ \\
\hline 453680.80 & 453610 & 71 & 5 & $944 \mathrm{p}^{5} 4 \mathrm{f}^{3} \mathrm{G}$ \\
\hline 454538.80 & 454537 & 1 & 4 & $594 p^{5} 4 f^{3} G+334 p^{5} 4 f^{1} G$ \\
\hline 457546.70 & 457482 & 65 & 3 & $434 p^{5} 4 f^{3} G+294 p^{5} 4 f^{1} F+224 p^{5} 4 f^{3} F$ \\
\hline 458432.20 & 458479 & -47 & 4 & $544 p^{5} 4 f^{3} F+314 p^{5} 4 f^{1} G+84 p^{5} 4 f^{3} G$ \\
\hline 460476.90 & 460554 & -77 & 1 & $624 p^{5} 4 f^{3} D+184 s 4 p^{6} 4 d^{3} D+104 p^{4} 4 d^{2}{ }^{3} D$ \\
\hline 460694.10 & 460714 & -20 & 2 & $424 p^{5} 4 f^{3} D+274 p^{5} 4 f^{3} F+144 s 4 p^{6} 4 d^{3} D$ \\
\hline 460767.50 & 460886 & -119 & 3 & $284 p^{5} 4 f^{3} D+274 p^{5} 4 f^{3} F+214 p^{5} 4 f^{1} F$ \\
\hline 464015.40 & 463932 & 83 & 2 & $324 p^{5} 4 f^{3} F+314 p^{5} 4 f^{1} D+154 s 4 p^{6} 4 d^{1} D$ \\
\hline 470773.50 & 470677 & 96 & 3 & $504 p^{5} 4 f^{3} G+254 p^{5} 4 f^{1} F+184 p^{5} 4 f^{3} F$ \\
\hline 471762.40 & 471785 & -22 & 4 & $374 p^{5} 4 f^{3} F+304 p^{5} 4 f^{1} G+264 p^{5} 4 f^{3} G$ \\
\hline 473715.40 & 473766 & -51 & 3 & $404 p^{5} 4 f^{3} D+264 p^{5} 4 f^{3} F+194 p^{5} 4 f^{1} F$ \\
\hline 476130.20 & 476166 & -35 & 2 & $464 p^{5} 4 f^{1} D+314 p^{5} 4 f^{3} F+114 p^{5} 4 f^{3} D$ \\
\hline 491116.00 & 491414 & -298 & 1 & $784 p^{5} 6 p^{3} S+164 p^{5} 6 p^{3} P$ \\
\hline 494472.00 & 495996 & -1524 & 1 & $554 p^{5} 6 p^{1} P+224 p^{5} 6 p^{3} P+214 p^{5} 6 p^{3} D$ \\
\hline 494760.00 & 494729 & 31 & 3 & $994 p^{5} 6 p^{3} D$ \\
\hline 495912.00 & 494141 & 1771 & 2 & $524 p^{5} 6 p^{3} D+414 p^{5} 6 p{ }^{1} D+64 p^{5} 6 p^{3} p$ \\
\hline 496428.00 & 496722 & -294 & 2 & $734 p^{5} 6 p^{3} P+244 p^{5} 6 p^{1} D$ \\
\hline 499459.00 & 498891 & 568 & 0 & $554 p^{5} 6 p^{1} S+424 p^{5} 6 p^{3} P$ \\
\hline 509310.00 & 509042 & 268 & 1 & $674 p^{5} 6 p^{3} D+304 p^{5} 6 p^{1} P$ \\
\hline 510066.00 & 510179 & -113 & 1 & $604 p^{5} 6 p^{3} P+134 p^{5} 6 p^{3} s+124 p^{5} 6 p^{1} P$ \\
\hline 510942.00 & 511814 & -872 & 0 & $574 p^{5} 6 p^{3} P+384 p^{5} 6 p^{1} S$ \\
\hline 511263.00 & 510586 & 677 & 2 & $\begin{array}{l}454 p^{5} 6 p^{3} D+334 p^{5} 6 p^{1} D+214 p^{5} 6 p^{3} P \\
\text { dd parity }\end{array}$ \\
\hline 241381.30 & 241649 & -268 & 0 & $994 p^{5} 4 d^{3} P$ \\
\hline 243560.80 & 243779 & -218 & 1 & $974 p^{5} 4 d^{3} \mathrm{P}$ \\
\hline 247962.30 & 248100 & -138 & 2 & $914 p^{5} 4 d^{3} P+64 p^{5} 4 d^{3} D$ \\
\hline 251283.30 & 250854 & 429 & 4 & $994 p^{5} 4 d^{3} F$ \\
\hline 253753.40 & 253327 & 426 & 3 & $874 p^{5} 4 d^{3} F+84 p^{5} 4 d^{1} F+54 p^{5} 4 d^{3} D$ \\
\hline 257361.30 & 257118 & 243 & 2 & $754 p^{5} 4 d^{3} F+144 p^{5} 4 d^{1} D+104 p^{5} 4 d^{3} D$ \\
\hline 265845.50 & 266213 & -367 & 3 & $654 p^{5} 4 d^{3} \mathrm{D}+354 \mathrm{p}^{5} 4 \mathrm{~d}^{1} \mathrm{~F}$ \\
\hline 270560.80 & 270736 & -176 & 2 & $494 p^{5} 4 d^{1} D+264 p^{5} 4 d^{3} D+244 p^{5} 4 d^{3} F$ \\
\hline 271601.60 & 271544 & 57 & 1 & $964 p^{5} 4 d^{3} D$ \\
\hline 274654.60 & 274810 & -155 & 2 & $574 p^{5} 4 d^{3} D+344 p^{5} 4 d^{1} D+84 p^{5} 4 d^{3} P$ \\
\hline 277145.50 & 276979 & 166 & 3 & $574 p^{5} 4 d^{1} F+304 p^{5} 4 d^{3} D+134 p^{5} 4 d^{3} F$ \\
\hline 325014.87 & 325066 & -52 & 2 & $994 p^{5} 5 s^{3} \mathrm{P}$ \\
\hline 327616.99 & 327532 & 85 & 1 & $384 p^{5} 5 s^{1} P+344 p^{5} 5 s^{3} P+254 p^{5} 4 d^{1} P$ \\
\hline 328940.75 & 328971 & -30 & 1 & $684 p^{5} 4 d^{1} P+154 p^{5} 5 s^{3} P+124 p^{5} 5 s^{1} P$ \\
\hline 340315.49 & 340258 & 57 & 0 & $994 \mathrm{p}^{5} 5 \mathrm{~s}^{3} \mathrm{P}$ \\
\hline 342245.65 & 342305 & -60 & 1 & $504 p^{5} 5 s^{3} P+494 p^{5} 5 s^{1} P$ \\
\hline 452938.91 & 452953 & -14 & 0 & $994 p^{5} 5 d^{3} P$ \\
\hline
\end{tabular}

Notes. Energies are given in $\mathrm{cm}^{-1}$. ${ }^{(a)}$ From Reader \& Acquista (1979) and Khan et al. (1981). ${ }^{(b)}$ This work. ${ }^{(c)}$ Only the first three components that are larger than $5 \%$ are given. 
T. Rauch et al.: Stellar laboratories. VIII.

Table A.6. continued.

\begin{tabular}{|c|c|c|c|c|}
\hline$E_{\exp }{ }^{a}$ & $E_{\text {calc }}^{b}$ & $\Delta E$ & $J$ & Leading components (in \%) in $L S$ coupling $^{c}$ \\
\hline 453905.60 & 453911 & -5 & 1 & $894 p^{5} 5 d^{3} P+104 p^{5} 5 d^{3} D$ \\
\hline 455444.40 & 455398 & 47 & 4 & $994 p^{5} 5 d^{3} \mathrm{~F}$ \\
\hline 455630.80 & 455629 & 2 & 2 & $664 p^{5} 5 d^{3} P+244 p^{5} 5 d^{3} D+94 p^{5} 5 d^{1} D$ \\
\hline 455925.27 & 455941 & -16 & 3 & $604 p^{5} 5 d^{3} F+344 p^{5} 5 d^{1} F+54 p^{5} 5 d^{3} D$ \\
\hline 457613.10 & 457595 & 18 & 2 & $444 p^{5} 5 d^{1} D+314 p^{5} 5 d^{3} F+234 p^{5} 5 d^{3} D$ \\
\hline 458523.70 & 458496 & 28 & 3 & $664 p^{5} 5 d^{3} D+304 p^{5} 5 d^{1} F$ \\
\hline 462307.40 & 462375 & -68 & 1 & $564 p^{5} 5 d^{3} D+374 p^{5} 5 d^{1} P$ \\
\hline 471306.30 & 471306 & 0 & 2 & $664 p^{5} 5 d^{3} F+254 p^{5} 5 d^{1} D+74 p^{5} 5 d^{3} D$ \\
\hline 472015.28 & 472047 & -31 & 2 & $414 p^{5} 5 d^{3} D+284 p^{5} 5 d^{3} P+184 p^{5} 5 d^{1} D$ \\
\hline 472338.00 & 472335 & 3 & 2 & $894 p^{5} 6 s^{3} \mathrm{P}$ \\
\hline 472520.00 & 472529 & -9 & 3 & $364 p^{5} 5 d^{3} F+354 p^{5} 5 d^{1} F+284 p^{5} 5 d^{3} D$ \\
\hline 473172.70 & 473173 & -1 & 1 & $614 \mathrm{p}^{5} 6 \mathrm{~s}^{1} \mathrm{P}+364 \mathrm{p}^{5} 6 \mathrm{~s}^{3} \mathrm{P}$ \\
\hline 476477.40 & 476432 & 45 & 1 & $564 p^{5} 5 d^{1} P+324 p^{5} 5 d^{3} D+64 p^{5} 5 d^{3} P$ \\
\hline 487746.60 & 487747 & 0 & 0 & $1004 p^{5} 6 s^{3} \mathrm{P}$ \\
\hline 488292.70 & 488292 & 0 & 1 & $624 p^{5} 6 s^{3} P+384 p^{5} 6 s^{1} P$ \\
\hline 528422.80 & 528711 & -288 & 1 & $834 p^{5} 6 d^{3} P+154 p^{5} 6 d^{3} D$ \\
\hline 529161.60 & 529325 & -163 & 4 & $1004 p^{5} 6 d^{3} F$ \\
\hline 529283.30 & 529342 & -59 & 2 & $544 p^{5} 6 d^{3} P+334 p^{5} 6 d^{3} D+114 p^{5} 6 d^{1} D$ \\
\hline 529299.60 & 529363 & -63 & 3 & $524 p^{5} 6 d^{3} F+444 p^{5} 6 d^{1} F$ \\
\hline 530119.70 & 529936 & 183 & 2 & $514 p^{5} 6 d^{1} D+244 p^{5} 6 d^{3} F+234 p^{5} 6 d^{3} D$ \\
\hline 530465.50 & 530165 & 300 & 3 & $724 p^{5} 6 d^{3} D+224 p^{5} 6 d^{1} F+54 p^{5} 6 d^{3} F$ \\
\hline 531839.00 & 531753 & 86 & 1 & $564 p^{5} 6{ }^{1} P+394 p^{5} 6 d^{3} D$ \\
\hline 536682.20 & 536674 & 8 & 2 & $1004 p^{5} 5 g^{3} \mathrm{~F}$ \\
\hline 536731.50 & 536723 & 9 & 3 & $604 p^{5} 5 g^{3} F+394 p^{5} 5 g{ }^{1} F$ \\
\hline 536763.90 & 536761 & 3 & 2 & $1004 \mathrm{p}^{5} 7 \mathrm{~s}^{3} \mathrm{P}$ \\
\hline 536961.40 & 536976 & -14 & 6 & $1004 p^{5} 5 g^{3} \mathrm{H}$ \\
\hline 536983.90 & 536996 & -12 & 5 & $534 p^{5} 5 g{ }^{1} \mathrm{H}+464 \mathrm{p}^{5} 5 \mathrm{~g}{ }^{3} \mathrm{H}$ \\
\hline 537213.40 & 537217 & -4 & 1 & $644 p^{5} 7 \mathrm{~s}{ }^{1} \mathrm{P}+354 \mathrm{p}^{5} 7 \mathrm{~s}^{3} \mathrm{P}$ \\
\hline 537501.90 & 537499 & 3 & 4 & $464 p^{5} 5 g{ }^{3} F+304 p^{5} 5 g{ }^{3} G+244 p^{5} 5 g{ }^{1} G$ \\
\hline 537539.20 & 537528 & 11 & 3 & $544 p^{5} 5 g^{3} \mathrm{G}+294 \mathrm{p}^{5} 5 \mathrm{~g}{ }^{1} \mathrm{~F}+174 \mathrm{p}^{5} 5 \mathrm{~g}^{3} \mathrm{~F}$ \\
\hline 537806.70 & 537807 & -1 & 4 & $394 p^{5} 5 g^{1} G+314 p^{5} 5 g{ }^{3} G+304 p^{5} 5 g^{3} H$ \\
\hline 537816.50 & 537820 & -3 & 5 & $704 p^{5} 5 g^{3} \mathrm{G}+154 p^{5} 5 g^{3} \mathrm{H}+144 p^{5} 5 g^{1} \mathrm{H}$ \\
\hline 546323.00 & 546325 & -2 & 1 & $464 p^{5} 6 d^{3} D+414 p^{5} 6 d^{1} P+124 p^{5} 6 d^{3} P$ \\
\hline 552258.20 & 552265 & -7 & 0 & $1004 p^{5} 7 s^{3} \mathrm{P}$ \\
\hline 552521.10 & 552515 & 6 & 1 & $644 \mathrm{p}^{5} 7 \mathrm{~s}^{3} \mathrm{P}+354 \mathrm{p}^{5} 7 \mathrm{~s}{ }^{1} \mathrm{P}$ \\
\hline 552878.20 & 552884 & -6 & 4 & $664 p^{5} 5 g^{3} \mathrm{H}+264 p^{5} 5 g^{1} G+54 p^{5} 5 g^{3} G$ \\
\hline 552894.50 & 552889 & 5 & 4 & $504 p^{5} 5 g^{3} F+344 p^{5} 5 g^{3} G+114 p^{5} 5 g^{1} G$ \\
\hline 552894.70 & 552905 & -10 & 5 & $384 p^{5} 5 g^{3} \mathrm{H}+324 p^{5} 5 \mathrm{~g}{ }^{1} \mathrm{H}+304 p^{5} 5 \mathrm{~g}^{3} \mathrm{G}$ \\
\hline 552933.50 & 552923 & 11 & 3 & $464 p^{5} 5 g^{3} \mathrm{G}+314 \mathrm{p}^{5} 5 \mathrm{~g}^{1} \mathrm{~F}+234 \mathrm{p}^{5} 5 \mathrm{~g}^{3} \mathrm{~F}$ \\
\hline 568040.00 & 567226 & 814 & 1 & $744 p^{5} 7 d^{3} P+104 p^{5} 7 d^{3} D+104 s 4 p^{6} 5 p^{3} P$ \\
\hline 570779.30 & 570772 & 7 & 2 & $1004 p^{5} 6 g^{3} \mathrm{~F}$ \\
\hline 570828.20 & 570823 & 5 & 3 & $634 p^{5} 6 g{ }^{3} F+374 p^{5} 6 g{ }^{1} F$ \\
\hline 570946.50 & 570957 & -11 & 6 & $1004 p^{5} 6 g{ }^{3} \mathrm{H}$ \\
\hline 570967.60 & 570977 & -9 & 5 & $534 p^{5} 6 g^{1} \mathrm{H}+474 p^{5} 6 g^{3} \mathrm{H}$ \\
\hline 571271.70 & 571267 & 4 & 4 & $444 p^{5} 6 g{ }^{3} F+314 p^{5} 6 g^{3} G+254 p^{5} 6 g^{1} G$ \\
\hline 571306.30 & 571301 & 5 & 3 & $554 p^{5} 6 g^{3} \mathrm{G}+314 \mathrm{p}^{5} 6 \mathrm{~g}{ }^{1} \mathrm{~F}+134 \mathrm{p}^{5} 6 \mathrm{~g}^{3} \mathrm{~F}$ \\
\hline 571376.00 & 571674 & -298 & 1 & $644 \mathrm{p}^{5} 8 \mathrm{~s}^{1} \mathrm{P}+344 \mathrm{p}^{5} 8 \mathrm{~s}^{3} \mathrm{P}$ \\
\hline 571443.60 & 571444 & 0 & 4 & $404 p^{5} 6 g{ }^{1} G+324 p^{5} 6{ }^{3} G+284 p^{5} 6 g{ }^{3} H$ \\
\hline 571452.20 & 571454 & -2 & 5 & $714 p^{5} 6 g^{3} \mathrm{G}+144 p^{5} 6{ }^{3} \mathrm{H}+144 \mathrm{p}^{5} 6 \mathrm{~g}{ }^{1} \mathrm{H}$ \\
\hline 573776.00 & 573860 & -84 & 1 & $594 s 4 p^{6} 5 p^{3} P+194 p^{4} 4 d 5 p^{3} P+94 p^{5} 7 d^{3} P$ \\
\hline 583420.00 & 584144 & -724 & 1 & $444 p^{5} 7 d^{3} D+374 p^{5} 7 d^{1} P+144 p^{5} 7 d^{3} P$ \\
\hline 586704.90 & 586704 & 0 & 4 & $554 p^{5} 6 g^{3} F+224 p^{5} 6 g^{3} G+224 p^{5} 6 g^{1} G$ \\
\hline 586718.20 & 586718 & 0 & 4 & $714 p^{5} 6 g^{3} \mathrm{H}+154 p^{5} 6{ }^{3} \mathrm{G}+134 p^{5} 6 \mathrm{~g}^{1} \mathrm{G}$ \\
\hline 586734.50 & 586735 & -1 & 5 & $394 p^{5} 6 g^{3} \mathrm{H}+334 p^{5} 6 \mathrm{~g}{ }^{1} \mathrm{H}+284 \mathrm{p}^{5} 6 \mathrm{~g}^{3} \mathrm{G}$ \\
\hline 586882.00 & 586588 & 294 & 1 & $654 \mathrm{p}^{5} 8 \mathrm{~s}^{3} \mathrm{P}+344 \mathrm{p}^{5} 8 \mathrm{~s}^{1} \mathrm{P}$ \\
\hline 591916.00 & 591916 & 0 & 1 & $664 p^{5} 9 s^{1} P+344 p^{5} 9 s^{3} P$ \\
\hline 605118.00 & 605118 & 0 & 1 & $664 \mathrm{p}^{5} 10 \mathrm{~s}{ }^{1} \mathrm{P}+344 \mathrm{p}^{5} 10 \mathrm{~s}{ }^{3} \mathrm{P}$ \\
\hline
\end{tabular}


Table A.7. Comparison between available experimental and calculated energy levels in Zr VI.

\begin{tabular}{|c|c|c|c|c|}
\hline$E_{\text {exp }}^{a}$ & $E_{\text {calc }}^{b}$ & $\Delta E$ & $J$ & Leading components (in \%) in $L S$ coupling ${ }^{c}$ \\
\hline & & & & Odd parity \\
\hline 0.00 & 0 & 0 & 1.5 & $974 \mathrm{p}^{52} \mathrm{P}$ \\
\hline 15602.78 & 15603 & 0 & 0.5 & $974 \mathrm{p}^{52} \mathrm{P}$ \\
\hline 421257.96 & 421364 & -106 & 1.5 & $624 p^{4}\left({ }^{3} P\right) 5 p{ }^{4} P+94 p^{4}\left({ }^{3} P\right) 5 p{ }^{4} S+94 p^{4}\left({ }^{1} D\right) 5 p{ }^{2} P$ \\
\hline 421991.19 & 421898 & 93 & 2.5 & $684 p^{4}\left({ }^{3} P\right) 5 p{ }^{4} P+234 p^{4}\left({ }^{3} P\right) 5 p{ }^{4} D$ \\
\hline 425678.16 & 426017 & -339 & 0.5 & $234 p^{4}\left({ }^{3} P\right) 5 p^{2} P+444 p^{4}\left({ }^{3} P\right) 5 p{ }^{4} P+194 p^{4}\left({ }^{1} D\right) 5 p{ }^{2} P$ \\
\hline 427118.65 & 427134 & -15 & 2.5 & $604 p^{4}\left({ }^{3} P\right) 5 p^{2} D+144 p^{4}\left({ }^{3} P\right) 5 p{ }^{4} P+134 p^{4}\left({ }^{3} P\right) 5 p{ }^{4} D$ \\
\hline 427649.11 & 427421 & 228 & 3.5 & $894 p^{4}\left({ }^{3} \mathrm{P}\right) 5 \mathrm{p}^{4} \mathrm{D}+104 \mathrm{p}^{4}\left({ }^{1} \mathrm{D}\right) 5 \mathrm{p}^{2} \mathrm{~F}$ \\
\hline 434797.76 & 434744 & 53 & 0.5 & $394 p^{4}\left({ }^{3} P\right) 5 p{ }^{4} P+224 p^{4}\left({ }^{3} P\right) 5 p{ }^{4} D+184 p^{4}\left({ }^{3} P\right) 5 p{ }^{2} P$ \\
\hline 435427.69 & 435124 & 304 & 1.5 & $334 p^{4}\left({ }^{3} P\right) 5 p^{4} D+184 p^{4}\left({ }^{3} P\right) 5 p^{2} P+224 p^{4}\left({ }^{3} P\right) 5 p{ }^{2} D$ \\
\hline 436859.11 & 436770 & 89 & 0.5 & $604 p^{4}\left({ }^{3} P\right) 5 p{ }^{4} D+144 p^{4}\left({ }^{3} P\right) 5 p^{2} S+134 p^{4}\left({ }^{3} P\right) 5 p{ }^{4} P$ \\
\hline 437477.01 & 437605 & -128 & 1.5 & $484 p^{4}\left({ }^{3} \mathrm{P}\right) 5 \mathrm{p}{ }^{4} \mathrm{D}+324 \mathrm{p}^{4}\left({ }^{3} \mathrm{P}\right) 5 \mathrm{p}{ }^{2} \mathrm{P}+104 \mathrm{p}^{4}\left({ }^{1} \mathrm{D}\right) 5 \mathrm{p}{ }^{2} \mathrm{P}$ \\
\hline 440554.88 & 440364 & 191 & 2.5 & $594 p^{4}\left({ }^{3} P\right) 5 p{ }^{4} D+254 p^{4}\left({ }^{3} P\right) 5 p^{2} D+134 p^{4}\left({ }^{3} P\right) 5 p{ }^{4} P$ \\
\hline 442453.66 & 442488 & -34 & 1.5 & $284 p^{4}\left({ }^{3} \mathrm{P}\right) 5 \mathrm{p}^{2} \mathrm{D}+244 \mathrm{p}^{4}\left({ }^{3} \mathrm{P}\right) 5 \mathrm{p}{ }^{4} \mathrm{~S}+154 \mathrm{p}^{4}\left({ }^{3} \mathrm{P}\right) 5 \mathrm{p}{ }^{4} \mathrm{P}$ \\
\hline 444340.07 & 444700 & -360 & 0.5 & $674 p^{4}\left({ }^{3} P\right) 5 p^{2} S+134 p^{4}\left({ }^{3} P\right) 5 p^{2} P+104 p^{4}\left({ }^{3} P\right) 5 p^{4} D$ \\
\hline 444879.34 & 444961 & -82 & 1.5 & $454 p^{4}\left({ }^{3} P\right) 5 p{ }^{4} S+424 p^{4}\left({ }^{3} P\right) 5 p^{2} D+54 p^{4}\left({ }^{3} P\right) 5 p{ }^{4} P$ \\
\hline 449730.72 & 449653 & 77 & 2.5 & $834 p^{4}\left({ }^{1} D\right) 5 p{ }^{2} F+84 p^{4}\left({ }^{3} P\right) 5 p{ }^{2} D$ \\
\hline 452999.87 & 452910 & 90 & 3.5 & $884 p^{4}\left({ }^{1} \mathrm{D}\right) 5 \mathrm{p}^{2} \mathrm{~F}+104 \mathrm{p}^{4}\left({ }^{3} \mathrm{P}\right) 5 \mathrm{p}^{4} \mathrm{D}$ \\
\hline 455878.16 & 455971 & -92 & 1.5 & $574 p^{4}\left({ }^{1} \mathrm{D}\right) 5 \mathrm{p}^{2} \mathrm{P}+214 \mathrm{p}^{4}\left({ }^{1} \mathrm{D}\right) 5 \mathrm{p}^{2} \mathrm{D}+94 \mathrm{p}^{4}\left({ }^{3} \mathrm{P}\right) 5 \mathrm{p}^{2} \mathrm{P}$ \\
\hline 459077.64 & 459024 & 54 & 1.5 & $704 p^{4}\left({ }^{1} \mathrm{D}\right) 5 \mathrm{p}^{2} \mathrm{D}+194 \mathrm{p}^{4}\left({ }^{3} \mathrm{P}\right) 5 \mathrm{p}{ }^{2} \mathrm{P}+84 \mathrm{p}^{4}\left({ }^{1} \mathrm{D}\right) 5 \mathrm{p}{ }^{2} \mathrm{P}$ \\
\hline 459580.77 & 459640 & -60 & 2.5 & $894 p^{4}\left({ }^{1} D\right) 5 p^{2} D$ \\
\hline 464724.05 & 464719 & 5 & 0.5 & $614 p^{4}\left({ }^{1} \mathrm{D}\right) 5 \mathrm{p}^{2} \mathrm{P}+344 \mathrm{p}^{4}\left({ }^{3} \mathrm{P}\right) 5 \mathrm{p}{ }^{2} \mathrm{P}$ \\
\hline 482699.28 & 482631 & 68 & 0.5 & $784 p^{4}\left({ }^{1} S\right) 5 p{ }^{2} P+94 p^{4}\left({ }^{3} P\right) 5 p{ }^{2} P+74 p^{4}\left({ }^{3} P\right) 5 p{ }^{4} D$ \\
\hline 484897.26 & 484977 & -80 & 1.5 & $\begin{array}{l}414 p^{4}\left({ }^{1} S\right) 5 p^{2} P+294 s 4 p^{5} 4 d^{2} D+84 p^{4}\left({ }^{1} D\right) 4 f^{2} D \\
\text { Even parity }\end{array}$ \\
\hline 191570.67 & 191601 & -30 & 0.5 & $794 s 4 p^{6}{ }^{2} S+214 p^{4}\left({ }^{1} D\right) 4 d^{2} S$ \\
\hline 248940.11 & 248835 & 105 & 2.5 & $884 p^{4}\left({ }^{3} P\right) 4 d^{4} D$ \\
\hline 249322.89 & 249299 & 24 & 3.5 & $904 p^{4}\left({ }^{3} P\right) 4 d{ }^{4} D+64 p^{4}\left({ }^{3} P\right) 4 d{ }^{4} F$ \\
\hline 250017.63 & 249918 & 99 & 1.5 & $854 p^{4}\left({ }^{3} P\right) 4 d^{4} D$ \\
\hline 251818.70 & 251917 & -98 & 0.5 & $854 p^{4}\left({ }^{3} \mathrm{P}\right) 4 d^{4} \mathrm{D}+64 \mathrm{p}^{4}\left({ }^{1} \mathrm{D}\right) 4 \mathrm{~d}^{2} \mathrm{P}+54 \mathrm{p}^{4}\left({ }^{3} \mathrm{P}\right) 4 \mathrm{~d}^{2} \mathrm{P}$ \\
\hline 261642.90 & 261178 & 465 & 4.5 & $894 \mathrm{p}^{4}\left({ }^{3} \mathrm{P}\right) 4 \mathrm{~d}^{4} \mathrm{~F}+104 \mathrm{p}^{4}\left({ }^{1} \mathrm{D}\right) 4 \mathrm{~d}^{2} \mathrm{G}$ \\
\hline 266145.41 & 265622 & 523 & 3.5 & $654 p^{4}\left({ }^{3} P\right) 4 d^{4} F+174 p^{4}\left({ }^{3} P\right) 4 d^{2} F+134 p^{4}\left({ }^{1} D\right) 4 d^{2} G$ \\
\hline 266278.49 & 267703 & -1.425 & 0.5 & $434 p^{4}\left({ }^{1} \mathrm{D}\right) 4 d^{2} \mathrm{P}+374 \mathrm{p}^{4}\left({ }^{3} \mathrm{P}\right) 4 \mathrm{~d}^{2} \mathrm{P}+144 \mathrm{p}^{4}\left({ }^{3} \mathrm{P}\right) 4 \mathrm{~d}^{4} \mathrm{D}$ \\
\hline 271296.05 & 270956 & 340 & 1.5 & $604 p^{4}\left({ }^{3} P\right) 4 d^{4} F+124 p^{4}\left({ }^{1} S\right) 4 d{ }^{2} D+104 p^{4}\left({ }^{3} P\right) 4 d{ }^{4} P$ \\
\hline 271374.36 & 270685 & 689 & 2.5 & $924 p^{4}\left({ }^{3} P\right) 4 d^{4} F$ \\
\hline 272091.26 & 272252 & -161 & 0.5 & $904 p^{4}\left({ }^{3} \mathrm{P}\right) 4 \mathrm{~d}^{4} \mathrm{P}$ \\
\hline 272834.44 & 273006 & -172 & 1.5 & $454 p^{4}\left({ }^{3} P\right) 4 d{ }^{4} P+234 p^{4}\left({ }^{3} P\right) 4 d{ }^{4} F+184 p^{4}\left({ }^{1} D\right) 4 d{ }^{2} P$ \\
\hline 274665.60 & 274850 & -184 & 1.5 & $384 \mathrm{p}^{4}\left({ }^{1} \mathrm{D}\right) 4 \mathrm{~d}^{2} \mathrm{D}+234 \mathrm{p}^{4}\left({ }^{3} \mathrm{P}\right) 4 \mathrm{~d}^{2} \mathrm{D}+104 \mathrm{p}^{4}\left({ }^{3} \mathrm{P}\right) 4 \mathrm{~d}^{2} \mathrm{P}$ \\
\hline 276491.34 & 276497 & -6 & 3.5 & $424 p^{4}\left({ }^{3} P\right) 4 d^{2} F+254 p^{4}\left({ }^{3} P\right) 4 d{ }^{4} F+204 p^{4}\left({ }^{1} D\right) 4 d^{2} G$ \\
\hline 278742.23 & 278849 & -107 & 2.5 & $734 p^{4}\left({ }^{3} P\right) 4 d^{4} P+94 p^{4}\left({ }^{1} S\right) 4 d^{2} D+74 p^{4}\left({ }^{3} P\right) 4 d^{2} F$ \\
\hline 279457.21 & 280229 & -772 & 1.5 & $394 p^{4}\left({ }^{3} P\right) 4 d^{4} P+244 p^{4}\left({ }^{1} D\right) 4 d^{2} P+224 p^{4}\left({ }^{3} P\right) 4 d^{2} P$ \\
\hline 283112.00 & 283096 & 16 & 2.5 & $384 p^{4}\left({ }^{1} S\right) 4 d^{2} D+204 p^{4}\left({ }^{3} P\right) 4 d^{2} D+194 p^{4}\left({ }^{3} P\right) 4 d{ }^{4} P$ \\
\hline 285967.09 & 285408 & 559 & 3.5 & $654 p^{4}\left({ }^{1} D\right) 4 d^{2} G+234 p^{4}\left({ }^{3} P\right) 4 d^{2} F+94 p^{4}\left({ }^{1} D\right) 4 d^{2} F$ \\
\hline 286411.50 & 285745 & 666 & 4.5 & $894 p^{4}\left({ }^{1} D\right) 4 d^{2} G+104 p^{4}\left({ }^{3} P\right) 4 d{ }^{4} F$ \\
\hline 287142.42 & 287582 & -440 & 2.5 & $614 p^{4}\left({ }^{3} P\right) 4 d^{2} F+204 p^{4}\left({ }^{1} D\right) 4 d^{2} F+114 p^{4}\left({ }^{1} D\right) 4 d^{2} D$ \\
\hline 299608.66 & 299907 & -298 & 2.5 & $764 p^{4}\left({ }^{1} D\right) 4 d^{2} F+124 p^{4}\left({ }^{3} P\right) 4 d^{2} F+94 p^{4}\left({ }^{1} D\right) 4 d^{2} D$ \\
\hline 303517.22 & 303778 & -260 & 3.5 & $804 p^{4}\left({ }^{1} D\right) 4 d^{2} F+164 p^{4}\left({ }^{3} P\right) 4 d^{2} F$ \\
\hline 319336.18 & 319348 & -11 & 1.5 & $624 p^{4}\left({ }^{1} S\right) 4 d^{2} D+254 p^{4}\left({ }^{1} D\right) 4 d^{2} D$ \\
\hline 325576.82 & 325455 & 121 & 2.5 & $724 p^{4}\left({ }^{1} S\right) 4 d^{2} D+134 p^{4}\left({ }^{1} D\right) 4 d^{2} D+54 p^{4}\left({ }^{3} P\right) 4 d^{2} F$ \\
\hline 334694.92 & 334643 & 52 & 0.5 & $704 p^{4}\left({ }^{1} D\right) 4 d^{2} S+184 s 4 p^{6}{ }^{2} S+54 p^{4}\left({ }^{1} D\right) 4 d^{2} P$ \\
\hline 339682.78 & 339148 & 535 & 1.5 & $494 p^{4}\left({ }^{3} P\right) 4 d^{2} P+364 p^{4}\left({ }^{1} D\right) 4 d^{2} P+74 p^{4}\left({ }^{1} D\right) 4 d^{2} D$ \\
\hline 343709.55 & 344545 & -835 & 2.5 & $644 p^{4}\left({ }^{3} P\right) 4 d^{2} D+224 p^{4}\left({ }^{1} D\right) 4 d^{2} D+104 p^{4}\left({ }^{1} S\right) 4 d^{2} D$ \\
\hline 346345.56 & 345413 & 932 & 0.5 & $474 p^{4}\left({ }^{3} P\right) 4 d^{2} P+414 p^{4}\left({ }^{1} D\right) 4 d^{2} P+84 p^{4}\left({ }^{1} D\right) 4 d^{2} S$ \\
\hline 358168.09 & 358487 & -319 & 1.5 & $564 p^{4}\left({ }^{3} P\right) 4 d^{2} D+184 p^{4}\left({ }^{1} S\right) 4 d^{2} D+154 p^{4}\left({ }^{1} D\right) 4 d^{2} D$ \\
\hline
\end{tabular}

Notes. Energies are given in $\mathrm{cm}^{-1} .{ }^{(a)}$ From Reader \& Lindsay (2016). ${ }^{(b)}$ This work. ${ }^{(c)}$ Only the first three components that are larger than 5\% are given. 
Table A.7. continued.

\begin{tabular}{|c|c|c|c|c|}
\hline$E_{\exp ^{a}}^{a}$ & $E_{\text {calc }}^{b}$ & $\Delta E$ & $J$ & Leading components (in \%) in $L S$ coupling $^{c}$ \\
\hline 364827.11 & 364808 & 19 & 2.5 & $914 p^{4}\left({ }^{3} P\right) 5 s{ }^{4} P+84 p^{4}\left({ }^{1} D\right) 5 s^{2} D$ \\
\hline 369711.65 & 369710 & 1 & 1.5 & $514 p^{4}\left({ }^{3} \mathrm{P}\right) 5 \mathrm{~s}{ }^{2} \mathrm{P}+384 \mathrm{p}^{4}\left({ }^{3} \mathrm{P}\right) 5 \mathrm{~s}{ }^{4} \mathrm{P}+104 \mathrm{p}^{4}\left({ }^{1} \mathrm{D}\right) 5 \mathrm{~s}{ }^{2} \mathrm{D}$ \\
\hline 377452.05 & 377510 & -58 & 0.5 & $904 p^{4}\left({ }^{3} P\right) 5 s{ }^{4} P+94 p^{4}\left({ }^{1} S\right) 5 s^{2} S$ \\
\hline 379776.65 & 379721 & 55 & 1.5 & $604 p^{4}\left({ }^{3} P\right) 5 s{ }^{4} P+364 p^{4}\left({ }^{3} P\right) 5 s{ }^{2} P$ \\
\hline 384781.44 & 384805 & -23 & 0.5 & $934 p^{4}\left({ }^{3} P\right) 5 s^{2} P+54 p^{4}\left({ }^{1} S\right) 5 s^{2} S$ \\
\hline 393555.34 & 393558 & -3 & 2.5 & $914 \mathrm{p}^{4}\left({ }^{1} \mathrm{D}\right) 5 \mathrm{~s}{ }^{2} \mathrm{D}+74 \mathrm{p}^{4}\left({ }^{3} \mathrm{P}\right) 5 \mathrm{~s}{ }^{4} \mathrm{P}$ \\
\hline 394195.47 & 394194 & 1 & 1.5 & $864 p^{4}\left({ }^{1} \mathrm{D}\right) 5 \mathrm{~s}^{2} \mathrm{D}+124 \mathrm{p}^{4}\left({ }^{3} \mathrm{P}\right) 5 \mathrm{~s}^{2} \mathrm{P}$ \\
\hline 423223.46 & 423216 & 7 & 0.5 & $834 p^{4}\left({ }^{1} S\right) 5 s^{2} S+84 p^{4}\left({ }^{3} P\right) 5 s{ }^{4} P+64 p^{4}\left({ }^{3} P\right) 5 s{ }^{2} P$ \\
\hline 514465.31 & 514326 & 140 & 2.5 & $714 p^{4}\left({ }^{3} P\right) 5 d{ }^{4} D+104 p^{4}\left({ }^{3} P\right) 5 d{ }^{4} F+94 p^{4}\left({ }^{3} P\right) 5 d{ }^{4} P$ \\
\hline 514487.01 & 514344 & 143 & 3.5 & $734 p^{4}\left({ }^{3} P\right) 5 d^{4} D+184 p^{4}\left({ }^{3} P\right) 5 d{ }^{4} F+64 p^{4}\left({ }^{1} D\right) 5 d^{2} F$ \\
\hline 515170.73 & 515071 & 100 & 1.5 & $604 p^{4}\left({ }^{3} P\right) 5 d^{4} D+194 p^{4}\left({ }^{3} P\right) 5 d^{4} P+64 p^{4}\left({ }^{1} D\right) 5 d^{2} D$ \\
\hline 516443.48 & 516466 & -22 & 0.5 & $454 p^{4}\left({ }^{3} P\right) 5 d^{4} D+254 p^{4}\left({ }^{3} P\right) 5 d{ }^{4} P+174 p^{4}\left({ }^{3} P\right) 5 d^{2} P$ \\
\hline 518061.55 & 517912 & 150 & 3.5 & $644 p^{4}\left({ }^{3} P\right) 5 d^{2} F+234 p^{4}\left({ }^{3} P\right) 5 d^{4} F+114 p^{4}\left({ }^{1} D\right) 5 d^{2} G$ \\
\hline 521740.06 & 521926 & -186 & 1.5 & $384 \mathrm{p}^{4}\left({ }^{3} \mathrm{P}\right) 5 \mathrm{~d}^{4} \mathrm{D}+344 \mathrm{p}^{4}\left({ }^{3} \mathrm{P}\right) 5 \mathrm{~d}^{2} \mathrm{D}+124 \mathrm{p}^{4}\left({ }^{3} \mathrm{P}\right) 5 \mathrm{~d}^{2} \mathrm{P}$ \\
\hline 522035.99 & 522139 & -103 & 2.5 & $394 \mathrm{p}^{4}\left({ }^{3} \mathrm{P}\right) 5 \mathrm{~d}^{2} \mathrm{D}+254 \mathrm{p}^{4}\left({ }^{3} \mathrm{P}\right) 5 \mathrm{~d}^{2} \mathrm{~F}+154 \mathrm{p}^{4}\left({ }^{3} \mathrm{P}\right) 5 \mathrm{~d}^{4} \mathrm{P}$ \\
\hline 528357.52 & 528376 & -19 & 0.5 & $504 \mathrm{p}^{4}\left({ }^{3} \mathrm{P}\right) 5 \mathrm{~d}^{4} \mathrm{D}+314 \mathrm{p}^{4}\left({ }^{3} \mathrm{P}\right) 5 \mathrm{~d}^{2} \mathrm{P}+94 \mathrm{p}^{4}\left({ }^{1} \mathrm{D}\right) 5 \mathrm{~d}^{2} \mathrm{P}$ \\
\hline 528976.13 & 528735 & 241 & 1.5 & $694 p^{4}\left({ }^{3} P\right) 5 d^{4} F+114 p^{4}\left({ }^{1} S\right) 5 d^{2} D+114 p^{4}\left({ }^{3} P\right) 5 d^{4} D$ \\
\hline 529351.71 & 529095 & 257 & 2.5 & $584 \mathrm{p}^{4}\left({ }^{3} \mathrm{P}\right) 5 \mathrm{~d}^{4} \mathrm{~F}+144 \mathrm{p}^{4}\left({ }^{3} \mathrm{P}\right) 5 \mathrm{~d}^{4} \mathrm{P}+124 \mathrm{p}^{4}\left({ }^{3} \mathrm{P}\right) 5 \mathrm{~d}^{4} \mathrm{D}$ \\
\hline 529945.22 & 529724 & 222 & 3.5 & $544 p^{4}\left({ }^{3} P\right) 5 d^{4} F+234 p^{4}\left({ }^{3} P\right) 5 d^{2} F+214 p^{4}\left({ }^{3} P\right) 5 d^{4} D$ \\
\hline 530538.91 & 530420 & 119 & 1.5 & $284 \mathrm{p}^{4}\left({ }^{3} \mathrm{P}\right) 5 \mathrm{~d}^{4} \mathrm{P}+254 \mathrm{p}^{4}\left({ }^{3} \mathrm{P}\right) 5 \mathrm{~d}^{4} \mathrm{D}+204 \mathrm{p}^{4}\left({ }^{3} \mathrm{P}\right) 5 \mathrm{~d}^{2} \mathrm{D}$ \\
\hline 532402.86 & 532261 & 142 & 2.5 & $524 \mathrm{p}^{4}\left({ }^{3} \mathrm{P}\right) 5 \mathrm{~d}^{4} \mathrm{P}+294 \mathrm{p}^{4}\left({ }^{3} \mathrm{P}\right) 5 \mathrm{~d}^{2} \mathrm{~F}+114 \mathrm{p}^{4}\left({ }^{3} \mathrm{P}\right) 5 \mathrm{~d}^{4} \mathrm{~F}$ \\
\hline 533736.95 & 533652 & 85 & 2.5 & $444 p^{4}\left({ }^{3} P\right) 5 d^{2} D+394 p^{4}\left({ }^{3} P\right) 5 d^{2} F$ \\
\hline 534552.78 & 534821 & -268 & 1.5 & $644 p^{4}\left({ }^{3} P\right) 5 d^{2} P+154 p^{4}\left({ }^{3} P\right) 5 d^{2} D+84 p^{4}\left({ }^{1} D\right) 5 d^{2} P$ \\
\hline 543295.84 & 543372 & -77 & 0.5 & $794 p^{4}\left({ }^{1} D\right) 5 d^{2} S+104 p^{4}\left({ }^{3} P\right) 5 d{ }^{4} P+94 p^{4}\left({ }^{1} D\right) 5 d^{2} P$ \\
\hline 544423.00 & 544411 & 12 & 1.5 & $734 p^{4}\left({ }^{1} \mathrm{D}\right) 5 \mathrm{~d}^{2} \mathrm{P}+84 \mathrm{p}^{4}\left({ }^{3} \mathrm{P}\right) 6 \mathrm{~s}^{2} \mathrm{P}+74 \mathrm{p}^{4}\left({ }^{3} \mathrm{P}\right) 5 \mathrm{~d}^{4} \mathrm{P}$ \\
\hline 545413.52 & 545407 & 7 & 2.5 & $904 \mathrm{p}^{4}\left({ }^{3} \mathrm{P}\right) 6 \mathrm{~s}{ }^{4} \mathrm{P}+84 \mathrm{p}^{4}\left({ }^{1} \mathrm{D}\right) 6 \mathrm{~s}^{2} \mathrm{D}$ \\
\hline 545666.07 & 545943 & -277 & 2.5 & $744 p^{4}\left({ }^{1} D\right) 5 d^{2} D+164 p^{4}\left({ }^{1} D\right) 5 d^{2} F$ \\
\hline 547213.94 & 547484 & -270 & 2.5 & $744 p^{4}\left({ }^{1} \mathrm{D}\right) 5 \mathrm{~d}^{2} \mathrm{~F}+144 \mathrm{p}^{4}\left({ }^{1} \mathrm{D}\right) 5 \mathrm{~d}^{2} \mathrm{D}+74 \mathrm{p}^{4}\left({ }^{3} \mathrm{P}\right) 5 \mathrm{~d}^{2} \mathrm{D}$ \\
\hline 547471.92 & 547470 & 2 & 1.5 & $634 p^{4}\left({ }^{3} \mathrm{P}\right) 6 \mathrm{~s}^{2} \mathrm{P}+204 \mathrm{p}^{4}\left({ }^{3} \mathrm{P}\right) 6 \mathrm{~s}{ }^{4} \mathrm{P}+84 \mathrm{p}^{4}\left({ }^{1} \mathrm{D}\right) 6 \mathrm{~s}^{2} \mathrm{D}$ \\
\hline 547791.00 & 548110 & -319 & 0.5 & $664 p^{4}\left({ }^{1} \mathrm{D}\right) 5 \mathrm{~d}^{2} \mathrm{P}+234 \mathrm{p}^{4}\left({ }^{3} \mathrm{P}\right) 5 \mathrm{~d}^{2} \mathrm{P}+74 \mathrm{p}^{4}\left({ }^{1} \mathrm{D}\right) 5 \mathrm{~d}^{2} \mathrm{~S}$ \\
\hline 548805.54 & 549467 & -661 & 1.5 & $784 p^{4}\left({ }^{1} D\right) 5 d^{2} D+184 p^{4}\left({ }^{3} P\right) 5 d^{2} D$ \\
\hline 558208.73 & 558215 & -6 & 0.5 & $864 p^{4}\left({ }^{3} \mathrm{P}\right) 6 \mathrm{~s}{ }^{4} \mathrm{P}+124 \mathrm{p}^{4}\left({ }^{1} \mathrm{~S}\right) 6 \mathrm{~s}^{2} \mathrm{~S}$ \\
\hline 559356.47 & 559344 & 13 & 1.5 & $784 \mathrm{p}^{4}\left({ }^{3} \mathrm{P}\right) 6 \mathrm{~s}{ }^{4} \mathrm{P}+224 \mathrm{p}^{4}\left({ }^{3} \mathrm{P}\right) 6 \mathrm{~s}^{2} \mathrm{P}$ \\
\hline 561050.32 & 561062 & -11 & 0.5 & $924 p^{4}\left({ }^{3} \mathrm{P}\right) 6 \mathrm{~s}^{2} \mathrm{P}$ \\
\hline 573101.84 & 572669 & 433 & 2.5 & $824 p^{4}\left({ }^{1} S\right) 5 d^{2} D$ \\
\hline 573301.14 & 573148 & 153 & 1.5 & $794 \mathrm{p}^{4}\left({ }^{1} \mathrm{~S}\right) 5 \mathrm{~d}^{2} \mathrm{D}+64 \mathrm{p}^{4}\left({ }^{3} \mathrm{P}\right) 5 \mathrm{~d}^{4} \mathrm{~F}$ \\
\hline 574494.88 & 574600 & -105 & 2.5 & $924 p^{4}\left({ }^{1} \mathrm{D}\right) 6 \mathrm{~s}^{2} \mathrm{D}+84 \mathrm{p}^{4}\left({ }^{3} \mathrm{P}\right) 6 \mathrm{~s}{ }^{4} \mathrm{P}$ \\
\hline 574889.14 & 574785 & 105 & 1.5 & $894 \mathrm{p}^{4}\left({ }^{1} \mathrm{D}\right) 6 \mathrm{~s}^{2} \mathrm{D}+84 \mathrm{p}^{4}\left({ }^{3} \mathrm{P}\right) 6 \mathrm{~s}^{2} \mathrm{P}$ \\
\hline 602661.00 & 602660 & 1 & 0.5 & $834 p^{4}\left({ }^{1} S\right) 6 s^{2} S+104 p^{4}\left({ }^{3} P\right) 6 s{ }^{4} P+54 p^{4}\left({ }^{3} P\right) 6 s^{2} P$ \\
\hline
\end{tabular}


Table A.8. Comparison between available experimental and calculated energy levels in Zr VII.

\begin{tabular}{|c|c|c|c|c|}
\hline$E_{\text {exp }}{ }^{a}$ & $E_{\text {calc }}^{b}$ & $\Delta E$ & $J$ & Leading components (in \%) in $L S$ coupling ${ }^{c}$ \\
\hline & & & & Even parity \\
\hline 0 & 2 & -2 & 2 & $894 p^{4}{ }^{3} \mathrm{P}+94 \mathrm{p}^{4}{ }^{1} \mathrm{D}$ \\
\hline 12557 & 12554 & 3 & 0 & $834 \mathrm{p}^{4}{ }^{3} \mathrm{P}+144 \mathrm{p}^{4}{ }^{1} \mathrm{~S}$ \\
\hline 13549 & 13550 & -1 & 1 & $974 \mathrm{p}^{4}{ }^{3} \mathrm{P}$ \\
\hline 27176 & 27176 & 0 & 2 & $884 \mathrm{p}^{4}{ }^{1} \mathrm{D}+94 \mathrm{p}^{4}{ }^{3} \mathrm{P}$ \\
\hline 56943 & 56943 & 0 & 0 & $844 p^{4}{ }^{1} \mathrm{~S}+144 \mathrm{p}^{4}{ }^{3} \mathrm{P}$ \\
\hline 480659 & 480829 & -170 & 1 & $534 p^{3}\left({ }^{4} S\right) 5 p^{3} P+114 p^{3}\left({ }^{4} S\right) 5 p{ }^{5} P+84 p^{3}\left({ }^{2} D\right) 5 p^{3} P$ \\
\hline 483891 & 484629 & -738 & 2 & $614 p^{3}\left({ }^{4} S\right) 5 p^{3} P+174 p^{3}\left({ }^{4} S\right) 5 p^{5} P+74 p^{3}\left({ }^{2} D\right) 5 p^{3} P$ \\
\hline 485937 & 484685 & 1252 & 0 & $844 p^{3}\left({ }^{4} S\right) 5 p^{3} P+74 p^{3}\left({ }^{2} P\right) 5 p^{3} P+64 p^{3}\left({ }^{2} D\right) 5 p^{3} P$ \\
\hline 492000 & 494297 & -2297 & 1 & $334 p^{3}\left({ }^{2} \mathrm{D}\right) 5 \mathrm{p}{ }^{1} \mathrm{P}+304 \mathrm{p}^{3}\left({ }^{2} \mathrm{D}\right) 5 \mathrm{p}^{3} \mathrm{D}+124 \mathrm{p}^{3}\left({ }^{4} \mathrm{~S}\right) 5 \mathrm{p}^{3} \mathrm{P}$ \\
\hline 498029 & 498816 & -787 & 2 & $484 p^{3}\left({ }^{2} \mathrm{D}\right) 5 \mathrm{p}^{3} \mathrm{~F}+274 \mathrm{p}^{3}\left({ }^{2} \mathrm{D}\right) 5 \mathrm{p}^{3} \mathrm{D}+114 \mathrm{p}^{3}\left({ }^{2} \mathrm{P}\right) 5 \mathrm{p}^{3} \mathrm{D}$ \\
\hline 501798 & 502258 & -460 & 3 & $644 p^{3}\left({ }^{2} D\right) 5 p^{3} F+144 p^{3}\left({ }^{2} D\right) 5 p^{3} D+124 p^{3}\left({ }^{2} P\right) 5 p^{3} D$ \\
\hline 504480 & 505161 & -681 & 3 & $744 p^{3}\left({ }^{2} \mathrm{D}\right) 5 \mathrm{p}^{1} \mathrm{~F}+174 \mathrm{p}^{3}\left({ }^{2} \mathrm{D}\right) 5 \mathrm{p}^{3} \mathrm{D}$ \\
\hline 504897 & 503622 & 1275 & 1 & $414 p^{3}\left({ }^{2} D\right) 5 p^{3} D+374 p^{3}\left({ }^{2} D\right) 5 p{ }^{1} P+124 p^{3}\left({ }^{2} P\right) 5 p^{3} D$ \\
\hline 506544 & 502353 & 4191 & 2 & $514 p^{3}\left({ }^{2} D\right) 5 p^{3} D+294 p^{3}\left({ }^{2} D\right) 5 p^{3} F+74 p^{3}\left({ }^{2} P\right) 5 p{ }^{1} D$ \\
\hline 507603 & 508524 & -921 & 4 & $754 p^{3}\left({ }^{2} D\right) 5 p{ }^{3} F+164 s 4 p^{4} 4 d^{1} G$ \\
\hline 507868 & 507908 & -40 & 3 & $644 p^{3}\left({ }^{2} \mathrm{D}\right) 5 \mathrm{p}^{3} \mathrm{D}+214 \mathrm{p}^{3}\left({ }^{2} \mathrm{D}\right) 5 \mathrm{p}^{3} \mathrm{~F}+104 \mathrm{p}^{3}\left({ }^{2} \mathrm{D}\right) 5 \mathrm{p}{ }^{1} \mathrm{~F}$ \\
\hline 512175 & 513167 & -992 & 2 & $574 p^{3}\left({ }^{2} \mathrm{D}\right) 5 \mathrm{p}^{3} \mathrm{P}+144 \mathrm{p}^{3}\left({ }^{2} \mathrm{P}\right) 5 \mathrm{p}^{3} \mathrm{P}+104 \mathrm{p}^{3}\left({ }^{4} \mathrm{~S}\right) 5 \mathrm{p}^{3} \mathrm{P}$ \\
\hline 515789 & 515584 & 205 & 0 & $804 p^{3}\left({ }^{2} D\right) 5 p{ }^{3} P+114 p^{3}\left({ }^{2} P\right) 5 p{ }^{1} S$ \\
\hline 522993 & 523937 & -944 & 1 & $374 p^{3}\left({ }^{2} P\right) 5 p^{3} D+94 p^{3}\left({ }^{2} P\right) 5 p{ }^{1} P+84 s 4 p^{4} 4 d^{3} D$ \\
\hline 524269 & 524312 & -43 & 2 & $534 p^{3}\left({ }^{2} \mathrm{D}\right) 5 p^{1} \mathrm{D}+104 \mathrm{p}^{3}\left({ }^{2} \mathrm{D}\right) 5 \mathrm{p}^{3} \mathrm{P}+64 \mathrm{~s} 4 \mathrm{p}^{4} 4 \mathrm{~d}^{3} \mathrm{D}$ \\
\hline 527639 & 528353 & -714 & 2 & $444 p^{3}\left({ }^{2} P\right) 5 p{ }^{3} D+104 p^{3}\left({ }^{4} S\right) 4 f^{3} F+74 s 4 p^{4} 4 d^{1} D$ \\
\hline 530030 & 530023 & 7 & 1 & $614 p^{3}\left({ }^{2} \mathrm{P}\right) 5 \mathrm{p}{ }^{3} \mathrm{P}+194 \mathrm{p}^{3}\left({ }^{2} \mathrm{P}\right) 5 \mathrm{p}{ }^{1} \mathrm{P}$ \\
\hline 530591 & 531023 & -432 & 0 & $854 p^{3}\left({ }^{2} P\right) 5 p^{3} P+64 p^{3}\left({ }^{4} S\right) 5 p^{3} P+54 p^{3}\left({ }^{2} P\right) 5 p{ }^{1} S$ \\
\hline 530672 & 530657 & 15 & 1 & $584 p^{3}\left({ }^{2} \mathrm{P}\right) 5 \mathrm{p}^{3} \mathrm{~S}+214 \mathrm{p}^{3}\left({ }^{2} \mathrm{D}\right) 5 \mathrm{p}^{3} \mathrm{P}+64 \mathrm{p}^{3}\left({ }^{2} \mathrm{D}\right) 5 \mathrm{p}{ }^{1} \mathrm{P}$ \\
\hline 534485 & 534639 & -154 & 3 & $684 p^{3}\left({ }^{2} \mathrm{P}\right) 5 \mathrm{p}^{3} \mathrm{D}+94 \mathrm{p}^{3}\left({ }^{2} \mathrm{D}\right) 5 \mathrm{p}^{3} \mathrm{~F}+84 \mathrm{p}^{3}\left({ }^{2} \mathrm{D}\right) 5 \mathrm{p}^{1} \mathrm{~F}$ \\
\hline 537188 & 537174 & 14 & 1 & $224 s 4 p^{4} 4 d^{3} D+194 p^{3}\left({ }^{4} S\right) 4 f^{5} F+104 s 4 p^{4} 4 d^{3} D$ \\
\hline 538927 & 537535 & 1392 & 2 & $634 p^{3}\left({ }^{2} \mathrm{P}\right) 5 \mathrm{p}^{1} \mathrm{D}+114 \mathrm{p}^{3}\left({ }^{2} \mathrm{D}\right) 5 \mathrm{p}{ }^{1} \mathrm{D}+84 \mathrm{p}^{3}\left({ }^{2} \mathrm{D}\right) 5 \mathrm{p}^{3} \mathrm{~F}$ \\
\hline 540660 & 542310 & -1650 & 2 & $334 p^{3}\left({ }^{2} P\right) 5 p^{3} P+124 s 4 p^{4} 4 d^{3} P+94 s 4 p^{4} 4 d^{3} P$ \\
\hline 542453 & 541036 & 1417 & 1 & $344 p^{3}\left({ }^{2} P\right) 5 p{ }^{1} P+204 p^{3}\left({ }^{2} P\right) 5 p^{3} P+104 p^{3}\left({ }^{2} D\right) 5 p^{3} D$ \\
\hline 556807 & 556867 & -60 & 0 & $\begin{array}{l}634 p^{3}\left({ }^{2} P\right) 5 p{ }^{1} S+74 p^{3}\left({ }^{2} D\right) 5 p{ }^{3} P+54 s 4 p^{4} 4 d^{3} P \\
\text { Odd parity }\end{array}$ \\
\hline 192812 & 192785 & 27 & 2 & $864 s 4 p^{5}{ }^{3} P+94 p^{3}\left({ }^{2} D\right) 4 d^{3} P$ \\
\hline 201981 & 202001 & -21 & 1 & $834 s 4 p^{5}{ }^{3} P+94 p^{3}\left({ }^{2} D\right) 4 d^{3} P$ \\
\hline 208638 & 208552 & 86 & 0 & $854 \mathrm{~s} 4 \mathrm{p}^{5}{ }^{3} \mathrm{P}+104 \mathrm{p}^{3}\left({ }^{2} \mathrm{D}\right) 4 \mathrm{~d}^{3} \mathrm{P}$ \\
\hline 243704 & 243873 & -169 & 1 & $644 s 4 p^{5}{ }^{1} \mathrm{P}+274 \mathrm{p}^{3}\left({ }^{2} \mathrm{D}\right) 4 \mathrm{~d}^{1} \mathrm{P}$ \\
\hline 262683 & 263032 & -349 & 0 & $954 p^{3}\left({ }^{4} S\right) 4 d^{5} D$ \\
\hline 263119 & 263287 & -168 & 1 & $964 p^{3}\left({ }^{4} S\right) 4 d^{5} D$ \\
\hline 263702 & 263263 & 439 & 2 & $924 p^{3}\left({ }^{4} S\right) 4 d^{5} D$ \\
\hline 264081 & 263321 & 760 & 3 & $894 p^{3}\left({ }^{4} S\right) 4 d^{5} D$ \\
\hline 264903 & 264332 & 571 & 4 & $934 p^{3}\left({ }^{4} S\right) 4 d^{5} \mathrm{D}+54 \mathrm{p}^{3}\left({ }^{2} \mathrm{P}\right) 4 \mathrm{~d}^{3} \mathrm{~F}$ \\
\hline 275418 & 276399 & -981 & 2 & $244 p^{3}\left({ }^{4} S\right) 4 d^{3} D+234 p^{3}\left({ }^{2} D\right) 4 d^{3} D+234 p^{3}\left({ }^{2} D\right) 4 d^{3} F$ \\
\hline 280850 & 281217 & -367 & 3 & $344 p^{3}\left({ }^{2} D\right) 4 d^{3} D+314 p^{3}\left({ }^{4} S\right) 4 d^{3} D+124 p^{3}\left({ }^{2} D\right) 4 d^{3} F$ \\
\hline 282419 & 283129 & -710 & 1 & $474 p^{3}\left({ }^{2} D\right) 4 d^{3} D+454 p^{3}\left({ }^{4} S\right) 4 d^{3} D$ \\
\hline 285543 & 285392 & 151 & 2 & $484 p^{3}\left({ }^{2} D\right) 4 d^{3} F+234 p^{3}\left({ }^{2} D\right) 4 d^{3} D+154 p^{3}\left({ }^{4} S\right) 4 d^{3} D$ \\
\hline 288053 & 287594 & 459 & 3 & $574 \mathrm{p}^{3}\left({ }^{2} \mathrm{D}\right) 4 \mathrm{~d}^{3} \mathrm{~F}+144 \mathrm{p}^{3}\left({ }^{2} \mathrm{D}\right) 4 \mathrm{~d}^{3} \mathrm{D}+124 \mathrm{p}^{3}\left({ }^{2} \mathrm{P}\right) 4 \mathrm{~d}^{3} \mathrm{~F}$ \\
\hline 289300 & 290371 & -1071 & 0 & $934 p^{3}\left({ }^{2} D\right) 4 d^{1} S$ \\
\hline 291472 & 290767 & 705 & 4 & $624 p^{3}\left({ }^{2} D\right) 4 d^{3} F+164 p^{3}\left({ }^{2} D\right) 4 d^{3} G+154 p^{3}\left({ }^{2} P\right) 4 d^{3} F$ \\
\hline 296679 & 296182 & 497 & 3 & $814 p^{3}\left({ }^{2} D\right) 4 d^{3} G+124 p^{3}\left({ }^{2} D\right) 4 d^{3} F$ \\
\hline 298282 & 298336 & -54 & 4 & $674 p^{3}\left({ }^{2} \mathrm{D}\right) 4 d^{3} \mathrm{G}+284 \mathrm{p}^{3}\left({ }^{2} \mathrm{D}\right) 4 \mathrm{~d}^{3} \mathrm{~F}$ \\
\hline 300720 & 300635 & 85 & 5 & $994 p^{3}\left({ }^{2} \mathrm{D}\right) 4 \mathrm{~d}^{3} \mathrm{G}$ \\
\hline
\end{tabular}

Notes. Energies are given in $\mathrm{cm}^{-1}$. ${ }^{(a)}$ From Reader \& Acquista (1976), Rahimullah et al. (1978), and Khan et al. (1983). ${ }^{(b)}$ This work. ${ }^{(c)}$ Only the first three components that are larger than $5 \%$ are given. 
T. Rauch et al.: Stellar laboratories. VIII.

Table A.8. continued.

\begin{tabular}{|c|c|c|c|c|}
\hline$E_{\text {exp }}^{a}$ & $E_{\text {calc }}^{b}$ & $\Delta E$ & $J$ & Leading components (in \%) in $L S$ coupling ${ }^{c}$ \\
\hline 303437 & 303512 & -75 & 4 & $844 p^{3}\left({ }^{2} D\right) 4 d^{1} G+74 p^{3}\left({ }^{2} D\right) 4 d^{3} G$ \\
\hline 311985 & 311104 & 881 & 2 & $534 p^{3}\left({ }^{2} \mathrm{P}\right) 4 d^{1} \mathrm{D}+214 \mathrm{p}^{3}\left({ }^{2} \mathrm{D}\right) 4 \mathrm{~d}^{1} \mathrm{D}+114 \mathrm{p}^{3}\left({ }^{2} \mathrm{P}\right) 4 \mathrm{~d}^{3} \mathrm{~F}$ \\
\hline 312987 & 313638 & -651 & 1 & $514 p^{3}\left({ }^{2} P\right) 4 d^{3} D+324 p^{3}\left({ }^{2} D\right) 4 d^{3} D+124 p^{3}\left({ }^{4} S\right) 4 d^{3} D$ \\
\hline 317400 & 319578 & -2178 & 0 & $634 \mathrm{p}^{3}\left({ }^{2} \mathrm{P}\right) 4 \mathrm{~d}^{3} \mathrm{P}+274 \mathrm{p}^{3}\left({ }^{2} \mathrm{D}\right) 4 \mathrm{~d}^{3} \mathrm{P}+64 \mathrm{p}^{3}\left({ }^{2} \mathrm{D}\right) 4 \mathrm{~d}^{1} \mathrm{~S}$ \\
\hline 320989 & 321259 & -270 & 2 & $494 p^{3}\left({ }^{2} P\right) 4 d^{3} D+194 p^{3}\left({ }^{2} D\right) 4 d^{3} D+124 p^{3}\left({ }^{4} S\right) 4 d^{3} D$ \\
\hline 322407 & 322588 & -181 & 3 & $734 p^{3}\left({ }^{2} P\right) 4 d^{3} F+124 p^{3}\left({ }^{2} D\right) 4 d^{3} F+84 p^{3}\left({ }^{2} D\right) 4 d^{3} G$ \\
\hline 323711 & 324292 & -581 & 2 & $554 \mathrm{p}^{3}\left({ }^{2} \mathrm{P}\right) 4 \mathrm{~d}^{3} \mathrm{~F}+234 \mathrm{p}^{3}\left({ }^{2} \mathrm{D}\right) 4 \mathrm{~d}^{3} \mathrm{~F}+64 \mathrm{p}^{3}\left({ }^{2} \mathrm{D}\right) 4 \mathrm{~d}^{3} \mathrm{D}$ \\
\hline 323870 & 320328 & 3542 & 1 & $684 p^{3}\left({ }^{2} P\right) 4 d^{3} P+174 p^{3}\left({ }^{2} D\right) 4 d^{3} P+64 p^{3}\left({ }^{2} D\right) 4 d^{3} S$ \\
\hline 324907 & 325653 & -746 & 4 & $704 p^{3}\left({ }^{2} P\right) 4 d^{3} F+124 p^{3}\left({ }^{2} D\right) 4 d^{1} G+84 p^{3}\left({ }^{2} D\right) 4 d^{3} G$ \\
\hline 328276 & 328706 & -430 & 3 & $374 \mathrm{p}^{3}\left({ }^{2} \mathrm{P}\right) 4 \mathrm{~d}^{3} \mathrm{D}+314 \mathrm{p}^{3}\left({ }^{2} \mathrm{D}\right) 4 \mathrm{~d}^{3} \mathrm{D}+114 \mathrm{p}^{3}\left({ }^{2} \mathrm{P}\right) 4 \mathrm{~d}^{1} \mathrm{~F}$ \\
\hline 330126 & 330701 & -575 & 2 & $794 \mathrm{p}^{3}\left({ }^{2} \mathrm{P}\right) 4 \mathrm{~d}^{3} \mathrm{P}+54 \mathrm{p}^{3}\left({ }^{2} \mathrm{P}\right) 4 \mathrm{~d}^{1} \mathrm{D}$ \\
\hline 342695 & 340697 & 1998 & 1 & $834 \mathrm{p}^{3}\left({ }^{2} \mathrm{D}\right) 4 \mathrm{~d}^{3} \mathrm{~S}+134 \mathrm{p}^{3}\left({ }^{2} \mathrm{D}\right) 4 \mathrm{~d}^{3} \mathrm{P}$ \\
\hline 343828 & 344828 & -1000 & 2 & $824 p^{3}\left({ }^{2} \mathrm{D}\right) 4 \mathrm{~d}^{3} \mathrm{P}+104 \mathrm{~s} 4 \mathrm{p}^{5}{ }^{3} \mathrm{P}$ \\
\hline 345215 & 344686 & 529 & 1 & $424 \mathrm{p}^{3}\left({ }^{2} \mathrm{D}\right) 4 \mathrm{~d}^{1} \mathrm{P}+234 \mathrm{p}^{3}\left({ }^{2} \mathrm{D}\right) 4 \mathrm{~d}^{3} \mathrm{P}+204 \mathrm{~s} 4 \mathrm{p}^{5}{ }^{1} \mathrm{P}$ \\
\hline 346462 & 345598 & 864 & 3 & $514 p^{3}\left({ }^{2} P\right) 4 d^{1} F+204 p^{3}\left({ }^{2} D\right) 4 d^{1} F+194 p^{3}\left({ }^{2} P\right) 4 d^{3} D$ \\
\hline 352853 & 353419 & -566 & 3 & $424 p^{3}\left({ }^{4} S\right) 4 d^{3} D+264 p^{3}\left({ }^{2} P\right) 4 d^{3} D+164 p^{3}\left({ }^{2} D\right) 4 d^{3} D$ \\
\hline 354335 & 354703 & -368 & 1 & $354 \mathrm{p}^{3}\left({ }^{2} \mathrm{D}\right) 4 \mathrm{~d}^{3} \mathrm{P}+234 \mathrm{p}^{3}\left({ }^{2} \mathrm{D}\right) 4 \mathrm{~d}^{1} \mathrm{P}+194 \mathrm{p}^{3}\left({ }^{2} \mathrm{P}\right) 4 \mathrm{~d}^{3} \mathrm{P}$ \\
\hline 355650 & 355413 & 237 & 0 & $614 p^{3}\left({ }^{2} \mathrm{D}\right) 4 \mathrm{~d}^{3} \mathrm{P}+244 \mathrm{p}^{3}\left({ }^{2} \mathrm{P}\right) 4 \mathrm{~d}^{3} \mathrm{P}+144 \mathrm{~s} 4 \mathrm{p}^{5}{ }^{3} \mathrm{P}$ \\
\hline 360177 & 360333 & -156 & 2 & $304 p^{3}\left({ }^{4} S\right) 4 d^{3} D+274 p^{3}\left({ }^{2} P\right) 4 d^{3} D+164 p^{3}\left({ }^{2} D\right) 4 d^{1} D$ \\
\hline 364897 & 364861 & 36 & 1 & $394 p^{3}\left({ }^{2} P\right) 4 d^{3} D+354 p^{3}\left({ }^{4} S\right) 4 d^{3} D+154 p^{3}\left({ }^{2} D\right) 4 d^{3} D$ \\
\hline 371371 & 371578 & -207 & 2 & $544 \mathrm{p}^{3}\left({ }^{2} \mathrm{D}\right) 4 \mathrm{~d}^{1} \mathrm{D}+194 \mathrm{p}^{3}\left({ }^{2} \mathrm{P}\right) 4 \mathrm{~d}^{1} \mathrm{D}+114 \mathrm{p}^{3}\left({ }^{2} \mathrm{P}\right) 4 \mathrm{~d}^{3} \mathrm{D}$ \\
\hline 380360 & 380849 & -489 & 3 & $594 \mathrm{p}^{3}\left({ }^{2} \mathrm{D}\right) 4 \mathrm{~d}^{1} \mathrm{~F}+314 \mathrm{p}^{3}\left({ }^{2} \mathrm{P}\right) 4 \mathrm{~d}^{1} \mathrm{~F}$ \\
\hline 397987 & 397488 & 499 & 1 & $824 \mathrm{p}^{3}\left({ }^{2} \mathrm{P}\right) 4 \mathrm{~d}^{1} \mathrm{P}$ \\
\hline 408775 & 408782 & -7 & 2 & $914 \mathrm{p}^{3}\left({ }^{4} \mathrm{~S}\right) 5 \mathrm{~s}{ }^{5} \mathrm{~S}+74 \mathrm{p}^{3}\left({ }^{2} \mathrm{P}\right) 5 \mathrm{~s}{ }^{3} \mathrm{P}$ \\
\hline 418375 & 418373 & 2 & 1 & $854 \mathrm{p}^{3}\left({ }^{4} \mathrm{~S}\right) 5 \mathrm{~s}{ }^{3} \mathrm{~S}+64 \mathrm{p}^{3}\left({ }^{2} \mathrm{P}\right) 5 \mathrm{~s}{ }^{1} \mathrm{P}$ \\
\hline 434766 & 434714 & 52 & 2 & $684 p^{3}\left({ }^{2} \mathrm{D}\right) 5 \mathrm{~s}^{3} \mathrm{D}+154 \mathrm{p}^{3}\left({ }^{2} \mathrm{P}\right) 5 \mathrm{~s}{ }^{3} \mathrm{P}+104 \mathrm{p}^{3}\left({ }^{2} \mathrm{D}\right) 5 \mathrm{~s}{ }^{1} \mathrm{D}$ \\
\hline 434815 & 434803 & 12 & 1 & $794 p^{3}\left({ }^{2} \mathrm{D}\right) 5 \mathrm{~s}^{3} \mathrm{D}+94 \mathrm{p}^{3}\left({ }^{4} \mathrm{~S}\right) 5 \mathrm{~s}{ }^{3} \mathrm{~S}+64 \mathrm{p}^{3}\left({ }^{2} \mathrm{P}\right) 5 \mathrm{~s}{ }^{1} \mathrm{P}$ \\
\hline 439534 & 439566 & -32 & 3 & $994 p^{3}\left({ }^{2} D\right) 5 s{ }^{3} D$ \\
\hline 443204 & 443228 & -24 & 2 & $774 p^{3}\left({ }^{2} \mathrm{D}\right) 5 \mathrm{~s}^{1} \mathrm{D}+194 \mathrm{p}^{3}\left({ }^{2} \mathrm{D}\right) 5 \mathrm{~s}{ }^{3} \mathrm{D}$ \\
\hline 456721 & 456722 & -1 & 0 & $984 \mathrm{p}^{3}\left({ }^{2} \mathrm{P}\right) 5 \mathrm{~s}{ }^{3} \mathrm{P}$ \\
\hline 458043 & 458073 & -30 & 1 & $784 \mathrm{p}^{3}\left({ }^{2} \mathrm{P}\right) 5 \mathrm{~s}{ }^{3} \mathrm{P}+184 \mathrm{p}^{3}\left({ }^{2} \mathrm{P}\right) 5 \mathrm{~s}{ }^{1} \mathrm{P}$ \\
\hline 466123 & 466108 & 15 & 2 & $734 \mathrm{p}^{3}\left({ }^{2} \mathrm{P}\right) 5 \mathrm{~s}{ }^{3} \mathrm{P}+114 \mathrm{p}^{3}\left({ }^{2} \mathrm{D}\right) 5 \mathrm{~s}{ }^{1} \mathrm{D}+114 \mathrm{p}^{3}\left({ }^{2} \mathrm{D}\right) 5 \mathrm{~s}{ }^{3} \mathrm{D}$ \\
\hline 469225 & 469212 & 13 & 1 & $674 p^{3}\left({ }^{2} P\right) 5 s{ }^{1} P+144 p^{3}\left({ }^{2} D\right) 5 s^{3} D+114 p^{3}\left({ }^{2} P\right) 5 s^{3} P$ \\
\hline
\end{tabular}




\section{Appendix B: Additional tables for xenon}

Table B.1. Radial parameters (in $\mathrm{cm}^{-1}$ ) adopted for the calculations in Xe IV.

\begin{tabular}{|c|c|c|c|c|c|}
\hline Configuration & Parameter & HFR & Fitted & Ratio & Note $^{a}$ \\
\hline \multicolumn{6}{|c|}{ Odd parity } \\
\hline \multirow[t]{4}{*}{$5 p^{3}$} & $E_{\mathrm{av}}$ & 29571 & 29733 & & \\
\hline & $\mathrm{F}^{2}(5 \mathrm{p}, 5 \mathrm{p})$ & 53594 & 48082 & 0.897 & \\
\hline & $\alpha$ & 0 & -105 & & \\
\hline & $\zeta_{5 p}$ & 8331 & 9017 & 1.082 & \\
\hline \multirow[t]{8}{*}{$5 p^{2} 6 p$} & $E_{\mathrm{av}}$ & 220434 & 210991 & & \\
\hline & $F^{2}(5 p, 5 p)$ & 55341 & 42311 & 0.764 & \\
\hline & $\alpha$ & 0 & -136 & & \\
\hline & $\zeta_{5 p}$ & 9030 & 9306 & 1.031 & \\
\hline & $\zeta_{6 p}$ & 1957 & 2359 & 1.205 & \\
\hline & $\mathrm{F}^{2}(5 \mathrm{p}, 6 \mathrm{p})$ & 16960 & 13633 & 0.804 & \\
\hline & $\mathrm{G}^{0}(5 \mathrm{p}, 6 \mathrm{p})$ & 3574 & 2628 & 0.735 & \\
\hline & $G^{2}(5 p, 6 p)$ & 4748 & 2881 & 0.608 & \\
\hline \multirow[t]{9}{*}{$5 p^{2} 4 f$} & $E_{\mathrm{av}}$ & 219539 & 210573 & & \\
\hline & $\mathrm{F}^{2}(5 \mathrm{p}, 5 \mathrm{p})$ & 53413 & 38692 & 0.724 & \\
\hline & $\alpha$ & 0 & 570 & & \\
\hline & $\zeta_{4 f}$ & 126 & 126 & 1.000 & $\mathrm{~F}$ \\
\hline & $\zeta_{5 p}$ & 8239 & 8513 & 1.033 & \\
\hline & $\mathrm{F}^{2}(5 \mathrm{p}, 4 \mathrm{f})$ & 44254 & 38007 & 0.859 & \\
\hline & $G^{2}(5 p, 4 f)$ & 35873 & 31088 & 0.867 & \\
\hline & $G^{4}(5 p, 4 f)$ & 25004 & 18475 & 0.739 & \\
\hline & & n parity & & & \\
\hline \multirow[t]{5}{*}{$5 s 5 p^{4}$} & $E_{\mathrm{av}}$ & 145882 & 139362 & & \\
\hline & $\mathrm{F}^{2}(5 \mathrm{p}, 5 \mathrm{p})$ & 53665 & 48881 & 0.911 & \\
\hline & $\alpha$ & 0 & -398 & & \\
\hline & $\zeta_{5 p}$ & 8332 & 9009 & 1.081 & \\
\hline & $\mathrm{G}^{1}(5 \mathrm{~s}, 5 \mathrm{p})$ & 70466 & 50020 & 0.710 & \\
\hline \multirow[t]{8}{*}{$5 p^{2} 5 d$} & $E_{\mathrm{av}}$ & 171129 & 166438 & & \\
\hline & $\mathrm{F}^{2}(5 \mathrm{p}, 5 \mathrm{p})$ & 54305 & 36560 & 0.673 & \\
\hline & $\alpha$ & 0 & 484 & & \\
\hline & $\zeta_{5 p}$ & 8633 & 9163 & 1.061 & \\
\hline & $\zeta_{5 d}$ & 488 & 488 & 1.000 & $\mathrm{~F}$ \\
\hline & $\mathrm{F}^{2}(5 \mathrm{p}, 5 \mathrm{~d})$ & 40094 & 33282 & 0.830 & \\
\hline & $\mathrm{G}^{1}(5 \mathrm{p}, 5 \mathrm{~d})$ & 45506 & 35398 & 0.778 & \\
\hline & $\mathrm{G}^{3}(5 \mathrm{p}, 5 \mathrm{~d})$ & 28625 & 21026 & 0.734 & \\
\hline \multirow[t]{5}{*}{$5 p^{2} 6 s$} & $E_{\mathrm{av}}$ & 188047 & 178843 & & \\
\hline & $\mathrm{F}^{2}(5 \mathrm{p}, 5 \mathrm{p})$ & 54876 & 42392 & 0.772 & \\
\hline & $\alpha$ & 0 & -251 & & \\
\hline & $\zeta_{5 p}$ & 8890 & 9384 & 1.056 & \\
\hline & $\mathrm{G}^{1}(5 \mathrm{p}, 5 \mathrm{~d})$ & 6038 & 3450 & 0.571 & \\
\hline $5 s 5 p^{4}-5 p^{2} 5 d$ & $\mathrm{R}^{1}(5 \mathrm{p} 5 \mathrm{p} ; 5 \mathrm{~s} 5 \mathrm{~d})$ & 54354 & 42310 & 0.778 & \\
\hline $5 s 5 p^{4}-5 p^{2} 6 s$ & $\mathrm{R}^{1}(5 \mathrm{p} 5 \mathrm{p} ; 5 \mathrm{~s} 6 \mathrm{~s})$ & -1248 & -1123 & 0.900 & $\mathrm{~F}$ \\
\hline \multirow[t]{2}{*}{$5 p^{2} 5 d-5 p^{2} 6 s$} & $\mathrm{R}^{2}(5 \mathrm{p} 5 \mathrm{~d} ; 5 \mathrm{p} 6 \mathrm{~s})$ & -12911 & -8781 & 0.680 & $\mathrm{R}$ \\
\hline & $\mathrm{R}^{1}(5 \mathrm{p} 5 \mathrm{~d} ; 5 \mathrm{p} 6 \mathrm{~s})$ & -5224 & -3553 & 0.680 & $\mathrm{R}$ \\
\hline
\end{tabular}

Notes. ${ }^{(a)}$ F: Fixed parameter value; R: ratios of these parameters had been fixed in the fitting process. 
T. Rauch et al.: Stellar laboratories. VIII.

Table B.2. Radial parameters (in $\mathrm{cm}^{-1}$ ) adopted for the calculations in $\mathrm{Xe} \mathrm{V}$.

\begin{tabular}{|c|c|c|c|c|c|}
\hline Configuration & Parameter & HFR & Fitted & Ratio & Note $^{a}$ \\
\hline & & $\mathrm{n}$ parity & & & \\
\hline \multirow{4}{*}{$5 p^{2}$} & $E_{\mathrm{av}}$ & 28481 & 29415 & & \\
\hline & $F^{2}(5 p, 5 p)$ & 55631 & 50605 & 0.910 & \\
\hline & $\alpha$ & 0 & -111 & & \\
\hline & $\zeta_{5 p}$ & 9121 & 9730 & 1.067 & \\
\hline \multirow[t]{6}{*}{$5 p 6 p$} & $E_{\mathrm{av}}$ & 253537 & 252141 & & \\
\hline & $\zeta_{5 p}$ & 9817 & 8816 & 0.898 & \\
\hline & $\zeta_{6 p}$ & 2614 & 2694 & 1.031 & \\
\hline & $\mathrm{F}^{2}(5 \mathrm{p}, 6 \mathrm{p})$ & 19855 & 17257 & 0.869 & \\
\hline & $\mathrm{G}^{0}(5 \mathrm{p}, 6 \mathrm{p})$ & 4298 & 3568 & 0.830 & \\
\hline & $G^{2}(5 p, 6 p)$ & 5684 & 5510 & 0.969 & \\
\hline \multirow[t]{7}{*}{$5 \mathrm{p} 4 \mathrm{f}$} & $E_{\mathrm{av}}$ & 216146 & 210715 & & \\
\hline & $\zeta_{4 f}$ & 178 & 178 & 1.000 & $\mathrm{~F}$ \\
\hline & $\zeta_{5 p}$ & 8855 & 9606 & 1.085 & \\
\hline & $F^{2}(5 p, 4 f)$ & 48109 & 40305 & 0.838 & \\
\hline & $\mathrm{G}^{2}(5 \mathrm{p}, 4 \mathrm{f})$ & 36992 & 31844 & 0.861 & \\
\hline & $G^{4}(5 p, 4 f)$ & 26416 & 20142 & 0.762 & \\
\hline & & parity & & & \\
\hline \multirow[t]{5}{*}{$5 s 5 p^{3}$} & $E_{\mathrm{av}}$ & 142485 & 142543 & & \\
\hline & $F^{2}(5 p, 5 p)$ & 55684 & 47870 & 0.860 & \\
\hline & $\alpha$ & 0 & 100 & & \\
\hline & $\zeta_{5 p}$ & 9110 & 9952 & 1.092 & \\
\hline & $\mathrm{G}^{1}(5 \mathrm{~s}, 5 \mathrm{p})$ & 72784 & 55634 & 0.764 & \\
\hline \multirow{6}{*}{$5 \mathrm{p} 5 \mathrm{~d}$} & $E_{\mathrm{av}}$ & 184432 & 183499 & & \\
\hline & $\zeta_{5 p}$ & 9384 & 10095 & 1.076 & \\
\hline & $\zeta_{5 d}$ & 608 & 863 & 1.418 & \\
\hline & $\mathrm{F}^{2}(5 \mathrm{p}, 5 \mathrm{~d})$ & 43728 & 37678 & 0.862 & \\
\hline & $\mathrm{G}^{1}(5 \mathrm{p}, 5 \mathrm{~d})$ & 50314 & 39579 & 0.787 & \\
\hline & $G^{3}(5 p, 5 d)$ & 31869 & 25524 & 0.801 & \\
\hline \multirow[t]{6}{*}{$5 \mathrm{p} 6 \mathrm{~d}$} & $E_{\mathrm{av}}$ & 307421 & 305556 & & \\
\hline & $\zeta_{5 p}$ & 9805 & 9331 & 0.952 & \\
\hline & $\zeta_{6 d}$ & 233 & 233 & 1.000 & $\mathrm{~F}$ \\
\hline & $\mathrm{F}^{2}(5 \mathrm{p}, 6 \mathrm{~d})$ & 14535 & 11608 & 0.799 & \\
\hline & $\mathrm{G}^{1}(5 \mathrm{p}, 6 \mathrm{~d})$ & 8677 & 10765 & 1.241 & \\
\hline & $G^{3}(5 p, 6 d)$ & 6311 & 7518 & 1.191 & \\
\hline \multirow[t]{3}{*}{$5 \mathrm{p} 6 \mathrm{~s}$} & $E_{\mathrm{av}}$ & 215033 & 214915 & & \\
\hline & $\zeta_{5 p}$ & 9664 & 10297 & 1.066 & \\
\hline & $\mathrm{G}^{\mathrm{i}}(5 \mathrm{p}, 6 \mathrm{~s})$ & 6714 & 6378 & 0.950 & \\
\hline \multirow[t]{3}{*}{$5 \mathrm{p} 7 \mathrm{~s}$} & $E_{\mathrm{av}}$ & 317520 & 308416 & & \\
\hline & $\zeta_{5 p}$ & 9833 & 10172 & 1.034 & \\
\hline & $\mathrm{G}^{\mathrm{i}}(5 \mathrm{p}, 7 \mathrm{~s})$ & 2108 & 2015 & 0.956 & \\
\hline $5 s 5 p^{3}-5 p 5 d$ & $R^{1}(5 \mathrm{p} 5 \mathrm{p} ; 5 \mathrm{~s} 5 \mathrm{~d})$ & 58429 & 46328 & 0.793 & \\
\hline $5 s 5 p^{3}-5 p 6 s$ & $\mathrm{R}^{1}(5 \mathrm{p} 5 \mathrm{p} ; 5 \mathrm{~s} 6 \mathrm{~s})$ & -1254 & -1129 & 0.900 & $\mathrm{~F}$ \\
\hline \multirow[t]{2}{*}{$5 p 5 d-5 p 6 s$} & $\mathrm{R}^{2}(5 \mathrm{p} 5 \mathrm{~d} ; 5 \mathrm{p} 6 \mathrm{~s})$ & -13325 & -12401 & 0.931 & $\mathrm{R}$ \\
\hline & $\mathrm{R}^{1}(5 \mathrm{p} 5 \mathrm{~d} ; 5 \mathrm{p} 6 \mathrm{~s})$ & -5408 & -5033 & 0.931 & $\mathrm{R}$ \\
\hline
\end{tabular}

Notes. ${ }^{(a)}$ F: Fixed parameter value; R: ratios of these parameters have been fixed in the fitting process. 
Table B.3. Comparison between available experimental and calculated energy levels in Xe IV.

\begin{tabular}{|c|c|c|c|c|}
\hline$E_{\text {exp }}^{a}$ & $E_{\text {calc }}^{b}$ & $\Delta E$ & $J$ & Leading components (in \%) in $L S$ coupling ${ }^{c}$ \\
\hline & & & & Odd parity \\
\hline 0.0 & 3 & -3 & 1.5 & $815 p^{3}{ }^{4} \mathrm{~S}+125 \mathrm{p}^{32} \mathrm{P}$ \\
\hline 13267.0 & 13248 & 19 & 1.5 & $725 \mathrm{p}^{32} \mathrm{D}+135 \mathrm{p}^{32} \mathrm{P}+115 \mathrm{p}^{3}{ }^{4} \mathrm{~S}$ \\
\hline 17510.7 & 17524 & -13 & 2.5 & $965 \mathrm{p}^{32} \mathrm{D}$ \\
\hline 28036.4 & 28043 & -7 & 0.5 & $965 \mathrm{p}^{32} \mathrm{P}$ \\
\hline 35649.6 & 35644 & 5 & 1.5 & $705 \mathrm{p}^{32} \mathrm{P}+205 \mathrm{p}^{32} \mathrm{D}+55 \mathrm{p}^{3{ }^{4}} \mathrm{~s}$ \\
\hline 180151.5 & 180062 & 90 & 2.5 & $685 \mathrm{p}^{2}\left({ }^{3} \mathrm{P}\right) 4 \mathrm{f}^{4} \mathrm{G}+75 \mathrm{p}^{2}\left({ }^{1} \mathrm{~S}\right) 4 \mathrm{f}^{2} \mathrm{~F}+75 \mathrm{p}^{2}\left({ }^{3} \mathrm{P}\right) 4 \mathrm{f}^{4} \mathrm{~F}$ \\
\hline 182219.1 & 182422 & -203 & 3.5 & $505 p^{2}\left({ }^{3} \mathrm{P}\right) 4 \mathrm{f}^{4} \mathrm{G}+145 \mathrm{p}^{2}\left({ }^{3} \mathrm{P}\right) 4 \mathrm{f}^{4} \mathrm{D}+145 \mathrm{p}^{2}\left({ }^{3} \mathrm{P}\right) 4 \mathrm{f}^{4} \mathrm{~F}$ \\
\hline 186109.1 & 186093 & 16 & 0.5 & $485 p^{2}\left({ }^{3} \mathrm{P}\right) 6 \mathrm{p}^{4} \mathrm{D}+195 \mathrm{p}^{2}\left({ }^{3} \mathrm{P}\right) 6 \mathrm{p}^{2} \mathrm{~S}+115 \mathrm{p}^{2}\left({ }^{3} \mathrm{P}\right) 6 \mathrm{p}^{2} \mathrm{P}$ \\
\hline 187532.9 & 187312 & 221 & 3.5 & $315 p^{2}\left({ }^{3} \mathrm{P}\right) 4 \mathrm{f}^{4} \mathrm{G}+275 \mathrm{p}^{2}\left({ }^{1} \mathrm{D}\right) 4 \mathrm{f}^{2} \mathrm{G}+235 \mathrm{p}^{2}\left({ }^{3} \mathrm{P}\right) 4 \mathrm{f}^{2} \mathrm{G}$ \\
\hline 188251.8 & 187942 & 310 & 4.5 & $835 p^{2}\left({ }^{3} P\right) 4 f^{4} G+95 p^{2}\left({ }^{3} P\right) 4 f^{4} F$ \\
\hline 188720.6 & 188478 & 243 & 2.5 & $525 p^{2}\left({ }^{3} P\right) 4 f^{2} D+195 p^{2}\left({ }^{3} P\right) 4 f^{4} G+115 p^{2}\left({ }^{3} P\right) 4 f^{4} D$ \\
\hline 189842.1 & 189879 & -37 & 3.5 & $545 p^{2}\left({ }^{3} P\right) 4 f^{4} D+185 p^{2}\left({ }^{3} P\right) 4 f^{2} G+155 p^{2}\left({ }^{1} D\right) 4 f^{2} G$ \\
\hline 190792.5 & 190927 & -135 & 1.5 & $385 p^{2}\left({ }^{3} P\right) 6 p^{4} D+145 p^{2}\left({ }^{3} P\right) 6 p^{4} P+125 p^{2}\left({ }^{3} P\right) 6 p^{2} D$ \\
\hline 191858.2 & 192042 & -184 & 1.5 & $435 \mathrm{p}^{2}\left({ }^{3} \mathrm{P}\right) 4 \mathrm{f}^{4} \mathrm{~F}+165 \mathrm{p}^{2}\left({ }^{3} \mathrm{P}\right) 4 \mathrm{f}^{4} \mathrm{D}+145 \mathrm{p}^{2}\left({ }^{3} \mathrm{P}\right) 4 \mathrm{f}^{2} \mathrm{D}$ \\
\hline 191978.1 & 192079 & -101 & 2.5 & $545 \mathrm{p}^{2}\left({ }^{3} \mathrm{P}\right) 4 \mathrm{f}^{4} \mathrm{D}+185 \mathrm{p}^{2}\left({ }^{3} \mathrm{P}\right) 4 \mathrm{f}^{2} \mathrm{D}+145 \mathrm{p}^{2}\left({ }^{3} \mathrm{P}\right) 4 \mathrm{f}^{4} \mathrm{~F}$ \\
\hline 193860.6 & 193915 & -54 & 0.5 & $475 p^{2}\left({ }^{3} P\right) 6 p^{2} S+365 p^{2}\left({ }^{3} P\right) 6 p^{4} D+135 p^{2}\left({ }^{3} P\right) 6 p{ }^{4} P$ \\
\hline 195784.6 & 195729 & 56 & 1.5 & $435 p^{2}\left({ }^{3} \mathrm{P}\right) 4 \mathrm{f}^{4} \mathrm{D}+175 \mathrm{p}^{2}\left({ }^{3} \mathrm{P}\right) 4 \mathrm{f}^{2} \mathrm{D}+95 \mathrm{p}^{2}\left({ }^{3} \mathrm{P}\right) 6 \mathrm{p}^{2} \mathrm{D}$ \\
\hline 196325.2 & 196734 & -409 & 3.5 & $565 \mathrm{p}^{2}\left({ }^{3} \mathrm{P}\right) 4 \mathrm{f}^{4} \mathrm{~F}+315 \mathrm{p}^{2}\left({ }^{1} \mathrm{D}\right) 4 \mathrm{f}^{2} \mathrm{~F}$ \\
\hline 196506.1 & 196718 & -212 & 2.5 & $515 p^{2}\left({ }^{3} \mathrm{P}\right) 4 \mathrm{f}^{4} \mathrm{~F}+225 \mathrm{p}^{2}\left({ }^{1} \mathrm{D}\right) 4 \mathrm{f}^{2} \mathrm{~F}+165 \mathrm{p}^{2}\left({ }^{3} \mathrm{P}\right) 4 \mathrm{f}^{4} \mathrm{D}$ \\
\hline 196654.7 & 196546 & 108 & 0.5 & $865 \mathrm{p}^{2}\left({ }^{3} \mathrm{P}\right) 4 \mathrm{f}^{4} \mathrm{D}+65 \mathrm{p}^{2}\left({ }^{1} \mathrm{D}\right) 4 \mathrm{f}^{2} \mathrm{P}$ \\
\hline 196724.9 & 196748 & -23 & 1.5 & $315 p^{2}\left({ }^{3} P\right) 6 p^{4} D+205 p^{2}\left({ }^{3} P\right) 6 p^{2} D+175 p^{2}\left({ }^{3} P\right) 4 f^{4} D$ \\
\hline 198943.1 & 199026 & -83 & 2.5 & $855 p^{2}\left({ }^{3} \mathrm{P}\right) 6 \mathrm{p}{ }^{4} \mathrm{D}+65 \mathrm{p}^{2}\left({ }^{1} \mathrm{D}\right) 6 \mathrm{p}^{2} \mathrm{~F}$ \\
\hline 199397.0 & 199389 & 8 & 1.5 & $335 \mathrm{p}^{2}\left({ }^{3} \mathrm{P}\right) 4 \mathrm{f}^{2} \mathrm{D}+215 \mathrm{p}^{2}\left({ }^{3} \mathrm{P}\right) 4 \mathrm{f}^{4} \mathrm{~F}+175 \mathrm{p}^{2}\left({ }^{3} \mathrm{P}\right) 6 \mathrm{p}^{4} \mathrm{~S}$ \\
\hline 200486.2 & 200403 & 83 & 2.5 & $335 p^{2}\left({ }^{3} \mathrm{P}\right) 6 \mathrm{p}^{4} \mathrm{P}+325 \mathrm{p}^{2}\left({ }^{3} \mathrm{P}\right) 6 \mathrm{p}^{2} \mathrm{D}+185 \mathrm{p}^{2}\left({ }^{1} \mathrm{D}\right) 6 \mathrm{p}^{2} \mathrm{D}$ \\
\hline 200899.4 & 200873 & 26 & 0.5 & $725 \mathrm{p}^{2}\left({ }^{3} \mathrm{P}\right) 6 \mathrm{p}{ }^{4} \mathrm{P}+115 \mathrm{p}^{2}\left({ }^{3} \mathrm{P}\right) 6 \mathrm{p}^{2} \mathrm{~S}+105 \mathrm{p}^{2}\left({ }^{3} \mathrm{P}\right) 6 \mathrm{p}{ }^{2} \mathrm{P}$ \\
\hline 201027.6 & 200782 & 245 & 1.5 & $295 \mathrm{p}^{2}\left({ }^{3} \mathrm{P}\right) 6 \mathrm{p}^{4} \mathrm{~S}+215 \mathrm{p}^{2}\left({ }^{3} \mathrm{P}\right) 6 \mathrm{p}^{2} \mathrm{D}+165 \mathrm{p}^{2}\left({ }^{3} \mathrm{P}\right) 4 \mathrm{f}^{2} \mathrm{D}$ \\
\hline 202076.1 & 202067 & 9 & 4.5 & $455 \mathrm{p}^{2}\left({ }^{3} \mathrm{P}\right) 4 \mathrm{f}^{4} \mathrm{~F}+345 \mathrm{p}^{2}\left({ }^{3} \mathrm{P}\right) 4 \mathrm{f}^{2} \mathrm{G}+115 \mathrm{p}^{2}\left({ }^{1} \mathrm{D}\right) 4 \mathrm{f}^{2} \mathrm{H}$ \\
\hline 202951.1 & 203327 & -376 & 3.5 & $585 \mathrm{p}^{2}\left({ }^{3} \mathrm{P}\right) 6 \mathrm{p}^{4} \mathrm{D}+245 \mathrm{p}^{2}\left({ }^{1} \mathrm{D}\right) 6 \mathrm{p}^{2} \mathrm{~F}+75 \mathrm{p}^{2}\left({ }^{3} \mathrm{P}\right) 4 \mathrm{f}^{2} \mathrm{~F}$ \\
\hline 204140.0 & 203905 & 235 & 1.5 & $475 p^{2}\left({ }^{3} \mathrm{P}\right) 6 \mathrm{p}^{4} \mathrm{P}+205 \mathrm{p}^{2}\left({ }^{3} \mathrm{P}\right) 6 \mathrm{p}^{4} \mathrm{~S}+155 \mathrm{p}^{2}\left({ }^{1} \mathrm{D}\right) 6 \mathrm{p}^{2} \mathrm{P}$ \\
\hline 205205.0 & 205427 & -222 & 2.5 & $465 \mathrm{p}^{2}\left({ }^{1} \mathrm{D}\right) 4 \mathrm{f}^{2} \mathrm{~F}+155 \mathrm{p}^{2}\left({ }^{3} \mathrm{P}\right) 4 \mathrm{f}^{2} \mathrm{~F}+85 \mathrm{p}^{2}\left({ }^{3} \mathrm{P}\right) 6 \mathrm{p}{ }^{4} \mathrm{P}$ \\
\hline 205216.7 & 204872 & 345 & 3.5 & $255 \mathrm{p}^{2}\left({ }^{3} \mathrm{P}\right) 4 \mathrm{f}^{2} \mathrm{~F}+195 \mathrm{p}^{2}\left({ }^{3} \mathrm{P}\right) 4 \mathrm{f}^{2} \mathrm{G}+145 \mathrm{p}^{2}\left({ }^{3} \mathrm{P}\right) 6 \mathrm{p}{ }^{4} \mathrm{D}$ \\
\hline 206061.2 & 205962 & 99 & 1.5 & $525 \mathrm{p}^{2}\left({ }^{3} \mathrm{P}\right) 6 \mathrm{p}^{2} \mathrm{P}+265 \mathrm{p}^{2}\left({ }^{1} \mathrm{D}\right) 6 \mathrm{p}^{2} \mathrm{D}+105 \mathrm{p}^{2}\left({ }^{1} \mathrm{D}\right) 6 \mathrm{p}^{2} \mathrm{P}$ \\
\hline 206216.2 & 206083 & 133 & 4.5 & $405 \mathrm{p}^{2}\left({ }^{1} \mathrm{D}\right) 4 \mathrm{f}^{2} \mathrm{G}+315 \mathrm{p}^{2}\left({ }^{1} \mathrm{D}\right) 4 \mathrm{f}^{2} \mathrm{H}+135 \mathrm{p}^{2}\left({ }^{3} \mathrm{P}\right) 4 \mathrm{f}^{4} \mathrm{~F}$ \\
\hline 206713.1 & 206868 & -155 & 3.5 & $305 \mathrm{p}^{2}\left({ }^{1} \mathrm{D}\right) 4 \mathrm{f}^{2} \mathrm{~F}+215 \mathrm{p}^{2}\left({ }^{1} \mathrm{D}\right) 4 \mathrm{f}^{2} \mathrm{G}+145 \mathrm{p}^{2}\left({ }^{3} \mathrm{P}\right) 4 \mathrm{f}^{4} \mathrm{~F}$ \\
\hline 207056.6 & 207071 & -14 & 2.5 & $175 p^{2}\left({ }^{3} \mathrm{P}\right) 6 \mathrm{p}{ }^{4} \mathrm{P}+165 \mathrm{p}^{2}\left({ }^{1} \mathrm{D}\right) 4 \mathrm{f}^{2} \mathrm{~F}+135 \mathrm{p}^{2}\left({ }^{3} \mathrm{P}\right) 6 \mathrm{p}{ }^{2} \mathrm{D}$ \\
\hline 208621.1 & 208870 & -249 & 2.5 & $435 p^{2}\left({ }^{3} \mathrm{P}\right) 4 \mathrm{f}^{2} \mathrm{~F}+155 \mathrm{p}^{2}\left({ }^{3} \mathrm{P}\right) 6 \mathrm{p}^{2} \mathrm{D}+95 \mathrm{p}^{2}\left({ }^{1} \mathrm{~S}\right) 4 \mathrm{f}^{2} \mathrm{~F}$ \\
\hline 209343.7 & 209185 & 158 & 0.5 & $685 p^{2}\left({ }^{3} \mathrm{P}\right) 6 \mathrm{p}^{2} \mathrm{P}+135 \mathrm{p}^{2}\left({ }^{3} \mathrm{P}\right) 6 \mathrm{p}^{2} \mathrm{~S}+75 \mathrm{p}^{2}\left({ }^{3} \mathrm{P}\right) 6 \mathrm{p}^{4} \mathrm{D}$ \\
\hline 213735.6 & 213529 & 207 & 1.5 & $765 \mathrm{p}^{2}\left({ }^{1} \mathrm{D}\right) 4 \mathrm{f}^{2} \mathrm{D}$ \\
\hline 215625.5 & 215579 & 47 & 2.5 & $395 p^{2}\left({ }^{1} \mathrm{D}\right) 6 \mathrm{p}^{2} \mathrm{~F}+245 \mathrm{p}^{2}\left({ }^{1} \mathrm{D}\right) 4 \mathrm{f}^{2} \mathrm{D}+165 \mathrm{p}^{2}\left({ }^{3} \mathrm{P}\right) 6 \mathrm{p}^{2} \mathrm{D}$ \\
\hline 216141.0 & 216086 & 55 & 1.5 & $355 \mathrm{p}^{2}\left({ }^{1} \mathrm{D}\right) 6 \mathrm{p}{ }^{2} \mathrm{D}+245 \mathrm{p}^{2}\left({ }^{1} \mathrm{D}\right) 6 \mathrm{p}{ }^{2} \mathrm{P}+75 \mathrm{p}^{2}\left({ }^{3} \mathrm{P}\right) 6 \mathrm{p}{ }^{4} \mathrm{P}$ \\
\hline 216910.7 & 216873 & 37 & 2.5 & $585 \mathrm{p}^{2}\left({ }^{1} \mathrm{D}\right) 6 \mathrm{p}^{2} \mathrm{D}+305 \mathrm{p}^{2}\left({ }^{3} \mathrm{P}\right) 6 \mathrm{p}{ }^{4} \mathrm{P}+65 \mathrm{p}^{2}\left({ }^{1} \mathrm{D}\right) 6 \mathrm{p}^{2} \mathrm{~F}$ \\
\hline 217239.7 & 217115 & 125 & 3.5 & $315 \mathrm{p}^{2}\left({ }^{1} \mathrm{D}\right) 6 \mathrm{p}^{2} \mathrm{~F}+305 \mathrm{p}^{2}\left({ }^{3} \mathrm{P}\right) 4 \mathrm{f}^{2} \mathrm{~F}+145 \mathrm{p}^{2}\left({ }^{3} \mathrm{P}\right) 6 \mathrm{p}{ }^{4} \mathrm{D}$ \\
\hline 219001.7 & 219675 & -673 & 2.5 & $365 \mathrm{p}^{2}\left({ }^{1} \mathrm{D}\right) 4 \mathrm{f}^{2} \mathrm{D}+175 \mathrm{p}^{2}\left({ }^{3} \mathrm{P}\right) 6 \mathrm{p}^{2} \mathrm{D}+165 \mathrm{p}^{2}\left({ }^{1} \mathrm{D}\right) 6 \mathrm{p}^{2} \mathrm{~F}$ \\
\hline 219717.3 & 219565 & 152 & 3.5 & $415 p^{2}\left({ }^{1} \mathrm{D}\right) 6 \mathrm{p}^{2} \mathrm{~F}+305 \mathrm{p}^{2}\left({ }^{3} \mathrm{P}\right) 4 \mathrm{f}^{2} \mathrm{~F}+125 \mathrm{p}^{2}\left({ }^{3} \mathrm{P}\right) 6 \mathrm{p}{ }^{4} \mathrm{D}$ \\
\hline 220081.6 & 220085 & -4 & 0.5 & $825 \mathrm{p}^{2}\left({ }^{1} \mathrm{D}\right) 6 \mathrm{p}^{2} \mathrm{P}+75 \mathrm{p}^{2}\left({ }^{3} \mathrm{P}\right) 6 \mathrm{p}^{2} \mathrm{~S}$ \\
\hline 220789.8 & 220565 & 225 & 0.5 & $825 \mathrm{p}^{2}\left({ }^{1} \mathrm{D}\right) 4 \mathrm{f}^{2} \mathrm{P}+55 \mathrm{p}^{2}\left({ }^{3} \mathrm{P}\right) 4 \mathrm{f}^{4} \mathrm{D}$ \\
\hline 224498.2 & 224669 & -171 & 1.5 & $355 \mathrm{p}^{2}\left({ }^{1} \mathrm{D}\right) 6 \mathrm{p}^{2} \mathrm{P}+265 \mathrm{p}^{2}\left({ }^{3} \mathrm{P}\right) 6 \mathrm{p}^{2} \mathrm{P}+145 \mathrm{p}^{2}\left({ }^{1} \mathrm{D}\right) 6 \mathrm{p}^{2} \mathrm{D}$ \\
\hline 228975.4 & 228900 & 75 & 3.5 & $795 \mathrm{p}^{2}\left({ }^{1} \mathrm{~S}\right) 4 \mathrm{f}^{2} \mathrm{~F}+85 \mathrm{p}^{2}\left({ }^{1} \mathrm{D}\right) 4 \mathrm{f}^{2} \mathrm{~F}$ \\
\hline 232811.4 & 232916 & -105 & 0.5 & $825 p^{2}\left({ }^{1} S\right) 6 p^{2} P+65 p^{2}\left({ }^{3} P\right) 6 p^{2} P$ \\
\hline 235560.7 & 235449 & 112 & 1.5 & $835 \mathrm{p}^{2}\left({ }^{1} \mathrm{~S}\right) 6 \mathrm{p}^{2} \mathrm{P}$ \\
\hline
\end{tabular}

Notes. Energies are given in $\mathrm{cm}^{-1} .{ }^{(a)}$ From Saloman (2004). ${ }^{(b)}$ This work. ${ }^{(c)}$ Only the first three components that are larger than $5 \%$ are given. 
T. Rauch et al.: Stellar laboratories. VIII.

Table B.3. continued.

\begin{tabular}{|c|c|c|c|c|}
\hline$E_{\exp }^{a}$ & $E_{\text {calc }}^{b}$ & $\Delta E$ & $J$ & Leading components (in \%) in $L S$ coupling $^{c}$ \\
\hline & & & & Even parity \\
\hline 99663.8 & 99384 & 279 & 2.5 & $845 \mathrm{~s} 5 \mathrm{p}^{4}{ }^{4} \mathrm{P}+95 \mathrm{p}^{2}\left({ }^{3} \mathrm{P}\right) 5 \mathrm{~d}^{4} \mathrm{P}$ \\
\hline 106923.2 & 106996 & -72 & 1.5 & $835 \mathrm{~s} 5 \mathrm{p}^{4}{ }^{4} \mathrm{P}+105 \mathrm{p}^{2}\left({ }^{3} \mathrm{P}\right) 5 \mathrm{~d}{ }^{4} \mathrm{P}$ \\
\hline 109254.4 & 109497 & -243 & 0.5 & $825 \mathrm{~s} 5 \mathrm{p}^{4}{ }^{4} \mathrm{P}+105 \mathrm{p}^{2}\left({ }^{3} \mathrm{P}\right) 5 \mathrm{~d}{ }^{4} \mathrm{P}+65 \mathrm{~s} 5 \mathrm{p}^{4}{ }^{2} \mathrm{~S}$ \\
\hline 121928.9 & 122134 & -205 & 1.5 & $565 \mathrm{~s} 5 \mathrm{p}^{4}{ }^{2} \mathrm{D}+155 \mathrm{p}^{2}\left({ }^{1} \mathrm{D}\right) 5 \mathrm{~d}^{2} \mathrm{D}+85 \mathrm{p}^{2}\left({ }^{3} \mathrm{P}\right) 5 \mathrm{~d}^{2} \mathrm{P}$ \\
\hline 125474.7 & 125429 & 46 & 2.5 & $695 \mathrm{~s} 5 \mathrm{p}^{4}{ }^{2} \mathrm{D}+195 \mathrm{p}^{2}\left({ }^{1} \mathrm{D}\right) 5 \mathrm{~d}^{2} \mathrm{D}+55 \mathrm{~s} 5 \mathrm{p}^{4}{ }^{4} \mathrm{P}$ \\
\hline 133027.4 & 132735 & 292 & 1.5 & $285 p^{2}\left({ }^{3} P\right) 5 d^{2} P+245 p^{2}\left({ }^{3} P\right) 5 d^{4} F+155 s 5 p^{4}{ }^{2} D$ \\
\hline 134980.6 & 135211 & -230 & 1.5 & $565 \mathrm{p}^{2}\left({ }^{3} \mathrm{P}\right) 5 \mathrm{~d}^{4} \mathrm{~F}+225 \mathrm{p}^{2}\left({ }^{3} \mathrm{P}\right) 5 \mathrm{~d}^{2} \mathrm{P}+135 \mathrm{~s} 5 \mathrm{p}^{4}{ }^{2} \mathrm{P}$ \\
\hline 136495.9 & 136607 & -111 & 2.5 & $635 p^{2}\left({ }^{3} P\right) 5 d^{4} F+225 p^{2}\left({ }^{3} P\right) 5 d^{4} D$ \\
\hline 136796.3 & 136825 & -29 & 0.5 & $425 \mathrm{p}^{2}\left({ }^{3} \mathrm{P}\right) 5 \mathrm{~d}^{2} \mathrm{P}+245 \mathrm{~s} 5 \mathrm{p}^{4}{ }^{2} \mathrm{P}+135 \mathrm{~s} 5 \mathrm{p}^{4}{ }^{2} \mathrm{~S}$ \\
\hline 141624.8 & 141917 & -292 & 3.5 & $785 p^{2}\left({ }^{3} P\right) 5 d^{4} F+165 p^{2}\left({ }^{3} P\right) 5 d^{4} D$ \\
\hline 141824.4 & 141665 & 159 & 2.5 & $395 \mathrm{p}^{2}\left({ }^{1} \mathrm{D}\right) 5 \mathrm{~d}^{2} \mathrm{~F}+315 \mathrm{p}^{2}\left({ }^{3} \mathrm{P}\right) 5 \mathrm{~d}^{2} \mathrm{~F}+185 \mathrm{p}^{2}\left({ }^{3} \mathrm{P}\right) 5 \mathrm{~d}^{4} \mathrm{~F}$ \\
\hline 145011.2 & 144856 & 155 & 3.5 & $345 \mathrm{p}^{2}\left({ }^{1} \mathrm{D}\right) 5 \mathrm{~d}^{2} \mathrm{~F}+325 \mathrm{p}^{2}\left({ }^{3} \mathrm{P}\right) 5 \mathrm{~d}^{4} \mathrm{D}+175 \mathrm{p}^{2}\left({ }^{3} \mathrm{P}\right) 5 \mathrm{~d}^{2} \mathrm{~F}$ \\
\hline 145105.7 & 145173 & -67 & 0.5 & $745 \mathrm{p}^{2}\left({ }^{3} \mathrm{P}\right) 5 \mathrm{~d}^{4} \mathrm{D}+135 \mathrm{~s} 5 \mathrm{p}^{4}{ }^{2} \mathrm{~S}$ \\
\hline 145991.1 & 146341 & -350 & 4.5 & $815 \mathrm{p}^{2}\left({ }^{3} \mathrm{P}\right) 5 \mathrm{~d}^{4} \mathrm{~F}+165 \mathrm{p}^{2}\left({ }^{1} \mathrm{D}\right) 5 \mathrm{~d}^{2} \mathrm{G}$ \\
\hline 146206.5 & 146263 & -57 & 1.5 & $785 \mathrm{p}^{2}\left({ }^{3} \mathrm{P}\right) 5 \mathrm{~d}^{4} \mathrm{D}+85 \mathrm{p}^{2}\left({ }^{3} \mathrm{P}\right) 5 \mathrm{~d}^{4} \mathrm{~F}$ \\
\hline 148685.0 & 148601 & 84 & 2.5 & $545 \mathrm{p}^{2}\left({ }^{3} \mathrm{P}\right) 5 \mathrm{~d}^{4} \mathrm{D}+125 \mathrm{p}^{2}\left({ }^{3} \mathrm{P}\right) 5 \mathrm{~d}^{4} \mathrm{~F}+125 \mathrm{p}^{2}\left({ }^{3} \mathrm{P}\right) 5 \mathrm{~d}^{2} \mathrm{~F}$ \\
\hline 150737.3 & 150659 & 78 & 0.5 & $415 s 5 p^{4}{ }^{2} S+225 p^{2}\left({ }^{3} P\right) 5 d^{2} P+145 p^{2}\left({ }^{1} D\right) 5 d^{2} S$ \\
\hline 155863.9 & 155893 & -29 & 3.5 & $425 p^{2}\left({ }^{3} P\right) 5 d^{4} D+205 p^{2}\left({ }^{3} P\right) 5 d^{2} F+185 p^{2}\left({ }^{1} D\right) 5 d^{2} G$ \\
\hline 157205.0 & 157289 & -84 & 0.5 & $695 \mathrm{p}^{2}\left({ }^{3} \mathrm{P}\right) 6 \mathrm{~s}{ }^{4} \mathrm{P}+185 \mathrm{p}^{2}\left({ }^{3} \mathrm{P}\right) 6 \mathrm{~s}^{2} \mathrm{P}+105 \mathrm{p}^{2}\left({ }^{1} \mathrm{~S}\right) 6 \mathrm{~s}^{2} \mathrm{~S}$ \\
\hline 159642.8 & 159380 & 263 & 2.5 & $715 p^{2}\left({ }^{3} P\right) 5 d^{4} P+95 p^{2}\left({ }^{3} P\right) 5 d^{4} D+65 s 5 p^{4}{ }^{4} P$ \\
\hline 160665.1 & 160696 & -31 & 3.5 & $665 \mathrm{p}^{2}\left({ }^{1} \mathrm{D}\right) 5 \mathrm{~d}^{2} \mathrm{G}+235 \mathrm{p}^{2}\left({ }^{1} \mathrm{D}\right) 5 \mathrm{~d}^{2} \mathrm{~F}+75 \mathrm{p}^{2}\left({ }^{3} \mathrm{P}\right) 5 \mathrm{~d}^{4} \mathrm{D}$ \\
\hline 161434.7 & 161471 & -36 & 1.5 & $595 \mathrm{p}^{2}\left({ }^{3} \mathrm{P}\right) 5 \mathrm{~d}^{4} \mathrm{P}+155 \mathrm{p}^{2}\left({ }^{1} \mathrm{D}\right) 5 \mathrm{~d}^{2} \mathrm{P}+75 \mathrm{~s} 5 \mathrm{p}^{4}{ }^{4} \mathrm{P}$ \\
\hline 162866.5 & 162752 & 115 & 0.5 & $635 \mathrm{p}^{2}\left({ }^{3} \mathrm{P}\right) 5 \mathrm{~d}^{4} \mathrm{P}+135 \mathrm{p}^{2}\left({ }^{1} \mathrm{D}\right) 5 \mathrm{~d}^{2} \mathrm{P}+75 \mathrm{~s} 5 \mathrm{p}^{4}{ }^{4} \mathrm{P}$ \\
\hline 163463.1 & 163608 & -145 & 4.5 & $815 p^{2}\left({ }^{1} D\right) 5 d^{2} G+165 p^{2}\left({ }^{3} P\right) 5 d^{4} F$ \\
\hline 163596.7 & 163137 & 459 & 1.5 & $325 \mathrm{~s} 5 \mathrm{p}^{4}{ }^{2} \mathrm{P}+305 \mathrm{p}^{2}\left({ }^{3} \mathrm{P}\right) 5 \mathrm{~d}^{2} \mathrm{D}+165 \mathrm{p}^{2}\left({ }^{1} \mathrm{~S}\right) 5 \mathrm{~d}^{2} \mathrm{D}$ \\
\hline 165280.0 & 165268 & 12 & 1.5 & $185 \mathrm{p}^{2}\left({ }^{1} \mathrm{D}\right) 5 \mathrm{~d}^{2} \mathrm{P}+175 \mathrm{~s} 5 \mathrm{p}^{4}{ }^{2} \mathrm{P}+175 \mathrm{p}^{2}\left({ }^{3} \mathrm{P}\right) 5 \mathrm{~d}^{2} \mathrm{D}$ \\
\hline 165995.3 & 166060 & -65 & 1.5 & $805 \mathrm{p}^{2}\left({ }^{3} \mathrm{P}\right) 6 \mathrm{~s}{ }^{4} \mathrm{P}+75 \mathrm{p}^{2}\left({ }^{3} \mathrm{P}\right) 6 \mathrm{~s}{ }^{2} \mathrm{P}$ \\
\hline 167206.4 & 167606 & -399 & 0.5 & $655 \mathrm{p}^{2}\left({ }^{3} \mathrm{P}\right) 6 \mathrm{~s}^{2} \mathrm{P}+235 \mathrm{p}^{2}\left({ }^{3} \mathrm{P}\right) 6 \mathrm{~s}^{4} \mathrm{P}$ \\
\hline 169001.5 & 168667 & 335 & 2.5 & $545 \mathrm{p}^{2}\left({ }^{3} \mathrm{P}\right) 5 \mathrm{~d}^{2} \mathrm{D}+155 \mathrm{p}^{2}\left({ }^{3} \mathrm{P}\right) 5 \mathrm{~d}^{2} \mathrm{~F}+115 \mathrm{p}^{2}\left({ }^{1} \mathrm{D}\right) 5 \mathrm{~d}^{2} \mathrm{~F}$ \\
\hline 170490.3 & 170444 & 47 & 2.5 & $615 \mathrm{p}^{2}\left({ }^{3} \mathrm{P}\right) 6 \mathrm{~s}{ }^{4} \mathrm{P}+295 \mathrm{p}^{2}\left({ }^{1} \mathrm{D}\right) 6 \mathrm{~s}^{2} \mathrm{D}$ \\
\hline 172892.2 & 172288 & 604 & 0.5 & $495 \mathrm{p}^{2}\left({ }^{1} \mathrm{D}\right) 5 \mathrm{~d}^{2} \mathrm{P}+245 \mathrm{~s} 5 \mathrm{p}^{4}{ }^{2} \mathrm{P}+115 \mathrm{p}^{2}\left({ }^{3} \mathrm{P}\right) 5 \mathrm{~d}^{4} \mathrm{P}$ \\
\hline 173221.8 & 172467 & 755 & 1.5 & $545 \mathrm{p}^{2}\left({ }^{3} \mathrm{P}\right) 6 \mathrm{~s}{ }^{2} \mathrm{P}+335 \mathrm{p}^{2}\left({ }^{1} \mathrm{D}\right) 6 \mathrm{~s}^{2} \mathrm{D}$ \\
\hline 176041.9 & 175731 & 311 & 2.5 & $275 \mathrm{p}^{2}\left({ }^{1} \mathrm{D}\right) 5 \mathrm{~d}^{2} \mathrm{~F}+205 \mathrm{p}^{2}\left({ }^{3} \mathrm{P}\right) 5 \mathrm{~d}^{2} \mathrm{~F}+165 \mathrm{p}^{2}\left({ }^{1} \mathrm{D}\right) 5 \mathrm{~d}^{2} \mathrm{D}$ \\
\hline 176122.2 & 176020 & 102 & 1.5 & $555 \mathrm{p}^{2}\left({ }^{1} \mathrm{D}\right) 5 \mathrm{~d}^{2} \mathrm{D}+145 \mathrm{~s} 5 \mathrm{p}^{4}{ }^{2} \mathrm{D}+125 \mathrm{p}^{2}\left({ }^{1} \mathrm{D}\right) 5 \mathrm{~d}^{2} \mathrm{P}$ \\
\hline 177923.3 & 177771 & 153 & 3.5 & $575 \mathrm{p}^{2}\left({ }^{3} \mathrm{P}\right) 5 \mathrm{~d}^{2} \mathrm{~F}+305 \mathrm{p}^{2}\left({ }^{1} \mathrm{D}\right) 5 \mathrm{~d}^{2} \mathrm{~F}+65 \mathrm{p}^{2}\left({ }^{1} \mathrm{D}\right) 5 \mathrm{~d}^{2} \mathrm{G}$ \\
\hline 177951.1 & 178819 & -868 & 0.5 & $265 \mathrm{p}^{2}\left({ }^{1} \mathrm{D}\right) 5 \mathrm{~d}^{2} \mathrm{P}+245 \mathrm{p}^{2}\left({ }^{1} \mathrm{D}\right) 5 \mathrm{~d}^{2} \mathrm{~S}+195 \mathrm{~s} 5 \mathrm{p}^{4}{ }^{2} \mathrm{P}$ \\
\hline 179000.5 & 178344 & 657 & 2.5 & $355 \mathrm{p}^{2}\left({ }^{1} \mathrm{D}\right) 5 \mathrm{~d}^{2} \mathrm{D}+295 \mathrm{p}^{2}\left({ }^{1} \mathrm{~S}\right) 5 \mathrm{~d}^{2} \mathrm{D}+105 \mathrm{p}^{2}\left({ }^{3} \mathrm{P}\right) 5 \mathrm{~d}^{2} \mathrm{D}$ \\
\hline 182571.0 & 184149 & -1578 & 1.5 & $335 \mathrm{p}^{2}\left({ }^{1} \mathrm{D}\right) 6 \mathrm{~s}^{2} \mathrm{D}+215 \mathrm{p}^{2}\left({ }^{1} \mathrm{D}\right) 5 \mathrm{~d}^{2} \mathrm{P}+135 \mathrm{p}^{2}\left({ }^{3} \mathrm{P}\right) 5 \mathrm{~d}^{2} \mathrm{P}$ \\
\hline 186048.6 & 185632 & 417 & 2.5 & $655 \mathrm{p}^{2}\left({ }^{1} \mathrm{D}\right) 6 \mathrm{~s}^{2} \mathrm{D}+255 \mathrm{p}^{2}\left({ }^{3} \mathrm{P}\right) 6 \mathrm{~s}{ }^{4} \mathrm{P}$ \\
\hline 187546.9 & 187664 & -117 & 1.5 & $295 \mathrm{p}^{2}\left({ }^{1} \mathrm{D}\right) 6 \mathrm{~s}^{2} \mathrm{D}+275 \mathrm{p}^{2}\left({ }^{3} \mathrm{P}\right) 6 \mathrm{~s}^{2} \mathrm{P}+185 \mathrm{p}^{2}\left({ }^{1} \mathrm{D}\right) 5 \mathrm{~d}^{2} \mathrm{P}$ \\
\hline 188272.6 & 188073 & 200 & 0.5 & $365 \mathrm{p}^{2}\left({ }^{1} \mathrm{D}\right) 5 \mathrm{~d}^{2} \mathrm{~S}+185 \mathrm{~s} 5 \mathrm{p}^{4}{ }^{2} \mathrm{~S}+185 \mathrm{~s} 5 \mathrm{p}^{4}{ }^{2} \mathrm{P}$ \\
\hline 190030.5 & 190017 & 14 & 2.5 & $425 \mathrm{p}^{2}\left({ }^{1} \mathrm{~S}\right) 5 \mathrm{~d}^{2} \mathrm{D}+235 \mathrm{p}^{2}\left({ }^{3} \mathrm{P}\right) 5 \mathrm{~d}^{2} \mathrm{D}+125 \mathrm{p}^{2}\left({ }^{3} \mathrm{P}\right) 5 \mathrm{~d}^{2} \mathrm{~F}$ \\
\hline 190369.3 & 190894 & -525 & 1.5 & $595 \mathrm{p}^{2}\left({ }^{1} \mathrm{~S}\right) 5 \mathrm{~d}^{2} \mathrm{D}+325 \mathrm{p}^{2}\left({ }^{3} \mathrm{P}\right) 5 \mathrm{~d}^{2} \mathrm{D}$ \\
\hline 202054.6 & 202052 & 3 & 0.5 & $865 \mathrm{p}^{2}\left({ }^{1} \mathrm{~S}\right) 6 \mathrm{~s}{ }^{2} \mathrm{~S}+65 \mathrm{p}^{2}\left({ }^{3} \mathrm{P}\right) 6 \mathrm{~s}{ }^{4} \mathrm{P}$ \\
\hline
\end{tabular}


Table B.4. Comparison between available experimental and calculated energy levels in Xe V.

\begin{tabular}{|c|c|c|c|c|}
\hline$E_{\exp }{ }^{a}$ & $E_{\text {calc }^{b}}^{b}$ & $\Delta E$ & $J$ & Leading components (in \%) in $L S$ coupling $^{c}$ \\
\hline \multicolumn{5}{|r|}{ Even parity } \\
\hline 0.0 & -6 & 6 & 0 & $875 p^{23} \mathrm{P}+105 \mathrm{p}^{2}{ }^{1} \mathrm{~S}$ \\
\hline 9291.8 & 9290 & 2 & 1 & $975 \mathrm{p}^{23} \mathrm{P}$ \\
\hline 14126.7 & 14142 & -15 & 2 & $665 p^{23} \mathrm{P}+315 \mathrm{p}^{2}{ }^{1} \mathrm{D}$ \\
\hline 28411.2 & 28402 & 9 & 2 & $655 p^{2}{ }^{1} \mathrm{D}+315 \mathrm{p}^{2} \mathrm{P}$ \\
\hline 44470.4 & 44471 & -1 & 0 & $865 p^{2}{ }^{1} S+105 p^{2}{ }^{3} P$ \\
\hline 186746.7 & 186635 & 111 & 3 & $545 \mathrm{p} 4 \mathrm{f}^{3} \mathrm{G}+375 \mathrm{p} 4 \mathrm{f}^{1} \mathrm{~F}$ \\
\hline 189663.8 & 189859 & -196 & 3 & $445 \mathrm{p} 4 \mathrm{f}^{3} \mathrm{~F}+215 \mathrm{p} 4 \mathrm{f}^{3} \mathrm{D}+215 \mathrm{p} 4 \mathrm{f}^{1} \mathrm{~F}$ \\
\hline 190644.7 & 190745 & -101 & 4 & $525 \mathrm{p} 4 \mathrm{f}^{3} \mathrm{G}+365 \mathrm{p} 4 \mathrm{f}^{3} \mathrm{~F}+75 \mathrm{p} 4 \mathrm{f}^{1} \mathrm{G}$ \\
\hline 191603.5 & 191400 & 204 & 2 & $785 \mathrm{p} 4 \mathrm{f}^{3} \mathrm{~F}+115 \mathrm{p} 4 \mathrm{f}^{3} \mathrm{D}+75 \mathrm{p} 4 \mathrm{f}^{1} \mathrm{D}$ \\
\hline 200010.2 & 199897 & 113 & 3 & $325 \mathrm{p} 4 \mathrm{f}^{1} \mathrm{~F}+325 \mathrm{p} 4 \mathrm{f}^{3} \mathrm{G}+315 \mathrm{p} 4 \mathrm{f}^{3} \mathrm{~F}$ \\
\hline 201545.2 & 201629 & -84 & 4 & $565 \mathrm{p} 4 \mathrm{f}^{3} \mathrm{~F}+395 \mathrm{p} 4 \mathrm{f}^{3} \mathrm{G}$ \\
\hline 202281.8 & 202341 & -59 & 5 & $955 \mathrm{p} 4 \mathrm{f}^{3} \mathrm{G}$ \\
\hline 205758.8 & 205942 & -183 & 3 & $715 p 4 f^{3} D+185 p 4 f^{3} F+75 p 4 f^{1} F$ \\
\hline 207366.7 & 207261 & 106 & 2 & $765 \mathrm{p} 4 \mathrm{f}^{3} \mathrm{D}+155 \mathrm{p} 4 \mathrm{f}^{3} \mathrm{~F}$ \\
\hline 209310.7 & 209194 & 116 & 1 & $955 \mathrm{p} 4 \mathrm{f}^{3} \mathrm{D}$ \\
\hline 214317.7 & 214380 & -62 & 4 & $875 \mathrm{p} 4 \mathrm{f}^{1} \mathrm{G}$ \\
\hline 216745.6 & 216701 & 44 & 2 & $825 \mathrm{p} 4 \mathrm{f}^{1} \mathrm{D}+85 \mathrm{p} 4 \mathrm{f}^{3} \mathrm{D}$ \\
\hline 228064.9 & 228416 & -351 & 1 & $625 p 6 p^{3} D+315 p 6 p{ }^{1} P$ \\
\hline 233999.3 & 233744 & 255 & 0 & $835 p 6 p^{3} P+105 p 6 p^{1} S$ \\
\hline 234455.6 & 234336 & 120 & 1 & $415 p 6 p^{3} P+185 p 6 p{ }^{1} P+175 p 6 p^{3} D$ \\
\hline 235178.9 & 235165 & 14 & 2 & $715 p 6 p^{3} D+115 p 6 p{ }^{1} D+115 p 6 p^{3} P$ \\
\hline 243216.5 & 243049 & 168 & 1 & $425 p 6 p^{3} P+245 p 6 p{ }^{1} \mathrm{P}+125 p 6 p^{3} D$ \\
\hline 244821.3 & 244637 & 184 & 2 & $535 p 6 p^{3} P+225 p 6 p^{3} D+165 p 6 p^{1} D$ \\
\hline 246208.0 & 245966 & 242 & 3 & $955 \mathrm{p}^{2} \mathrm{p}^{3} \mathrm{D}$ \\
\hline 247810.4 & 247929 & -119 & 1 & $405 p 6{ }^{3} S+365 s 5 p^{2} 5 d^{5} D+65 p 6 p^{1} P$ \\
\hline 250557.2 & 251379 & -822 & 2 & $635 p 6 p^{1} D+185 p 6 p^{3} P+65 p 4{ }^{1} D$ \\
\hline \multicolumn{5}{|r|}{ Odd parity } \\
\hline 92182.8 & 92194 & -11 & 2 & $935 s 5 p^{35} s+65 s 5 p^{3}{ }^{3} P$ \\
\hline 115286.3 & 115441 & -155 & 1 & $745 s 5 p^{3}{ }^{3} D+105 s 5 p^{3}{ }^{3} p+95 p 5 d^{3} D$ \\
\hline 116097.0 & 116138 & -41 & 2 & $725 s 5 p^{3}{ }^{3} D+135 s 5 p^{3}{ }^{3} P+85 p 5 d^{3} D$ \\
\hline 119919.0 & 119913 & 6 & 3 & $885 \mathrm{~s} 5 \mathrm{p}^{3}{ }^{3} \mathrm{D}+95 \mathrm{p} 5 \mathrm{~d}^{3} \mathrm{D}$ \\
\hline 133408.1 & 133488 & -80 & 0 & $905 \mathrm{~s} 5 \mathrm{p}^{3}{ }^{3} \mathrm{P}+85 \mathrm{p} 5 \mathrm{~d}^{3} \mathrm{P}$ \\
\hline 134575.2 & 134507 & 68 & 1 & $755 s 5 p^{3}{ }^{3} P+105 s 5 p^{3}{ }^{3} D+75 p 5 d^{3} P$ \\
\hline 134702.7 & 134496 & 206 & 2 & $415 \mathrm{~s} 5 \mathrm{p}^{3}{ }^{3} \mathrm{P}+205 \mathrm{~s} 5 \mathrm{p}^{3}{ }^{1} \mathrm{D}+125 \mathrm{~s} 5 \mathrm{p}^{3}{ }^{3} \mathrm{D}$ \\
\hline 145807.0 & 145525 & 282 & 2 & $315 \mathrm{p} 5 \mathrm{~d}^{1} \mathrm{D}+285 \mathrm{~s} 5 \mathrm{p}^{3}{ }^{1} \mathrm{D}+275 \mathrm{~s} 5 \mathrm{p}^{3}{ }^{3} \mathrm{P}$ \\
\hline 155518.1 & 155393 & 125 & 1 & $665 s 5 p^{3} 3+245 s 5 p^{3}{ }^{1} P$ \\
\hline 156506.8 & 156303 & 204 & 2 & $865 \mathrm{p} 5 \mathrm{~d}^{3} \mathrm{~F}+85 \mathrm{~s} 5 \mathrm{p}^{3}{ }^{1} \mathrm{D}$ \\
\hline 160630.4 & 160677 & -47 & 3 & $895 \mathrm{p} 5 \mathrm{~d}^{3} \mathrm{~F}$ \\
\hline 169672.6 & 170261 & -588 & 1 & $325 s 5 p^{3}{ }^{1} P+205 p 5 d^{1} P+145 s 5 p^{3}{ }^{3} S$ \\
\hline 169799.4 & 170055 & -255 & 4 & $965 \mathrm{p} 5 \mathrm{~d}^{3} \mathrm{~F}$ \\
\hline 170987.6 & 170919 & 69 & 2 & $455 \mathrm{p} 5 \mathrm{~d}^{3} \mathrm{P}+235 \mathrm{p} 5 \mathrm{~d}^{3} \mathrm{D}+125 \mathrm{~s} 5 \mathrm{p}^{3}{ }^{1} \mathrm{D}$ \\
\hline 173071.7 & 173063 & 9 & 1 & $505 p 5 d^{3} D+235 s 5 p^{3} 1+115 s 5 p^{3}{ }^{3} s$ \\
\hline 181004.3 & 181097 & -93 & 2 & $395 \mathrm{p} 5 \mathrm{~d}^{3} \mathrm{D}+305 \mathrm{p} 5 \mathrm{~d}^{1} \mathrm{D}+185 \mathrm{~s} 5 \mathrm{p}^{3}{ }^{1} \mathrm{D}$ \\
\hline 182167.2 & 182145 & 22 & 3 & $755 p 5 d^{3} D+75 s 5 p^{3}{ }^{3} D+65 p 5 d^{1} F$ \\
\hline 183025.2 & 182962 & 63 & 0 & $875 \mathrm{p} 5 \mathrm{~d}^{3} \mathrm{P}+85 \mathrm{~s} 5 \mathrm{p}^{3}{ }^{3} \mathrm{P}$ \\
\hline 184147.6 & 184100 & 48 & 1 & $645 p 5 d^{3} P+185 p 5 d^{3} D+75 s 5 p^{3}{ }^{3} P$ \\
\hline 185795.0 & 185780 & 15 & 2 & $415 p 5 d^{3} P+225 p 5 d^{3} D+145 p 5 d{ }^{1} D$ \\
\hline 194033.1 & 194105 & -72 & 0 & $965 \mathrm{p} 6 \mathrm{~s}^{3} \mathrm{P}$ \\
\hline 194138.0 & 194159 & -21 & 3 & $865 \mathrm{p} 5 \mathrm{~d}^{1} \mathrm{~F}+85 \mathrm{p} 5 \mathrm{~d}^{3} \mathrm{D}$ \\
\hline
\end{tabular}

Notes. Energies are given in $\mathrm{cm}^{-1} .{ }^{(a)}$ From Saloman (2004) and Raineri et al. (2009). ${ }^{(b)}$ This work. ${ }^{(c)}$ Only the first three components that are larger than $5 \%$ are given. 
T. Rauch et al.: Stellar laboratories. VIII.

Table B.4. continued.

\begin{tabular}{|c|c|c|c|c|}
\hline$E_{\mathrm{exp}}{ }^{a}$ & $E_{\text {calc }}^{b}$ & $\Delta E$ & $J$ & Leading components (in \%) in $L S$ coupling ${ }^{c}$ \\
\hline 194232.9 & 194190 & 43 & 1 & $545 p 6 s{ }^{3} \mathrm{P}+255 \mathrm{p} 6 \mathrm{~s}{ }^{1} \mathrm{P}+125 \mathrm{p} 5 \mathrm{~d}^{1} \mathrm{P}$ \\
\hline 199959.0 & 199730 & 229 & 1 & $555 \mathrm{p} 5 \mathrm{~d}^{1} \mathrm{P}+245 \mathrm{p} 6 \mathrm{~s}^{3} \mathrm{P}+95 \mathrm{~s} 5 \mathrm{p}^{3}{ }^{1} \mathrm{P}$ \\
\hline 209068.9 & 209078 & -9 & 2 & $965 \mathrm{p} 6 \mathrm{~s}{ }^{3} \mathrm{P}$ \\
\hline 213040.2 & 213053 & -13 & 1 & $715 \mathrm{p} 6 \mathrm{~s}{ }^{1} \mathrm{P}+185 \mathrm{p} 6 \mathrm{~s}{ }^{3} \mathrm{P}+65 \mathrm{p} 5 \mathrm{~d}{ }^{1} \mathrm{P}$ \\
\hline 287391.0 & 287420 & -29 & 2 & $355 p 6 d^{3} P+295 p 6 d^{3} D+185 p 6 d^{1} D$ \\
\hline 287696.0 & 288003 & -307 & 3 & $365 p 6 d^{3} F+315 s 5 p^{2} 4 f^{3} G+105 p 6 d^{3} D$ \\
\hline 288830.0 & 288586 & 244 & 1 & $505 p 6 d^{3} D+145 p 6 d{ }^{3} P+135 p 6 d{ }^{1} P$ \\
\hline 298053.0 & 298054 & -1 & 1 & $695 \mathrm{p} 7 \mathrm{~s}{ }^{3} \mathrm{P}+295 \mathrm{p} 7 \mathrm{~s}{ }^{1} \mathrm{P}$ \\
\hline 298739.0 & 298717 & 22 & 4 & $915 \mathrm{p} 6 \mathrm{~d}^{3} \mathrm{~F}$ \\
\hline 299596.0 & 299417 & 179 & 2 & $535 p 6 d^{1} D+175 p 6 d{ }^{3} D+155 p 6 d^{3} F$ \\
\hline 300327.0 & 300484 & -157 & 3 & $605 p 6 d^{3} D+265 p 6 d^{3} F$ \\
\hline 301555.0 & 301796 & -241 & 1 & $655 p 6 d^{3} P+245 p 6 d^{3} D$ \\
\hline 301998.0 & 301794 & 204 & 0 & $925 \mathrm{p} 6 \mathrm{~d}^{3} \mathrm{P}$ \\
\hline 306065.0 & 306081 & -16 & 1 & $745 p 6 d^{1} P+115 p 6 d^{3} D+75 p 6{ }^{3} P$ \\
\hline 312956.0 & 312959 & -3 & 2 & $995 \mathrm{p} 7 \mathrm{~s}{ }^{3} \mathrm{P}$ \\
\hline 313883.0 & 313880 & 3 & 1 & $705 \mathrm{p} 7 \mathrm{~s}{ }^{1} \mathrm{P}+295 \mathrm{p} 7 \mathrm{~s}{ }^{3} \mathrm{P}$ \\
\hline
\end{tabular}

